\title{
Quiver Varieties and Branching ${ }^{\star}$
}

\author{
Hiraku NAKAJIMA †‡ \\ ${ }^{\dagger}$ Department of Mathematics, Kyoto University, Kyoto 606-8502, Japan \\ $\ddagger$ Research Institute for Mathematical Sciences, Kyoto University, Kyoto 606-8502, Japan \\ E-mail: nakajima@math.kyoto-u.ac.jp
}

Received September 15, 2008, in final form January 05, 2009; Published online January 11, 2009 doi:10.3842/SIGMA.2009.003

\begin{abstract}
Braverman and Finkelberg recently proposed the geometric Satake correspondence for the affine Kac-Moody group $G_{\text {aff }}$ [Braverman A., Finkelberg M., arXiv:0711.2083]. They conjecture that intersection cohomology sheaves on the Uhlenbeck compactification of the framed moduli space of $G_{\mathrm{cpt}}$-instantons on $\mathbb{R}^{4} / \mathbb{Z}_{r}$ correspond to weight spaces of representations of the Langlands dual group $G_{\text {aff }}^{\vee}$ at level $r$. When $G=\operatorname{SL}(l)$, the Uhlenbeck compactification is the quiver variety of type $\mathfrak{s l}(r)_{\text {aff }}$, and their conjecture follows from the author's earlier result and I. Frenkel's level-rank duality. They further introduce a convolution diagram which conjecturally gives the tensor product multiplicity [Braverman A., Finkelberg M., Private communication, 2008]. In this paper, we develop the theory for the branching in quiver varieties and check this conjecture for $G=\operatorname{SL}(l)$.
\end{abstract}

Key words: quiver variety; geometric Satake correspondence; affine Lie algebra; intersection cohomology

2000 Mathematics Subject Classification: 17B65; 14D21

\section{Contents}

1 Introduction $\quad 2$

2 Partial resolutions $\quad 4$

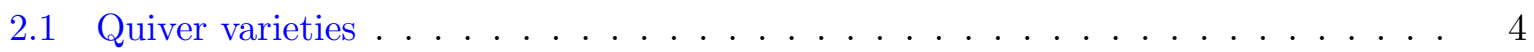

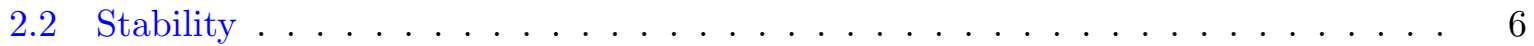

2.3 Face structure on the set of stability conditions . . . . . . . . . . 8

2.4 Nonemptiness of $\mathfrak{M}_{\zeta}^{\mathrm{s}} \ldots \ldots \ldots \ldots \ldots$

2.5 Partial resolutions $\ldots \ldots \ldots \ldots \ldots \ldots \ldots \ldots$

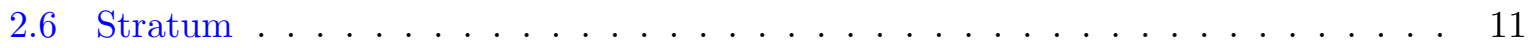

2.7 Local structure . . . . . . . . . . . . . . . . . . . . . . . . 12

2.8 Example: Levi factors of parabolic subalgebras . . . . . . . . . . . . . . . 14

3 Instantons on ALE spaces $\quad 16$

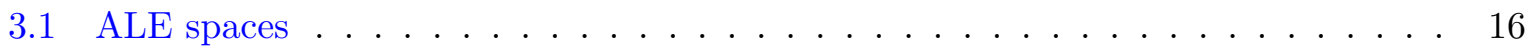

3.2 Sheaves and instantons on ALE spaces . . . . . . . . . . . . . . 18

4 Crystal and the branching $\quad 21$

5 Convolution algebra and partial resolution $\quad 22$

5.1 General results . . . . . . . . . . . . . . . . . . . . . 22

5.2 The restriction to a Levi factor . . . . . . . . . . . . . . . . . . . . . 24

${ }^{\star}$ This paper is a contribution to the Special Issue on Kac-Moody Algebras and Applications. The full collection is available at http://www.emis.de/journals/SIGMA/Kac-Moody_algebras.html 
5.3 Restriction to the affine Lie algebra of a Levi factor . . . . . . . . . . . . . 26

6 MV cycles for the double affine Grassmannian of type $A \quad 29$

A Level-rank duality $\quad \mathbf{3 2}$

A.1 Weight multiplicities . . . . . . . . . . . . . . . . 32

A.2 Tensor product multiplicities $\ldots \ldots \ldots \ldots$

References

36

\section{Introduction}

In $[22,24]$ the author showed that the top degree homology group of a Lagrangian subvariety $\mathfrak{L}$ in a quiver variety $\mathfrak{M}$ has a structure of an integrable highest weight representation of a KacMoody Lie algebra $\mathfrak{g}$. In a subsequent work [26] the author showed that the equivariant $K$ homology group of $\mathfrak{L}$ has a structure of an $\ell$-integrable highest weight representation of the quantum loop algebra $\mathbf{U}_{q}(\mathbf{L} \mathfrak{g})$ (e.g., the quantum affine algebra if $\mathfrak{g}$ is of finite type, the quantum toroidal algebra if $\mathfrak{g}$ is of affine type). As an application, the characters of arbitrary irreducible representations of $\mathbf{U}_{q}(\mathbf{L} \mathfrak{g})$ were computed in terms of the intersection cohomology (IC for short) groups associated with graded/cyclic quiver varieties (= the fixed point set in the quiver variety with respect to $\mathbb{C}^{*}$ /cyclic group action), and hence analogs of Kazhdan-Lusztig polynomials. This result cannot be proved by a purely algebraic method. See [28] for a survey.

The quiver variety $\mathfrak{M}$ is defined as a geometric invariant theory quotient of an affine variety $\mu^{-1}(0)$ by a product of general linear groups with respect to a particular choice of a stability condition $\zeta$, a lift of an action to the trivial line bundle over $\mu^{-1}(0)$. Let us denote $\mathfrak{M}$ by $\mathfrak{M}_{\zeta}$ hereafter to emphasize a choice of a stability condition. We can consider other stability conditions. For example, if we choose the trivial lift $\zeta=0$, then we get an affine algebraic variety $\mathfrak{M}_{0}$. It has been studied already in the literature, and played important roles. For example, we have a projective morphism $\pi_{0, \zeta}: \mathfrak{M}_{\zeta} \rightarrow \mathfrak{M}_{0}$, and $\mathfrak{L}$ is the inverse image $\pi_{0, \zeta}^{-1}(0)$ of a distinguished point $0 \in \mathfrak{M}_{0}$. Moreover $\mathfrak{M}_{0}$ has a natural stratification parametrized by conjugacy classes of stabilizers, and their IC complexes give the restriction multiplicities of the above $\mathbf{U}_{q}(\mathbf{L} \mathfrak{g})$-module to $\mathbf{U}_{q}(\mathfrak{g})$.

In this paper we study a more general stability condition $\zeta$ whose corresponding variety $\mathfrak{M}_{\zeta} \bullet$ sits between $\mathfrak{M}_{\zeta}$ and $\mathfrak{M}_{0}$ : the morphism $\pi$ factorizes $\mathfrak{M}_{\zeta} \stackrel{\pi_{\zeta} \bullet \zeta}{\longrightarrow} \mathfrak{M}_{\zeta} \bullet \stackrel{\pi_{0, \zeta}}{\longrightarrow} \mathfrak{M}_{0}$. Under a mild assumption, $\mathfrak{M}_{\zeta}$ is a partial resolution of singularities of $\mathfrak{M}_{0}$, while $\mathfrak{M}_{\zeta}$ is a full resolution. Then we show that the top degree cohomology group of $\pi_{0, \zeta}^{-1} \bullet(0)$ with coefficients in the IC complex of a stratum of $\mathfrak{M}_{\zeta} \bullet$ gives the restriction multiplicities of an integrable highest weight representation to a subalgebra determined by $\zeta$. This follows from a general theory for representations constructed by the convolution product [3], but we identify the subalgebras with the Levi subalgebra of $\mathfrak{g}$ or the affine Lie algebra of the Levi subalgebra for certain choices of $\zeta \bullet$ (see Theorems 5.6, 5.15). (For a quiver variety of finite or affine type, they exhaust all choices up to the Weyl group action.)

This work is motivated by a recent proposal by Braverman and Finkelberg $[4,5]$ on a conjectural affine Kac-Moody group analog of the geometric Satake correspondence. (See also a recent paper [6] for the affine analog of the classical Satake correspondence.) The ordinary geometric Satake correspondence says that the category of equivariant perverse sheaves on the affine Grassmannian $G(\mathcal{K}) / G(\mathcal{O})$ associated with a finite dimensional complex simple Lie group $G$ is equivalent to the category of finite dimensional representations of the Langlands dual group $G^{\vee}$, so that IC sheaves of $G(\mathcal{O})$-orbits correspond to irreducible representations. Here $\mathcal{K}=\mathbb{C}((s))$, $\mathcal{O}=\mathbb{C}[[s]]$. If we would have the affine Grassmannian for the affine Kac-Moody group $G_{\text {aff }}$ 
(hence called the double affine Grassmannian), then it should correspond to representations of the affine Kac-Moody group $G_{\text {aff }}^{\vee}$. It is not clear whether we can consider IC sheaves on the double affine Grassmannian $G_{\text {aff }}(\mathcal{K}) / G_{\text {aff }}(\mathcal{O})$ or not, but Braverman and Finkelberg [4, 5] propose that the transversal slice of a $G_{\text {aff }}(\mathcal{O})$-orbit in the closure of a bigger $G_{\text {aff }}(\mathcal{O})$-orbit should be the Uhlenbeck partial compactification of the framed moduli space of $G_{\mathrm{cpt}}$-instantons ${ }^{1}$ on $\mathbb{R}^{4} / \mathbb{Z}_{r}$ (more precisely on $S^{4} / \mathbb{Z}_{r}=\mathbb{R}^{4} \cup\{\infty\} / \mathbb{Z}_{r}$ ), where $r$ is the level of the corresponding representation of $G_{\mathrm{aff}}^{\vee}$. Here $G_{\mathrm{cpt}}$ is the maximal compact subgroup of $G$.

When $G=\operatorname{SL}(l)$, the Uhlenbeck partial compactification in question is the quiver variety $\mathfrak{M}_{0}$ of the affine type $A_{r-1}^{(1)}=\mathfrak{s l}(r)_{\text {aff }}$. As mentioned above, the representation of $\mathfrak{s l}(r)_{\text {aff }}$ can be constructed from $\mathfrak{M}_{0}$ in the framework of quiver varieties. The level of the representation is $l$. Now the proposed conjectural link to the representation theory of $G_{\text {aff }}^{\vee}=\operatorname{PGL}(l)_{\text {aff }}$ is provided by the theory of quiver varieties composed with I. Frenkel's level rank duality [9] between representations of $\mathfrak{s l}(r)$ aff with level $l$ and of $\mathfrak{s l}(l)$ aff with level $r$ :

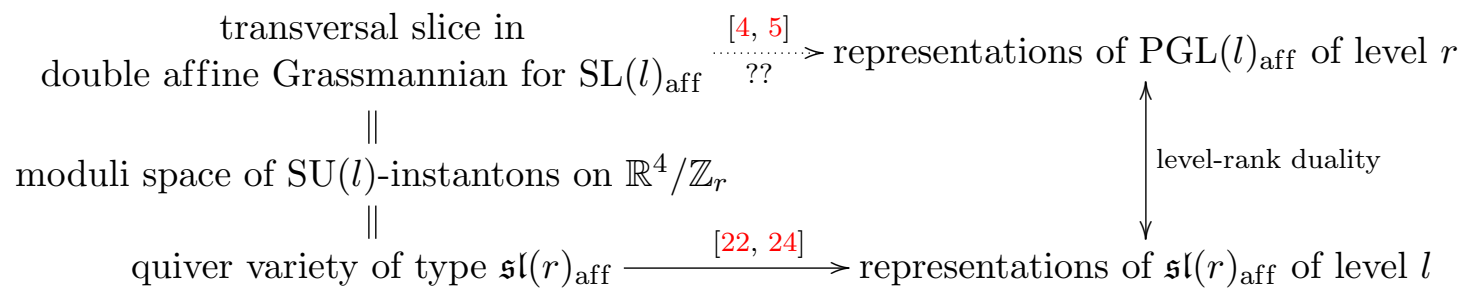

In fact, the existence of this commutative diagram is one of the sources of Braverman and Finkelberg's proposal, and has been already used to check that the intersection cohomology group of the Uhlenbeck compactification has dimension equal to the corresponding weight space [4].

One of the most important ingredients in the geometric Satake correspondence is the convolution diagram which gives the tensor product of representations. Braverman and Finkelberg [5] propose that its affine analog is the Uhlenbeck compactification of the framed moduli space of

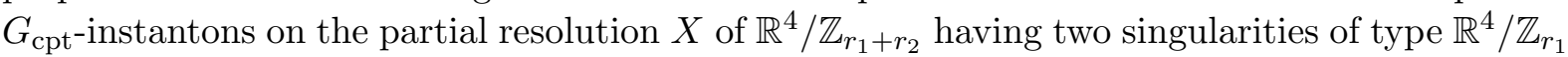
and $\mathbb{R}^{4} / \mathbb{Z}_{r_{2}}$ connected by $\mathbb{P}^{1}$. Now again for $G=\mathrm{SL}(l)$, the Uhlenbeck compactification is the quiver variety $\mathfrak{M}_{\zeta}$ (see Section 3 ). Since the tensor product of a level $r_{1}$ representation and a level $r_{2}$ representation corresponds to the restriction to $\left(\mathfrak{s l}\left(r_{1}\right) \oplus \mathfrak{s l}\left(r_{2}\right)\right)_{\text {aff }}$ under the level-rank duality, their proposal can be checked from the theory developed in this paper.

Let us explain several other things treated/not treated in this paper. Recall that Kashiwara and Saito $[13,33]$ gave a structure of the crystal on the set of irreducible components of $\mathfrak{L}$, which is isomorphic to the Kashiwara's crystal of the corresponding representation of $\mathbf{U}_{q}(\mathfrak{g})$. In Section 4 we study the branching in the crystal when $\mathfrak{M}_{\zeta} \bullet$ corresponds to a Levi subalgebra $\mathfrak{g}_{I^{0}}$ of $\mathfrak{g}$ corresponding to a subset $I^{0}$ of the index set $I$ of simple roots. From a general theory on the crystal, the $\mathfrak{g}_{I^{0}}$-crystal of the restriction of a $\mathbf{U}_{q}(\mathfrak{g})$-representation to the subalgebra $\mathbf{U}_{q}\left(\mathfrak{g}_{I^{0}}\right) \subset \mathbf{U}_{q}(\mathfrak{g})$ is given by forgetting $i$-arrows with $i \notin I^{0}$. Each connected component contains an element corresponding to a highest weight vector. An irreducible component of $\mathfrak{L}$ is a highest weight vector in the $\mathfrak{g}_{I^{0}}$-crystal if and only if it is mapped birationally onto its image under $\pi_{\zeta \cdot \zeta}$ (see Theorem 4.1).

One can also express the branching coefficients of the restriction from $\mathbf{U}_{q}(\mathbf{L} \mathfrak{g})$ to $\mathbf{U}_{q}\left(\mathbf{L}\left(\mathfrak{g}_{I_{0}}\right)\right)$ in terms of IC sheaves on graded/cyclic quiver varieties, but we omit the statements, as a reader can write down them rather obviously if he/she knows [26] and understands Theorem 5.6.

In Section 6 we check several statements concerning the affine analogs of Mirković-Vilonen cycles for $G=\mathrm{SL}(r)$, proposed by Braverman-Finkelberg [5].

\footnotetext{
${ }^{1}$ By the Hitchin-Kobayashi correspondence, proved in [1] in this setting, the framed moduli space of $G_{\mathrm{cpt}}{ }^{-}$ instantons on $S^{4} / \mathbb{Z}_{r}$ is isomorphic to the framed moduli space of holomorphic $G$-bundles on $\mathbb{P}^{2} / \mathbb{Z}_{r}=\left(\mathbb{C}^{2} \cup \ell_{\infty}\right) / \mathbb{Z}_{r}$.
} 
In Appendix A we review the level-rank duality following [11, 31]. The results are probably well-known to experts, but we need to check how the degree operators are interchanged.

After the first version of the paper was submitted, one of the referees pointed out that the restriction to a Levi subalgebra of $\mathfrak{g}$ was already considered by Malkin $[19, \S 3]$ at least in the level of the crystal. Thus the result in Section 4 is not new. But the author decided to keep Section 4 , as it naturally arises as a good example of the theory developed in this paper.

\section{Partial resolutions}

\subsection{Quiver varieties}

Suppose that a finite graph is given. Let $I$ be the set of vertices and $E$ the set of edges. Let $\mathbf{C}=\left(c_{i j}\right)$ be the Cartan matrix of the graph, namely

$$
c_{i j}= \begin{cases}2-2(\text { the number of edges joining } i \text { to itself }) & \text { if } i=j, \\ -(\text { the number of edges joining } i \text { to } j) & \text { if } i \neq j .\end{cases}
$$

If the graph does not contain edge loops, it is a symmetric generalized Cartan matrix, and we have the corresponding symmetric Kac-Moody algebra $\mathfrak{g}$. We also define $a_{i j}=2 \delta_{i j}-c_{i j}$. Then $\mathbf{A}=\left(a_{i j}\right)$ is the adjacency matrix when there are no edge loops, but not in general.

In $[22,24]$ the author assumed that the graph does not contain edge loops (i.e., no edges joining a vertex with itself), but most of results (in particular definitions, natural morphisms, etc) hold without this assumption. And more importantly we need to consider such cases in local models even if we study quiver varieties without edge loops. See Section 2.7. So we do not assume the condition.

Let $H$ be the set of pairs consisting of an edge together with its orientation. So we have $\# H=2 \# E$. For $h \in H$, we denote by $\mathrm{i}(h)$ (resp. o $(h))$ the incoming (resp. outgoing) vertex of $h$. For $h \in H$ we denote by $\bar{h}$ the same edge as $h$ with the reverse orientation. Choose and fix an orientation $\Omega$ of the graph, i.e., a subset $\Omega \subset H$ such that $\bar{\Omega} \cup \Omega=H, \Omega \cap \bar{\Omega}=\varnothing$. The pair $(I, \Omega)$ is called a quiver.

Let $V=\left(V_{i}\right)_{i \in I}$ be a finite dimensional $I$-graded vector space over $\mathbb{C}$. The dimension of $V$ is a vector

$$
\operatorname{dim} V=\left(\operatorname{dim} V_{i}\right)_{i \in I} \in \mathbb{Z}_{\geq 0}^{I} .
$$

We denote the $i^{\text {th }}$ coordinate vector by $\mathbf{e}_{i}$.

If $V^{1}$ and $V^{2}$ are $I$-graded vector spaces, we define vector spaces by

$$
\mathrm{L}\left(V^{1}, V^{2}\right) \stackrel{\text { def }}{=} \bigoplus_{i \in I} \operatorname{Hom}\left(V_{i}^{1}, V_{i}^{2}\right), \quad \mathrm{E}\left(V^{1}, V^{2}\right) \stackrel{\text { def }}{=} \bigoplus_{h \in H} \operatorname{Hom}\left(V_{\mathrm{o}(h)}^{1}, V_{\mathrm{i}(h)}^{2}\right) .
$$

For $B=\left(B_{h}\right) \in \mathrm{E}\left(V^{1}, V^{2}\right)$ and $C=\left(C_{h}\right) \in \mathrm{E}\left(V^{2}, V^{3}\right)$, let us define a multiplication of $B$ and $C$ by

$$
C B \stackrel{\text { def }}{=}\left(\sum_{\mathrm{i}(h)=i} C_{h} B_{\bar{h}}\right)_{i} \in \mathrm{L}\left(V^{1}, V^{3}\right) .
$$

Multiplications $b a, B a$ of $a \in \mathrm{L}\left(V^{1}, V^{2}\right), b \in \mathrm{L}\left(V^{2}, V^{3}\right), B \in \mathrm{E}\left(V^{2}, V^{3}\right)$ are defined in the obvious manner. If $a \in \mathrm{L}\left(V^{1}, V^{1}\right)$, its trace $\operatorname{tr}(a)$ is understood as $\sum_{i} \operatorname{tr}\left(a_{i}\right)$.

For two $I$-graded vector spaces $V, W$ with $\mathbf{v}=\operatorname{dim} V, \mathbf{w}=\operatorname{dim} W$, we consider the vector space given by

$$
\mathbf{M} \equiv \mathbf{M}(\mathbf{v}, \mathbf{w}) \equiv \mathbf{M}(V, W) \stackrel{\text { def }}{=} \mathrm{E}(V, V) \oplus \mathrm{L}(W, V) \oplus \mathrm{L}(V, W),
$$


where we use the notation $\mathbf{M}(\mathbf{v}, \mathbf{w})$ when the isomorphism classes of $I$-graded vector spaces $V, W$ are concerned, and $\mathbf{M}$ when $V, W$ are clear in the context. The dimension of $\mathbf{M}$ is ${ }^{t} \mathbf{v}(2 \mathbf{w}+(2 \mathbf{I}-\mathbf{C}) \mathbf{v})$, where $\mathbf{I}$ is the identity matrix. The above three components for an element of $\mathbf{M}$ will be denoted by $B=\bigoplus B_{h}, a=\bigoplus a_{i}, b=\bigoplus b_{i}$ respectively.

When the graph has no edge loop and corresponds to the symmetric Kac-Moody Lie algebra $\mathfrak{g}$, we consider $\mathbf{v}, \mathbf{w}$ as weights of $\mathfrak{g}$ by the following rule: $\mathbf{v}=\sum_{i} v_{i} \alpha_{i}, \mathbf{w}=\sum_{i} w_{i} \Lambda_{i}$, where $\alpha_{i}$ and $\Lambda_{i}$ are a simple root and a fundamental weight respectively.

The orientation $\Omega$ defines a function $\varepsilon: H \rightarrow\{ \pm 1\}$ by $\varepsilon(h)=1$ if $h \in \Omega, \varepsilon(h)=-1$ if $h \in \bar{\Omega}$. We consider $\varepsilon$ as an element of $\mathrm{L}(V, V)$. Let us define a symplectic form $\omega$ on $\mathbf{M}$ by

$$
\omega\left((B, a, b),\left(B^{\prime}, a^{\prime}, b^{\prime}\right)\right) \stackrel{\text { def }}{=} \operatorname{tr}\left(\varepsilon B B^{\prime}\right)+\operatorname{tr}\left(a b^{\prime}-a^{\prime} b\right) .
$$

Let $G \equiv G_{\mathbf{v}} \equiv G_{V}$ be an algebraic group defined by

$$
G \equiv G_{\mathbf{v}} \equiv G_{V} \stackrel{\text { def }}{=} \prod_{i} \mathrm{GL}\left(V_{i}\right),
$$

where we use the notation $G_{\mathbf{v}}$ (resp. $G_{V}$ ) when we want to emphasize the dimension (resp. the vector space). Its Lie algebra is the direct sum $\bigoplus_{i} \mathfrak{g l}\left(V_{i}\right)$. The group $G$ acts on $\mathbf{M}$ by

$$
(B, a, b) \mapsto g \cdot(B, a, b) \stackrel{\text { def }}{=}\left(g B g^{-1}, g a, b g^{-1}\right)
$$

preserving the symplectic structure. The space $\mathbf{M}$ has a factor

$$
\mathbf{M}^{\mathrm{el}} \stackrel{\text { def }}{=} \bigoplus_{h: \mathrm{o}(h)=\mathrm{i}(h)} \mathbb{C i d}_{V_{\mathrm{o}(h)}}
$$

on which $G$ acts trivially. This has a 2-dimensional space for each edge loop, and hence has dimension $\sum_{i}\left(2-c_{i i}\right)$ in total.

The moment map vanishing at the origin is given by

$$
\mu(B, a, b)=\varepsilon B B+a b \in \mathrm{L}(V, V),
$$

where the dual of the Lie algebra of $G$ is identified with $\mathrm{L}(V, V)$ via the trace.

We call a point $(B, a, b)$ in $\mu^{-1}(0)$ (or more generally $\mathbf{M}(V, W)$ ) a module. In fact, it is really a module of a certain path algebra (with relations) after Crawley-Boevey's trick in $[8$, the end of Introduction] (see also Section 2.4), but this view point is not necessary, and a reader could consider this is simply naming.

We would like to consider a 'symplectic quotient' of $\mu^{-1}(0)$ divided by $G$. However we cannot expect the set-theoretical quotient to have a good property. Therefore we consider the quotient using the geometric invariant theory. Then the quotient depends on an additional parameter $\zeta=\left(\zeta_{i}\right)_{i \in I} \in \mathbb{Z}^{I}$ as follows: Let us define a character of $G$ by

$$
\chi_{\zeta}(g) \stackrel{\text { def }}{=} \prod_{i \in I}\left(\operatorname{det} g_{i}\right)^{-\zeta_{i}} .
$$

Let $A\left(\mu^{-1}(0)\right)$ be the coordinate ring of the affine variety $\mu^{-1}(0)$. Set

$$
A\left(\mu^{-1}(0)\right) \text { G, } \chi_{\zeta}^{n} \stackrel{\text { def }}{=}\left\{f \in A\left(\mu^{-1}(0)\right) \mid f(g \cdot(B, a, b))=\chi_{\zeta}(g)^{n} f((B, a, b))\right\} .
$$

The direct sum with respect to $n \in \mathbb{Z}_{\geq 0}$ is a graded algebra, hence we can define

$$
\mathfrak{M}_{\zeta} \equiv \mathfrak{M}_{\zeta}(\mathbf{v}, \mathbf{w}) \equiv \mathfrak{M}_{\zeta}(V, W) \stackrel{\text { def }}{=} \operatorname{Proj}\left(\bigoplus_{n \geq 0} A\left(\mu^{-1}(0)\right)^{G, \chi_{\zeta}^{n}}\right)
$$


This is the quiver variety introduced in [22]. Since this space is unchanged when we replace $\chi$ by a positive power $\chi^{N}(N>0)$, this space is well-defined for $\zeta \in \mathbb{Q}^{I}$. We call $\zeta$ a stability parameter.

When $W=0$, the scalar subgroup $\mathbb{C}^{*}$ id acts trivially on $\mathbf{M}$, so we choose the parameter $\zeta$ so that $\sum_{i} \zeta_{i} \operatorname{dim} V_{i}=0$, and take the 'quotient' with respect to the group $P G \stackrel{\text { def }}{=} G / \mathbb{C}^{*}$ id and the character $\chi: P G \rightarrow \mathbb{C}^{*}$.

Since $G$ acts trivially on the factor (2.1), we have the factorization

$$
\mathfrak{M}_{\zeta}=\mathbf{M}^{\mathrm{el}} \times \mathfrak{M}_{\zeta}^{\mathrm{norm}}
$$

where $\mathfrak{M}_{\zeta}^{\text {norm }}$ is the symplectic quotient of the space of datum $(B, a, b)$ satisfying $\operatorname{tr}\left(B_{h}\right)=0$ for any $h$ with $\mathrm{i}(h)=\mathrm{o}(h)$.

\section{$2.2 \quad$ Stability}

We will describe $\mathfrak{M}_{\zeta}$ as a moduli space. We also introduce Harder-Narasimhan and JordanHölder filtrations, for which we need to add an additional variable $\zeta_{\infty} \in \mathbb{Q}$ to the stability parameter $\zeta \in \mathbb{Q}^{I}$. We write $\tilde{\zeta}=\left(\zeta, \zeta_{\infty}\right) \in \mathbb{Q}^{I \sqcup\{\infty\}}$. Let

$$
\begin{aligned}
& \tilde{\zeta}(V, W) \stackrel{\text { def }}{=} \sum_{i \in I} \zeta_{i} \operatorname{dim} V_{i}+\zeta_{\infty}\left(1-\delta_{W 0}\right), \\
& \theta_{\tilde{\zeta}}(V, W) \stackrel{\text { def }}{=} \frac{\tilde{\zeta}(V, W)}{1-\delta_{W 0}+\sum_{i \in I} \operatorname{dim} V_{i}},
\end{aligned}
$$

where $\delta_{W 0}$ is 1 if $W=0$ and 0 otherwise, and we implicitly assume $V \neq 0$ or $W \neq 0$ in the definition of $\theta_{\tilde{\zeta}}(V, W)$.

Definition 2.4. (1) Suppose a module $(B, a, b) \in \mathbf{M}(V, W)$ is given. We consider an $I$-graded subspace $V^{\prime}$ in $V$ such that either of the following two conditions is satisfied:

(a) $V^{\prime}$ is contained in $\operatorname{Ker} b$ and $B$-invariant,

(b) $V^{\prime}$ contains $\operatorname{Im} a$ and is $B$-invariant.

In the first case we define the submodule $\left.(B, a, b)\right|_{\left(V^{\prime}, 0\right)} \in \mathbf{M}\left(V^{\prime}, 0\right)$ by putting $\left.B\right|_{V^{\prime}}$ on $\mathrm{E}\left(V^{\prime}, V^{\prime}\right)$ (and 0 on $\left.\mathrm{L}\left(0, V^{\prime}\right) \oplus \mathrm{L}\left(V^{\prime}, 0\right)=0\right)$. We define the quotient module $\left.(B, a, b)\right|_{\left(V / V^{\prime}, W\right)} \in \mathbf{M}\left(V / V^{\prime}, W\right)$ by putting the homomorphisms induced from $B, a, b$ on $\mathrm{E}\left(V / V^{\prime}, V / V^{\prime}\right), \mathrm{L}\left(W, V / V^{\prime}\right), \mathrm{L}\left(V / V^{\prime}, W\right)$ respectively. In the second case we define the submodule $\left.(B, a, b)\right|_{\left(V^{\prime}, W\right)} \in \mathbf{M}\left(V^{\prime}, W\right)$ and the quotient module $\left.(B, a, b)\right|_{\left(V / V^{\prime}, 0\right)} \in \mathbf{M}\left(V / V^{\prime}, 0\right)$ in a similar way. We may also say $\left(V^{\prime}, 0\right)$ (resp. $\left.\left(V^{\prime}, W\right)\right),\left(V / V^{\prime}, W\right)$ (resp. $\left.\left(V / V^{\prime}, 0\right)\right)$ a submodule and a quotient module of $(B, a, b)$ in the case (a) (resp. (b)). When we want to treat the two cases simultaneously we write a submodule $\left(V^{\prime}, \delta W\right)$ or a quotient module $(V, W) /\left(V^{\prime}, \delta W\right)$, where we mean $\delta W$ is either 0 or $W$.

(2) A module $(B, a, b) \in \mathbf{M}(V, W)$ is $\tilde{\zeta}$-semistable if we have

$$
\theta_{\tilde{\zeta}}\left(V^{\prime}, \delta W\right) \leq \theta_{\tilde{\zeta}}(V, W)
$$

for any nonzero submodule $\left(V^{\prime}, \delta W\right)$ of $(B, a, b)$.

We say $(B, a, b)$ is $\tilde{\zeta}$-stable if the strict inequalities hold unless $\left(V^{\prime}, \delta W\right)=(V, W)$.

We say $(B, a, b)$ is $\tilde{\zeta}$-polystable if it is a direct sum of $\tilde{\zeta}$-stable modules having the same $\theta_{\tilde{\zeta}}$-value.

The function $\theta_{\tilde{\zeta}}$ is an analog of the slope of a torsion free sheaf appearing in the definition of its stability. We have the following property analogous to one for the slope. 
Lemma 2.6. Let $\left(V^{\prime}, \delta W\right)$ be a submodule of $(B, a, b)$ and $(V, W) /\left(V^{\prime}, \delta W\right)$ be the quotient. Then

$$
\theta_{\tilde{\zeta}}\left(V^{\prime}, \delta W\right) \leq(\operatorname{resp} . \geq,=) \theta_{\tilde{\zeta}}(V, W) \Longleftrightarrow \theta_{\tilde{\zeta}}\left((V, W) /\left(V^{\prime}, \delta W\right)\right) \geq(\operatorname{resp} . \leq,=) \theta_{\tilde{\zeta}}(V, W) .
$$

The $\tilde{\zeta}$-(semi)stability condition is unchanged even if we shift the stability parameter $\tilde{\zeta}$ by a vector $c(1,1, \ldots, 1) \in \mathbb{Q}^{I \sqcup\{\infty\}}$ with a constant $c \in \mathbb{Q}$. Therefore we may normalize so that $\theta_{\tilde{\zeta}}(V, W)=0$ by choosing $c=-\theta_{\tilde{\zeta}}(V, W)$. We then take the component $\zeta \in \mathbb{Q}^{I}$ after this normalization and then define $\mathfrak{M}_{\zeta}$ as in the previous subsection. Moreover if $W=0$, the additional component $\zeta_{\infty}$ is clearly irrelevant, and the normalization condition $\theta_{\tilde{\zeta}}(V, W)=0$ just means $\sum_{i} \zeta_{i} \operatorname{dim} V_{i}=0$. Therefore we can also apply the construction in the previous subsection.

Conversely when $\zeta \in \mathbb{Q}^{I}$ and $I$-graded vector spaces $V, W$ are given as in the previous subsection, we define $\tilde{\zeta}$ by the following convention: If $W \neq 0$, take $\zeta_{\infty}$ so that $\theta_{\tilde{\zeta}}(V, W)=0$. If $W=0$, then we have assumed $\sum \zeta_{i} \operatorname{dim} V_{i}=0$. So we just put $\zeta_{\infty}=0$. Once this convention becomes clear, we say $\zeta$-(semi)stable instead of $\tilde{\zeta}$-(semi)stable.

Example 2.7. (1) Under this convention and the assumption $\zeta_{i}>0$ for all $i \in I$, the inequality $(2.5)$ is never satisfied for a nonzero submodule of the form $\left(V^{\prime}, 0\right)$. Also (2.5) is always satisfied for a submodule $\left(V^{\prime}, W\right)$. Therefore the $\zeta$-stability is equivalent to the nonexistence of nonzero $B$-invariant $I$-graded subspaces $V^{\prime}=\bigoplus V_{i}^{\prime}$ contained in Ker $b$ (and in this case $\zeta$-stability and $\zeta$-semistability are equivalent). This is the stability condition used in [24, 3.9].

(2) Let $\zeta_{i}=0$ for all $i$. Then any module is $\zeta$-semistable. A module is $\zeta$-stable if and only if it is simple, i.e., has no nontrivial submodules.

We recall Harder-Narasimhan and Jordan-Hölder filtrations. We need to fix $\tilde{\zeta} \in \mathbb{Q}^{I \sqcup\{\infty\}}$ and do not take the normalization condition $\theta_{\tilde{\zeta}}=0$ as we want to compare $\theta_{\tilde{\zeta}}$-values for various dimension vectors.

Theorem 2.8 ([32]). (1) A module $(B, a, b)$ has the unique Harder-Narasimhan filtration: a flag of I-graded subspaces

$$
V=V^{0} \supset V^{1} \supset \cdots \supset V^{N} \supset V^{N+1}=0
$$

and an integer $0 \leq k_{W} \leq N$ such that

(a) $\left.(B, a, b)\right|_{\left(V^{k+1}, \delta_{k+1} W\right)}$ is a submodule of $\left.(B, a, b)\right|_{\left(V^{k}, \delta_{k} W\right)}$ for $0 \leq k \leq N$, where $\delta_{k} W=W$ for $0 \leq k \leq k_{W}$ and 0 for $k_{W}+1 \leq k \leq N+1$,

(b) the quotient module $\left.\operatorname{gr}_{k}(B, a, b) \stackrel{\text { def }}{=}(B, a, b)\right|_{\left(V^{k}, \delta_{k} W\right) /\left(V^{k+1}, \delta_{k+1} W\right)}$ is $\tilde{\zeta}$-semistable for $0 \leq$ $k \leq N$ and

$$
\theta_{\tilde{\zeta}}\left(\operatorname{gr}_{0}(B, a, b)\right)<\theta_{\tilde{\zeta}}\left(\operatorname{gr}_{1}(B, a, b)\right)<\cdots<\theta_{\tilde{\zeta}}\left(\operatorname{gr}_{N}(B, a, b)\right)
$$

(2) A $\tilde{\zeta}$-semistable module $(B, a, b)$ has a Jordan-Hölder filtration: a flag of I-graded subspaces

$$
V=V^{0} \supset V^{1} \supset \cdots \supset V^{N} \supset V^{N+1}=0
$$

and an integer $0 \leq k_{W} \leq N$ such that

(a) the same as (a) in (1), 
(b) the quotient module $\left.\operatorname{gr}_{k}(B, a, b) \stackrel{\text { def }}{=}(B, a, b)\right|_{\left(V^{k}, \delta_{k} W\right) /\left(V^{k+1}, \delta_{k+1} W\right)}$ is $\tilde{\zeta}$-stable for $0 \leq k \leq N$ and

$$
\theta_{\tilde{\zeta}}\left(\operatorname{gr}_{0}(B, a, b)\right)=\theta_{\tilde{\zeta}}\left(\operatorname{gr}_{1}(B, a, b)\right)=\cdots=\theta_{\tilde{\zeta}}\left(\operatorname{gr}_{N}(B, a, b)\right) .
$$

Moreover the isomorphism class of $\bigoplus \operatorname{gr}_{k}(B, a, b)$ is uniquely determined by that of $(B, a, b)$.

Let $H_{\zeta}^{\text {s }}\left(\right.$ resp. $\left.H_{\zeta}^{\text {ss }}\right)$ be the set of $\zeta$-stable (resp. $\zeta$-semistable) modules in $\mu^{-1}(0) \subset \mathbf{M}$.

We say two $\zeta$-semistable modules $(B, a, b),\left(B^{\prime}, a^{\prime}, b^{\prime}\right)$ are $S$-equivalent when the closures of orbits intersect in $H_{\zeta}^{\mathrm{ss}}$.

Proposition 2.9. (1) ([14]) $\mathfrak{M}_{\zeta}$ is a coarse moduli space of $\zeta$-semistable modules modulo $S$ equivalences. Moreover, there is an open subset $\mathfrak{M}_{\zeta}^{\mathrm{s}} \subset \mathfrak{M}_{\zeta}$, which is a fine moduli space of $\zeta$-stable modules modulo isomorphisms.

(2) ([14]) Two ל-semistable modules are S-equivalent if and only if their Jordan-Hölder filtration in Theorem 2.8(2) have the same composition factors. Thus $\mathfrak{M}_{\zeta}$ is a coarse moduli space of $\zeta$-polystable modules modulo isomorphisms.

(3) (See $[22,2.6]$ or $[24,3.12])$ Suppose $\mathbf{w} \neq 0$. Then $\mathfrak{M}_{\zeta}^{\mathrm{s}}$, provided it is nonempty, is smooth of dimension ${ }^{t} \mathbf{v}(2 \mathbf{w}-\mathbf{C v})$. If $\mathbf{w}=0$, then $\mathfrak{M}_{\zeta}^{\mathrm{s}}$, provided it is nonempty, is smooth of dimension $2-{ }^{t} \mathbf{v C v}$.

The $S$-equivalence class of a point $(B, a, b) \in \mu^{-1}(0) \cap H_{\zeta}^{\text {ss }}$ will be denoted by $[B, a, b]$, and is considered as a closed point in $\mathfrak{M}_{\zeta}$.

We recall the following result $[22,3.1,4.2]$ :

Theorem 2.10. Suppose $\mathfrak{M}_{\zeta}^{\mathrm{s}}=\mathfrak{M}_{\zeta}$. Then $\mathfrak{M}_{\zeta}$ is diffeomorphic to a nonsingular affine algebraic variety.

\subsection{Face structure on the set of stability conditions}

Fix a dimension vector $\mathbf{v}=\left(v_{i}\right)$. Let

$$
\begin{aligned}
& R_{+} \stackrel{\text { def }}{=}\left\{\theta=\left(\theta_{i}\right)_{i \in I} \in \mathbb{Z}_{\geq 0}^{I} \mid{ }^{t} \theta \mathbf{C} \theta \leq 2\right\} \backslash\{0\}, \\
& R_{+}(\mathbf{v}) \stackrel{\text { def }}{=}\left\{\theta \in R_{+} \mid \theta_{i} \leq v_{i} \quad \text { for all } i \in I\right\}, \\
& D_{\theta} \stackrel{\text { def }}{=}\left\{\zeta=\left(\zeta_{i}\right) \in \mathbb{Q}^{I} \mid \zeta \cdot \theta=0\right\} \quad \text { for } \theta \in R_{+},
\end{aligned}
$$

where $\zeta \cdot \theta=\sum_{i} \zeta_{i} \theta_{i}$. When the graph is of Dynkin or affine type, $R_{+}$is the set of positive roots, and $D_{\theta}$ is the wall defined by the root $\theta$ ([12, Proposition 5.10]). In general, $R_{+}$may be an infinite set, but $R_{+}(\mathbf{v})$ is always finite.

Lemma 2.11 (cf. $[22,2.8]$ ). Suppose $\mathfrak{M}_{\zeta}^{\mathrm{s}}(\mathbf{v}, 0) \neq \varnothing$. Then $\mathbf{v} \in R_{+}$.

This is clear from the dimension formula Proposition $2.9(3)$ as $\operatorname{dim} \mathfrak{M}_{\zeta}^{\mathrm{s}}(\mathbf{v}, 0)$ must be nonnegative.

The set $R_{+}(\mathbf{v})$ defines a system of faces. A subset $F \subset \mathbb{R}^{I}$ is a face if there exists a disjoint decomposition $R_{+}(\mathbf{v})=R_{+}^{0}(\mathbf{v}) \sqcup R_{+}^{+}(\mathbf{v}) \sqcup R_{+}^{-}(\mathbf{v})$ such that

$$
\left.F=\left\{\zeta \in \mathbb{R}^{I} \mid \zeta \cdot \theta=0 \text { (resp. }>0,<0\right) \text { for } \theta \in R_{+}^{0}(\mathbf{v})\left(\text { resp. } R_{+}^{+}(\mathbf{v}), R_{+}^{-}(\mathbf{v})\right)\right\} .
$$

When we want to emphasize that it depends on the dimension vector $\mathbf{v}$, we call it a $\mathbf{v}$-face. A face is an open convex cone in the subspace $\left\{\zeta \in \mathbb{R}^{I} \mid \zeta \cdot \theta=0\right.$ for $\left.\theta \in R_{+}^{0}(\mathbf{v})\right\}$. A face is called a chamber (or $\mathbf{v}$-chamber) if it is an open subset in $\mathbb{R}^{I}$, i.e., $R_{+}^{0}(\mathbf{v})=\varnothing$. The closure of $F$ is

$$
\left.\bar{F}=\left\{\zeta \in \mathbb{R}^{I} \mid \zeta \cdot \theta=0 \text { (resp. } \geq 0, \leq 0\right) \text { for } \theta \in R_{+}^{0}(\mathbf{v})\left(\operatorname{resp} . R_{+}^{+}(\mathbf{v}), R_{+}^{-}(\mathbf{v})\right)\right\}
$$


Lemma 2.12. Suppose $W \neq 0$.

(1) (cf. [22, 2.8]) Suppose $\zeta$ is in a chamber. Then $\zeta$-semistability implies $\zeta$-stability, and hence $\mathfrak{M}_{\zeta}^{\mathrm{s}}(\mathbf{v}, \mathbf{w})=\mathfrak{M}_{\zeta}(\mathbf{v}, \mathbf{w})$.

(2) (cf. $[30,1.4])$ If two stability parameters $\zeta, \zeta^{\prime}$ are contained in the same face $F, \zeta$-stability (resp. $\zeta$-semistability) is equivalent to $\zeta^{\prime}$-stability (resp. $\zeta^{\prime}$-semistability).

(3) Suppose stability parameters $\zeta, \zeta^{\bullet}$ are contained in $F$ and $F^{\bullet}$ respectively. If $F^{\bullet} \subset \bar{F}$, then

(a) a $\zeta$-semistable module is $\zeta^{\bullet}$-semistable,

(b) $a \zeta^{\bullet}$-stable module is $\zeta$-stable.

Proof. (1) For given $V, W$, we define $\tilde{\zeta}=\left(\zeta, \zeta_{\infty}\right)$ with $\theta_{\tilde{\zeta}}(V, W)=0$ as before. Suppose that $(B, a, b)$ is $\tilde{\zeta}$-semistable, but not $\tilde{\zeta}$-stable. We take a Jordan-Hölder filtration as in Theorem 2.8(2). Since $\delta_{k} W / \delta_{k+1} W \neq 0$ only for one $k$ in $0, \ldots, N$, we have a $k$ with $\delta_{k} W / \delta_{k+1} W=0$ from the assumption $N \geq 1$. As the quotient module $\operatorname{gr}_{k}(B, a, b)=\left.(B, a, b)\right|_{\left(V^{k}, \delta_{k} W\right) /\left(V^{k+1}, \delta_{k+1} W\right)}$ is $\tilde{\zeta}$-stable, its dimension vector $\operatorname{dim} V^{k}-\operatorname{dim} V^{k+1}$ (with the $\infty$-component is 0 ) is in $R_{+}(\mathbf{v}$ ) by Lemma 2.11. Moreover, by the condition in the Jordan-Hölder filtration, we have

$$
\theta_{\tilde{\zeta}}\left(\operatorname{gr}_{k}(B, a, b)\right)=\theta_{\tilde{\zeta}}(V, W) .
$$

The right hand side is equal to 0 by our convention, and the left hand side is equal to $\zeta \cdot\left(\operatorname{dim} V^{k}-\right.$ $\left.\operatorname{dim} V^{k+1}\right)$ up to scalar. This contradicts with our assumption that $\zeta$ is in a chamber.

(2) We define $\tilde{\zeta}, \tilde{\zeta}^{\prime}$ as before. Suppose that $(B, a, b) \in \mu^{-1}(0) \subset \mathbf{M}(\mathbf{v}, \mathbf{w})$ is $\tilde{\zeta}$-semistable and is not $\tilde{\zeta}^{\prime}$-semistable. We take the Harder-Narasimhan filtration for $(B, a, b)$ with respect to the $\tilde{\zeta}^{\prime}$-stability as in Theorem 2.8(1). We have $N \geq 1$ from the assumption.

Consider first the case when there exists $0 \leq k \leq k_{W}-1$ with $\theta_{\tilde{\zeta}^{\prime}}\left(\operatorname{gr}_{k}(B, a, b)\right)<0$. Then $\theta_{\tilde{\zeta}^{\prime}}\left(\operatorname{gr}_{l}(B, a, b)\right)<0$ for any $0 \leq l \leq k$. On the other hand from the $\zeta$-semistability of $(B, a, b)$, we have $\theta_{\tilde{\zeta}}\left((V, W) /\left(V^{k}, \delta_{k} W\right)\right) \geq 0$ by Lemma 2.6. Therefore there exists at least one $l$ in $[0, k]$ with $\theta_{\tilde{\zeta}}\left(\operatorname{gr}_{l}(B, a, b)\right) \geq 0$. We further take a Jordan-Hölder filtration of $\operatorname{gr}_{l}(B, a, b)$ to find $\tilde{\zeta}^{\prime}$-stable representations $V^{\prime}$ with $\zeta^{\prime} \cdot \operatorname{dim} V^{\prime} / \sum \operatorname{dim} V_{i}^{\prime}=\theta_{\tilde{\zeta}^{\prime}}\left(V^{\prime}, 0\right)=\theta_{\tilde{\zeta}^{\prime}}\left(\operatorname{gr}_{l}(B, a, b)\right)<0$. Note that $\operatorname{gr}_{l}(B, a, b)$, and hence $V^{\prime}$ has 0 in the $W$-component. Since $\theta_{\tilde{\zeta}}\left(\operatorname{gr}_{l}(B, a, b)\right) \geq 0$, we can take $V^{\prime}$ so that $\zeta \cdot \operatorname{dim} V^{\prime} / \sum \operatorname{dim} V_{i}^{\prime}=\theta_{\tilde{\zeta}}\left(V^{\prime}, 0\right) \geq 0$. As $\operatorname{dim} V^{\prime} \in R_{+}(\mathbf{v})$, this contradicts with the assumption that $\zeta$ and $\zeta^{\prime}$ are in the common face.

The same argument leads to a contradiction in the case when there exists $k_{W}+1 \leq k \leq N$ with $\theta_{\tilde{\zeta}^{\prime}}\left(\operatorname{gr}_{k}(B, a, b)\right)>0$. Since $N \geq 1$ and $\theta_{\tilde{\zeta}^{\prime}}(V, W)=0$, at least one of two cases actually occur. Therefore $(B, a, b)$ is $\zeta^{\prime}$-semistable.

Suppose further that $(B, a, b)$ is $\zeta$-stable. We want to show that it is also $\zeta^{\prime}$-stable. Assume not, and take a Jordan-Hölder filtration of $(B, a, b)$ with respect to the $\zeta^{\prime}$-stability. Either of $\operatorname{gr}_{0}(B, a, b)$ and $\operatorname{gr}_{N}(B, a, b)$ has the $W$-component 0. Suppose the first one has the $W$ component 0. We have $\zeta^{\prime} \cdot \operatorname{dim}\left(V / V^{1}\right) / \sum \operatorname{dim} V_{i} / V_{i}^{1}=\theta_{\tilde{\zeta}^{\prime}}\left(\operatorname{gr}_{0}(B, a, b)\right)=0$. On the other hand, the $\zeta$-stability of $(B, a, b)$ implies $\zeta \cdot \operatorname{dim}\left(V / V^{1}\right) / \sum \operatorname{dim} V_{i} / V_{i}^{1}=\theta_{\tilde{\zeta}}\left(\operatorname{gr}_{0}(B, a, b)\right)>0$. This contradicts with the assumption. The same argument applies to the case when $\operatorname{gr}_{N}(B, a, b)$ has the $W$ component 0 . Therefore $(B, a, b)$ is $\zeta^{\prime}$-stable.

(3) Take a sequence $\left\{\zeta_{n}\right\}$ in $F$ converging to $\zeta \bullet$. Take a nonzero submodule $(S, \delta W)$ of $(B, a, b)$. (a) From the $\zeta$-semistability and (2), we have $\theta_{\tilde{\zeta}_{n}}(S, \delta W) \leq \theta_{\tilde{\zeta}_{n}}(V, W)$ for any $n$. Taking limit, we get $\theta_{\tilde{\zeta} \bullet}(S, \delta W) \leq \theta_{\tilde{\zeta} \bullet}(V, W)$. Therefore $(B, a, b)$ is $\zeta^{\bullet}$-semistable. (b) We assume $(S, \delta W) \neq(V, W)$. From the $\zeta^{\bullet}$-stability, we have $\theta_{\tilde{\zeta} \bullet}(S, \delta W)<\theta_{\tilde{\zeta} \bullet}(V, W)$. Then $\theta_{\tilde{\zeta}_{n}}(S, \delta W)<$ $\theta_{\tilde{\zeta}_{n}}(V, W)$ for sufficiently large $n$. Therefore $(B, a, b)$ is $\zeta_{n}$-stable. By $(2)$ it is also $\zeta$-stable.

Remark 2.13. When $W=0$, we need to modify the definitions of faces and chambers: Replace $\mathbb{R}^{I}$ by $\{\zeta \mid \zeta \cdot \mathbf{v}=0\}$, and $R_{+}(\mathbf{v})$ by $R_{+}(\mathbf{v}) \backslash\{\mathbf{v}\}$. Then the same proof works. 
From this lemma, we can define the $\zeta$-(semi)stability for $\zeta \in \mathbb{R}^{I}$, not necessarily in $\mathbb{Q}^{I}$, as it depends only on the face containing $\zeta$.

\section{$2.4 \quad$ Nonemptiness of $\mathfrak{M}_{\zeta}^{\mathrm{s}}$}

For most of purposes in our paper, Lemma 2.11 is enough, but we can use a rotation of the hyperKähler structure and then apply Crawley-Boevey's result [8] to get a necessary and sufficient condition for $\mathfrak{M}_{\zeta}^{\mathrm{s}}(\mathbf{v}, \mathbf{w}) \neq \varnothing$. This will be given in this subsection.

Suppose that the graph $(I, E)$ does not have edge loops. We thus associate a Kac-Moody Lie algebra $\mathfrak{g}$ to $(I, E)$. We fix a dimension vector $\mathbf{w}=\left(w_{i}\right) \in \mathbb{Z}_{\geq 0}^{I}$, which is considered as a dominant weight for $\mathfrak{g}$. Let $(\tilde{I}, \tilde{E})$ be a graph obtained from $(I, E)$ by adding a new vertex $\infty$ and $w_{i}$ edges between $\infty$ and $i$. The new graph $(\tilde{I}, \tilde{E})$ defines another Kac-Moody Lie algebra which we denote by $\tilde{\mathfrak{g}}$. We denote the corresponding Cartan matrix by $\tilde{\mathbf{C}}$. This new quiver was implicitly used in Section 2.2.

Lemma 2.14. Let $\alpha_{\infty}$ denote the simple root corresponding to the vertex $\infty$. Let $V$ be the direct sum of root spaces $\tilde{\mathfrak{g}}_{\alpha}$ of $\tilde{\mathfrak{g}}$, where $\alpha$ is of the form $\alpha=-\sum_{i \in I} m_{i} \alpha_{i}-\alpha_{\infty}$. Let $\mathfrak{g}$ act on $V$ by the restriction of the adjoint representation. Then $V$ is isomorphic to the irreducible integrable highest weight representation $V(\mathbf{w})$ with the highest weight vector $f_{\infty}$ of weight $\mathbf{w}$. In particular, the root multiplicity $\operatorname{dim} \tilde{\mathfrak{g}}_{\alpha}$ is equal to the weight multiplicity of $\left.\alpha\right|_{\mathfrak{h}}$ in $V(\mathbf{w})$, where $\mathfrak{h}$ is the Cartan subalgebra of $\mathfrak{g}$.

Proof. From the definition we have

$$
\left[e_{i}, f_{\infty}\right]=0, \quad\left[h_{i}, f_{\infty}\right]=w_{i} f_{\infty} \quad \text { for } i \in I
$$

Moreover $\tilde{\mathfrak{g}}_{\alpha}$ is a linear span of the elements of the form

$$
\left[f_{i_{1}},\left[f_{i_{2}},\left[\ldots,\left[f_{i_{k-1}},\left[f_{\infty},[\underbrace{\left.f_{i_{k}},\left[\ldots\left[f_{i_{s-1}}, f_{i_{s}}\right] \ldots\right]\right]}_{=x}]\right] \ldots\right]\right]\right]=-\left[f_{i_{1}},\left[f_{i_{2}},\left[\ldots,\left[f_{i_{k-1}},\left[x, f_{\infty}\right]\right] \ldots\right]\right]\right]\right.
$$

for $i_{1}, i_{2}, \ldots \in I$. This means that $V$ is a highest weight module. We also know $V$ is integrable by $[12,3.5]$. Now the assertion follows from $[12,10.4]$.

Theorem 2.15. (1) Suppose $W=0$ and consider a dimension vector $\mathbf{v}$ with $\zeta \cdot \mathbf{v}=0$. Then $\mathfrak{M}_{\zeta}^{\mathrm{s}}(\mathbf{v}, 0) \neq \varnothing$ if and only if the following holds:

- $\mathbf{v}$ is a positive root, and $p(\mathbf{v})>\sum_{t=1}^{r} p\left(\beta^{(t)}\right)$ for any decomposition $\mathbf{v}=\sum_{t=1}^{r} \beta^{(t)}$ with $r \geq 2$ and $\beta^{(t)}$ a positive root with $\zeta \cdot \beta^{(t)}=0$ for all $t$,

where $p(x)=1-\frac{1}{2}^{t} x \mathbf{C} x$.

(2) Suppose $\mathbf{w} \neq 0$. Then $\mathfrak{M}_{\zeta}^{\mathrm{s}}(\mathbf{v}, \mathbf{w}) \neq \varnothing$ if and only if the following holds:

- $\mathbf{w}-\mathbf{v}$ is a weight of the integrable highest weight representation $V(\mathbf{w})$ of the highest weight $\mathbf{w}$, and ${ }^{t} \mathbf{v}\left(\mathbf{w}-\frac{1}{2} \mathbf{C v}\right)>{ }^{t} \mathbf{v}^{0}\left(\mathbf{w}-\frac{1}{2} \mathbf{C v}^{0}\right)+\sum_{t=1}^{r} p\left(\beta^{(t)}\right)$ for any decomposition $\mathbf{v}=$ $\mathbf{v}^{0}+\sum_{t=1}^{r} \beta^{(t)}$ with $r \geq 1, \mathbf{w}-\mathbf{v}^{0}$ is a weight of $V(\mathbf{w})$, and $\beta^{(t)}$ a positive root with $\zeta \cdot \beta^{(t)}=0$ for all $t$.

Remark 2.16. For more general pairs of parameters $\left(\zeta, \zeta_{\mathbb{C}}\right)$, it seems natural to expect that the existence of $\zeta$-stable module $B$ with $\mu(B, a, b)=\zeta_{\mathbb{C}}$ is equivalent to the above condition with $\zeta \cdot \beta^{(t)}=0$ replaced by ' $\zeta \cdot \beta^{(t)}=0$ and $\zeta_{\mathbb{C}} \cdot \beta^{(t)}=0$ '. 
Proof. (1) A $\zeta$-stable module with $\mu=0$ corresponds to a point satisfying the hyper-Kähler moment map $\left(\mu_{\mathbb{R}}, \mu\right)=(\zeta, 0)$, which correspond a 0 -stable module (i.e., a simple module) with $\mu=\zeta$ after a rotation of the complex structures. Then by [8, Theorem 1.2] such a simple module exists if and only if the above condition holds.

(2) For the given $\mathbf{w}$ we construct the quiver $(\tilde{I}, \tilde{E})$ as above. We consider the dimension vector $\tilde{\mathbf{v}}=\mathbf{v}+\alpha_{\infty}$ and define the stability parameter $\tilde{\zeta}=\left(\zeta, \zeta_{\infty}\right)$ by $\zeta \cdot \operatorname{dim} \mathbf{v}+\zeta_{\infty}=0$. Then $\mathfrak{M}_{\zeta}^{\mathrm{s}}(\mathbf{v}, \mathbf{w})$ is isomorphic to the quiver variety $\mathfrak{M}_{\tilde{\zeta}}^{\text {s }}(\tilde{\mathbf{v}}, 0)$ associated with $(\tilde{I}, \tilde{E})$ [8, the end of Introduction]. Therefore we can apply the criterion in (1) together with the observation Lemma 2.14. We first note that

$$
p(\tilde{\mathbf{v}})=1-\frac{1}{2}{ }^{t} \tilde{\mathbf{V}} \tilde{\mathbf{V}} \tilde{\mathbf{v}}={ }^{t} \mathbf{v}\left(\mathbf{w}-\frac{1}{2} \mathbf{C v}\right) .
$$

Also if we have a decomposition $\tilde{\mathbf{v}}=\sum \beta^{(t)}$, one of $\beta^{(t)}$ has 1 in the entry $\infty$, and other $\beta^{(t)}$ 's have 0 in the entry $\infty$. We rewrite the former as $\mathbf{v}^{0}+\alpha_{\infty}$, and identify the latter with positive roots for $(I, E)$. Now the assertion follows from (1).

\subsection{Partial resolutions}

Let $\zeta, \zeta^{\bullet} \in \mathbb{R}^{I}$ as in Lemma 2.12(3). Then we have a morphism

$$
\pi_{\zeta}^{\bullet}, \zeta: \mathfrak{M}_{\zeta} \rightarrow \mathfrak{M}_{\zeta}
$$

thanks to Lemma 2.12(3)(a). By Lemma 2.12(3)(b) it is an isomorphism on the preimage $\pi_{\zeta^{\bullet}, \zeta}^{-1}\left(\mathfrak{M}_{\zeta^{\bullet}}^{\mathrm{s}}\right)$.

If further $\zeta^{\circ}, \zeta^{\bullet}$ are as in Lemma $2.12(3), \zeta, \zeta^{\bullet}$ are also, and we have $\pi_{\zeta \bullet, \zeta}=\pi_{\zeta^{\bullet}, \zeta^{\circ}} \circ \pi_{\zeta^{\circ}, \zeta}$.

Since 0 is always in the closure of any face, we always have a morphism $\pi_{0, \zeta}: \mathfrak{M}_{\zeta} \rightarrow \mathfrak{M}_{0}$ for any $\zeta^{\bullet}$. There always exists a chamber $\mathcal{C}$ containing $\zeta^{\bullet}$ in the closure. If we take a parameter $\zeta$ from $\mathcal{C}$, we have $\pi_{\zeta} \bullet, \mathfrak{M}_{\zeta} \rightarrow \mathfrak{M}_{\zeta} \bullet$. And we have $\pi_{0, \zeta}=\pi_{0, \zeta} \bullet \pi_{\zeta, \zeta}$. Since $\zeta$ is in a chamber, we have $\mathfrak{M}_{\zeta}=\mathfrak{M}_{\zeta}^{\mathrm{s}}$, and hence $\mathfrak{M}_{\zeta}$ is nonsingular. It is known that $\mathfrak{M}_{\zeta}$ is a resolution of singularities of $\mathfrak{M}_{0}$ in many cases (e.g., if $\mathfrak{M}_{\zeta}^{\mathrm{s}} \neq \varnothing$ (see [22, Theorem 4.1])). In these cases, $\mathfrak{M}_{\zeta} \bullet$ is a partial resolution of singularities of $\mathfrak{M}_{0}$.

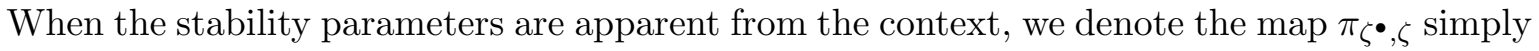
by $\pi$.

\subsection{Stratum}

We recall the stratification on $\mathfrak{M}_{\zeta}$ considered in [22, Section 6], [24, Section 3].

Suppose that $(B, a, b)$ is $\zeta$-polystable. Let us decompose it as

$$
\begin{aligned}
& V \cong V^{0} \oplus\left(V^{1}\right)^{\oplus \widehat{v}_{1}} \oplus \cdots \oplus\left(V^{r}\right)^{\oplus \widehat{v}_{r}}, \\
& (B, a, b) \cong\left(B^{0}, a^{0}, b^{0}\right) \oplus\left(B^{1}\right)^{\oplus \widehat{v}_{1}} \oplus \cdots \oplus\left(B^{r}\right)^{\oplus \widehat{v}_{r}},
\end{aligned}
$$

where $\left(B^{0}, a^{0}, b^{0}\right) \in \mu^{-1}(0) \cap \mathbf{M}\left(V^{0}, W\right)$ is the unique factor having $W \neq 0$, and $B^{k} \in \mu^{-1}(0) \cap$ $\mathrm{E}\left(V^{k}, V^{k}\right)(k=1, \ldots, r)$ are pairwise non-isomorphic $\zeta$-stable modules and $\widehat{v}_{k}$ is its multiplicity in $(B, a, b)$. (See $[22,6.5],[24,3.27]$.)

The stabilizer $\widehat{G}$ of $(B, a, b)$ is conjugate to

$$
\left\{\operatorname{id}_{V^{0}}\right\} \times \prod_{k=1}^{r}\left(\mathrm{GL}\left(\widehat{v}_{k}\right) \otimes \mathrm{id}_{V^{k}}\right) .
$$

Conversely if the stabilizer is conjugate to this subgroup, the module has the decomposition above. 
We thus have a stratification by $G$-orbit type:

$$
\mathfrak{M}_{\zeta}=\bigsqcup_{(\widehat{G})}\left(\mathfrak{M}_{\zeta}\right)_{(\widehat{G})},
$$

where $\left(\mathfrak{M}_{\zeta}\right)_{(\widehat{G})}$ consists of modules whose stabilizers are conjugate to a subgroup $\widehat{G}$ of $G$.

A list of strata can be given by Theorem 2.15 in principle, but to use this result, we need to know all roots, and it is not so easy in general. The following definition was considered in [26, 2.6.4] to avoid this difficulty and concentrate only on the stratum with $\mathbf{w} \neq 0$.

Definition 2.18. A stratum $\left(\mathfrak{M}_{\zeta}\right)_{(\widehat{G})}$ is regular if it is of the form $\mathfrak{M}_{\zeta}^{\mathrm{s}}\left(\mathbf{v}^{\prime}, \mathbf{w}\right) \times\left\{\bigoplus_{i \in I} S_{i}^{\oplus\left(v_{i}-v_{i}^{\prime}\right)}\right\}$ for a $\mathbf{v}^{\prime}=\left(v_{i}^{\prime}\right)$, where $S_{i}$ is the module with $\mathbb{C}$ on the vertex $i$, and 0 on the other vertices and $B=0$. Here we assume $\zeta_{i}=0$.

A point $x \in \mathfrak{M}_{\zeta}(\mathbf{v}, \mathbf{w})$ is regular if it is contained in a regular stratum.

If the graph is of finite type, all strata in $\mathfrak{M}_{0}(\mathbf{v}, \mathbf{w})$ are regular. This was proved in [22, 6.7], but it also follows from Theorem 2.15, as $p(x)=0$ for a real root $x$.

\section{$2.7 \quad$ Local structure}

Let $\zeta, \zeta^{\bullet}$ be as in Lemma 2.12(3). We examine the local structure of $\mathfrak{M}_{\zeta} \bullet$ (resp. $\mathfrak{M}_{\zeta}$ ) around a point $x$ (resp. $\left.\pi_{\zeta \bullet, \zeta}^{-1}(x)\right)$ in this subsection. This has been done in [22, Section 6], [26, Section 3.2], but we explain the results in a slightly different form.

Let us take a representative $(B, a, b)$ of $x$, which is $\zeta^{\bullet}$-polystable. We consider the complex $($ see $[24,(3.11)])$

$$
\begin{aligned}
& \mathscr{C} \bullet: \mathrm{L}(V, V) \stackrel{\alpha}{\rightarrow} \mathrm{E}(V, V) \oplus \mathrm{L}(W, V) \oplus \mathrm{L}(V, W) \stackrel{\beta}{\rightarrow} \mathrm{L}(V, V), \\
& \alpha(\xi)=(B \xi-\xi B) \oplus(-\xi a) \oplus(b \xi), \quad \beta(C, d, e)=\varepsilon B C+\varepsilon C B+a e+d b,
\end{aligned}
$$

where $\alpha$ is the infinitesimal action of the Lie algebra of $G_{V}$ on $\mathbf{M}$, and $\beta$ is the differential of the moment map $\mu$ at $(B, a, b)$.

Suppose that $(B, a, b)$ is decomposed into a direct sum of $\zeta^{\bullet}$-stable modules as in (2.17). Then $\operatorname{Ker} \beta / \operatorname{Im} \alpha$ is the symplectic normal denoted by $\widehat{\mathbf{M}}$ in [22, Section 6], [26, Section 3.2]. The complex $\mathscr{C} \bullet$ decomposes as $\mathscr{C} \bullet=\bigoplus_{k, l=0}^{r}\left(\mathscr{C}_{k, l}^{\bullet}\right)^{\oplus \widehat{v}_{k} \widehat{v}_{l}}$ with

$$
\mathscr{C}_{k, l}^{\bullet}: \mathrm{L}\left(V^{k}, V^{l}\right) \stackrel{\alpha}{\rightarrow} \mathrm{E}\left(V^{k}, V^{l}\right) \oplus \mathrm{L}\left(W, V^{l}\right)^{\oplus \delta_{k 0}} \oplus \mathrm{L}\left(V^{k}, W\right)^{\oplus \delta_{0 l}} \stackrel{\beta}{\rightarrow} \mathrm{L}\left(V^{k}, V^{l}\right)
$$

where $\mathrm{L}\left(W, V^{l}\right)$ appears in case $k=0$ and $\mathrm{L}\left(V^{k}, W\right)$ in case $l=0$. We also put $\widehat{v}_{0}=1$. Then it is easy to show that $\operatorname{Ker} \alpha=0$ unless $k=l \neq 0$ and Ker $\alpha=\mathbb{C}$ id for $k=l \neq 0$, and the similar statement for Coker $\beta$ : Note that $\operatorname{Ker} \alpha$ is the space of homomorphisms between $\zeta^{\bullet}$-stable modules, and Coker $\beta$ is its dual. Then a standard argument, comparing $\theta_{\tilde{\zeta} \bullet \text {-values of }}$ Ker $\xi$ and $\operatorname{Im} \xi$ of a homomorphism $\xi$ and using Lemma 2.6, implies the assertion. We remark that a complex used often in [24] (see (2.27)) is an example of $\mathscr{C}_{k l}^{\bullet}$ where $V^{k}$ is $S_{i}$, a module with $\mathbb{C}$ on the vertex $i \in I$, and 0 on the other vertices and $B=0$.

We construct a new graph with $\widehat{I}=\{1, \ldots, r\}$ with the associated Cartan matrix $\widehat{\mathbf{C}}=\left(\widehat{c}_{k l}\right)$ by $\widehat{c}_{k l} \stackrel{\text { def }}{=} 2 \delta_{k l}-\operatorname{dim} \operatorname{Ker} \beta / \operatorname{Im} \alpha$ for the complex $\mathscr{C}_{k l}^{\bullet}$. This is equal to the alternating sum of the dimensions of terms, i.e., $={ }^{t} \mathbf{v}^{k} \mathbf{C v}^{l}$ by the above discussion. Note $\widehat{a}_{k l}=\widehat{a}_{l k}$. We also put

$$
\hat{V}_{k} \stackrel{\text { def }}{=} \mathbb{C}^{\widehat{v}_{k}}, \quad \hat{W}_{k} \stackrel{\text { def }}{=} \operatorname{Ker} \beta / \operatorname{Im} \alpha \quad \text { for } \mathscr{C}_{k 0}^{\bullet},
$$

and consider $\mathbf{M}(\hat{V}, \hat{W})$ defined for the new graph with the $\widehat{I}$-graded vector spaces $\hat{V}$, $\hat{W}$. The stabilizer $\widehat{G}$ of $(B, a, b)$ is naturally isomorphic to $\prod_{k \in \hat{I}} \mathrm{GL}\left(\hat{V}_{k}\right)$. It acts on $\mathbf{M}(\hat{V}, \hat{W})$. 
We have the moment map $\widehat{\mu}: \mathbf{M}(\hat{V}, \hat{W}) \rightarrow \mathrm{L}(\hat{V}, \hat{V}) \cong \operatorname{Lie}(\widehat{G})^{*}$. We consider the quotient $\widehat{\mathfrak{M}}_{0}(\hat{V}, \hat{W})=\widehat{\mu}^{-1}(0) / / \widehat{G}$, where the stability parameter is 0 . We also consider $\widehat{\mathfrak{M}}_{\zeta}(\hat{V}, \hat{W})$, where the stability parameter $\zeta$ is considered as a $\mathbb{R}$-character $\chi_{\zeta}$ of $\widehat{G}$ through the inclusion $\widehat{G} \subset G$. There is a morphism $\widehat{\pi}: \widehat{\mathfrak{M}}_{\zeta}(\hat{V}, \hat{W}) \rightarrow \widehat{\mathfrak{M}}_{0}(\hat{V}, \hat{W})$. Then $[26,3.2 .1] \mathfrak{M}_{\zeta}(V, W)$, around $x=[B, a, b]$ a point corresponding to $(B, a, b)$, is locally isomorphic to a neighbourhood of 0 in $\widehat{\mathfrak{M}}_{0}^{\text {norm }}(\hat{V}, \hat{W}) \times T$, where $\widehat{\mathfrak{M}}_{0}^{\text {norm }}(\hat{V}, \hat{W})$ is as in $(2.3), T$ is the product of the trivial factor $\widehat{\mathbf{M}}^{\mathrm{el}}(\hat{V}, \hat{W})$ (see $(2.1)$ ) and $\hat{W}_{0}=\operatorname{Ker} \beta / \operatorname{Im} \alpha$ for the complex $\mathscr{C}_{00}^{\bullet}$ which is isomorphic to the tangent space $T_{\left[\left(B^{0}, a^{0}, b^{0}\right)\right]} \mathfrak{M}_{\zeta^{\bullet}}\left(V^{0}, W\right)$. We also have a lifting of the local isomorphism to $\mathfrak{M}_{\zeta}(V, W)$ and $\widehat{\mathfrak{M}}_{\zeta}(\hat{V}, \hat{W})$, and hence the following commutative diagram:

$$
\begin{aligned}
& \mathfrak{M}_{\zeta}(V, W) \supset \pi^{-1}(U) \stackrel{\tilde{\Phi}}{\cong} \widehat{\pi}^{-1}(\hat{U}) \times U_{0} \quad \subset \widehat{\mathfrak{M}}_{\zeta}^{\text {norm }}(\hat{V}, \hat{W}) \times T \\
& \pi \downarrow \quad \downarrow \widehat{\pi} \times \text { id }
\end{aligned}
$$

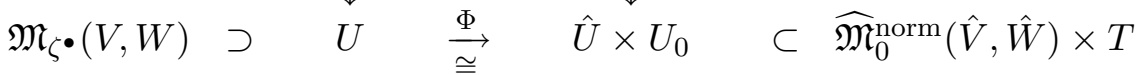

$$
\begin{aligned}
& \Psi \quad \psi \\
& x \quad \mapsto \quad 0
\end{aligned}
$$

Note that the factor $T$ is the tangent space to the stratum $\left(\mathfrak{M}_{\zeta} \bullet(V, W)\right)_{(\widehat{G})}$ containing $x$. (Remark: $[26,3.2 .1]$ states this result for $\zeta^{\bullet}=0$, but the same proof works. To see that the stability parameter for $\widehat{\mathfrak{M}}_{\zeta} \bullet$ becomes 0 , we note also that the restriction of the character $\chi_{\zeta} \bullet$ to $\widehat{G}$ is trivial.)

The following formula will be useful:

$$
\widehat{w}_{k}-\sum_{l \neq 0} \widehat{c}_{k l} \widehat{v}_{l}={ }^{t} \mathbf{v}^{k}(\mathbf{w}-\mathbf{C v})
$$

Remark 2.22. The Cartan matrix $\widehat{\mathbf{C}}=\left(\widehat{c}_{k l}\right)$ is given by the formula $\widehat{c}_{k l}={ }^{t} \mathbf{v}^{k} \mathbf{C} \mathbf{v}^{l}$, where $\mathbf{v}^{k}=\operatorname{dim} V^{k}$. Suppose that the original graph is of affine type. Then $\mathbf{C}$ is positive semidefinite and the kernel is spanned by $\delta$. Therefore $\mathbf{v}^{k}$ with ${ }^{t} \mathbf{v}^{k} \mathbf{C v}^{k}=0$ is a multiple of $\delta$, hence $\widehat{c}_{k l}=0$ for any $l \in \widehat{I}$. This means that a connected component of the graph $(\widehat{I}, \widehat{\mathbf{C}})$ is either a graph of affine or finite type (without edge loops), or a graph with a single vertex and a single edge loop (i.e., the Jordan quiver). If the original graph is of finite type, then we only get a graph of finite type as $\mathbf{C}$ is positive definite.

As an application, we obtain the following:

Proposition 2.23 (cf. [22, 6.11], [24, 10.4, 10.11]). Let $\zeta, \zeta$ be as above and consider $\pi: \mathfrak{M}_{\zeta}(V, W) \rightarrow \mathfrak{M}_{\zeta}(V, W)$. We further assume that $\zeta$ is in a chamber so that $\mathfrak{M}_{\zeta}(V, W)$ is nonsingular.

(1) Take a point $x$ in a stratum $\left(\mathfrak{M}_{\zeta^{\bullet}}\right)_{(\widehat{G})}$. If $\pi^{-1}(x) \neq \varnothing$, then we have

$$
\operatorname{dim} \pi^{-1}(x) \leq \frac{1}{2}\left(\operatorname{dim} \mathfrak{M}_{\zeta}(V, W)-\operatorname{dim}\left(\mathfrak{M}_{\zeta}\right)_{(\widehat{G})}\right) .
$$

(2) Replace the target of $\pi$ by the image, hence consider $\pi: \mathfrak{M}_{\zeta}(V, W) \rightarrow \pi\left(\mathfrak{M}_{\zeta}(V, W)\right)$. It is semismall with respect to the stratification $\pi\left(\mathfrak{M}_{\zeta}(V, W)\right)=\bigsqcup\left(\mathfrak{M}_{\zeta}\right)_{(\widehat{G})}$ where $\widehat{G}$ runs over the conjugacy classes of subgroups of $G_{V}$ such that $\left(\mathfrak{M}_{\zeta}\right)_{(\widehat{G})}$ is contained in $\pi\left(\mathfrak{M}_{\zeta}(V, W)\right)$.

Proof. (1) This was proved under the assumption $\mathfrak{M}_{\zeta}^{\mathrm{s}}(V, W) \neq \varnothing$ in $[22,6.11]$, but its proof actually gives the above. The point of the proof was that the fiber $\widehat{\pi}^{-1}(0)$ is a subvariety in $\widehat{\mathfrak{M}}_{\zeta}(\hat{V}, \hat{W})$ (divided by the trivial factor $(2.1)$ ), which is an affine algebraic manifold by Theorem 2.10. 
(2) By (2.20) and the subsequent remark, for each stratum $\left(\mathfrak{M}_{\zeta^{\bullet}}\right)_{(\widehat{G})}$, the restriction of $\pi$ to the preimage $\pi^{-1}\left(\left(\mathfrak{M}_{\zeta \bullet}\right)_{(\widehat{G})}\right)$ is a fiber bundle. Moreover, by $(1)$, there exists a stratum $\left(\mathfrak{M}_{\zeta}\right)_{(\widehat{G})}$ such that $\operatorname{dim}\left(\mathfrak{M}_{\zeta}\right)_{(\widehat{G})}=\operatorname{dim} \mathfrak{M}_{\zeta}(V, W)$. Therefore we have $\operatorname{dim} \pi\left(\mathfrak{M}_{\zeta}(V, W)\right)=\operatorname{dim} \mathfrak{M}_{\zeta}(V, W)$. Hence (1) implies the assertion.

Remark 2.24. In $[24,10.11]$ we claimed a stronger statement that all strata are relevant in the statement (2) of Proposition 2.23 when the graph is of finite type. This follows from the above observation (1) and the fact that $\widehat{\pi}^{-1}(0)$ is exactly half-dimensional in $\widehat{\mathfrak{M}}_{\zeta}(\hat{V}, \hat{W})$ if the graph does not contains edge loops [22, 5.8].

Now consider the case when the graph is of affine type. By the above observation (1), it is enough to show that the fiber $\widehat{\pi}^{-1}(0)$ in $\widehat{\mathfrak{M}}_{\zeta}^{\text {norm }}(\hat{V}, \hat{W}) \rightarrow \widehat{\mathfrak{M}}_{0}^{\text {norm }}(\hat{V}, \hat{W})$ is exactly halfdimensional. It is clearly enough to prove this assertion for each connected component of the graph $(\widehat{I}, \widehat{\mathbf{C}})$. Since it is known when the component has no edge loops, it is enough to consider the case when the component is the Jordan quiver. Since the trivial factor $\widehat{\mathbf{M}}^{\mathrm{el}}(\hat{V}, \hat{W})$ is 2dimensional (except for the trivial case $\hat{V}=0$ ), this means $\operatorname{dim} \widehat{\pi}^{-1}(0)=\operatorname{dim} \widehat{\mathfrak{M}}_{\zeta}(\hat{V}, \hat{W}) / 2-1$. This is known. (See [25, Exercise 5.15] and the references therein.) Therefore all strata are relevant also in the affine case.

For general quivers, the author does not know whether the same result holds or not.

\subsection{Example: Levi factors of parabolic subalgebras}

We give an example of a face, which will be related to the restriction to the Levi factor of a parabolic subalgebra.

Consider the chamber $\mathcal{C}=\left\{\zeta \in \mathbb{R}^{I} \mid \zeta_{i}>0\right.$ for all $\left.i \in I\right\}$. This corresponds to the stability condition $\zeta$ in Example 2.7(1). A face $F$ contained in the closure $\overline{\mathcal{C}}$ is of a form

$$
F=\left\{\zeta^{\bullet} \in \mathbb{R}^{I} \mid \zeta_{i}^{\bullet}=0(\text { resp. }>0) \text { for } i \in I^{0}\left(\text { resp. } I^{+}\right)\right\}
$$

for a disjoint decomposition $I=I^{0} \sqcup I^{+}$. We allow the cases $I^{0}=\varnothing$, i.e., $F=\mathcal{C}, \zeta^{\bullet}=\zeta$ and $I^{0}=I$, i.e., $\zeta^{\bullet}=0$. We have

$$
\mathfrak{M}_{\zeta}(\mathbf{v}, \mathbf{w}) \stackrel{\pi_{\zeta}, \zeta}{\longrightarrow} \mathfrak{M}_{\zeta} \bullet(\mathbf{v}, \mathbf{w}) \stackrel{\pi_{0, \zeta}}{\longrightarrow} \mathfrak{M}_{0}(\mathbf{v}, \mathbf{w})
$$

Let us describe strata of $\mathfrak{M}_{\zeta} \bullet(\mathbf{v}, \mathbf{w})$ in this example.

Proposition 2.25. (1) The strata are of the form

$$
\mathfrak{M}_{\zeta}^{\mathrm{s}}\left(\mathbf{v}^{0}, \mathbf{w}\right) \times\left(\mathfrak{M}_{0}\right)_{(\widehat{G})},
$$

where $\mathbf{v}-\mathbf{v}^{0}$ is supported on $I^{0}$, and $\left(\mathfrak{M}_{0}\right)_{(\widehat{G})}$ is a stratum of $\mathfrak{M}_{0}\left(\mathbf{v}-\mathbf{v}^{0}, 0\right)$ for the subgraph $I^{0}$, extended to the whole graph by 0 .

(2) If $\mathfrak{M}_{\zeta}^{\mathrm{s}}\left(\mathbf{v}^{0}, \mathbf{w}\right) \neq \varnothing$, we have $\left\langle h_{i}, \mathbf{w}-\mathbf{v}^{0}\right\rangle \geq 0$ for $i \in I^{0}$.

We say a weight $\lambda$ is $I^{0}$-dominant if $\left\langle h_{i}, \lambda\right\rangle \geq 0$ for all $i \in I^{0}$.

Proof. (1) A closed point in $\mathfrak{M}_{\zeta}(\mathbf{v}, \mathbf{w})$ is represented by a $\zeta^{\bullet}$-polystable module. Let $B \in$ $\mathfrak{M}_{\zeta^{\bullet}}^{\bullet}\left(\mathbf{v}^{\prime}, 0\right)$ be its component with $W=0$. As we have the constraint $\zeta^{\bullet} \cdot \mathbf{v}^{\prime}=0$, our choice of $\zeta^{\bullet}$ implies that $\mathbf{v}^{\prime}$ is supported on $I^{0}$. Thus $\zeta^{\bullet}$-stable modules are 0 -stable (i.e., simple) modules for the subgraph $I^{0}$, extended by 0 . 
(2) is implicitly proved in Section 2.7. Let us make it more apparent. Consider the complex $\mathscr{C}_{k l}^{\bullet}$ for a point $[B, a, b] \in \mathfrak{M}_{\zeta}^{\mathrm{s}}\left(\mathbf{v}^{0}, \mathbf{w}\right)$ and $S_{i}$, a module with $\mathbb{C}$ on the vertex $i \in I^{0}$, and 0 on the other vertices and $B=0$. Explicitly it is given by (see [24, (4.2)]):

$$
\begin{aligned}
& V_{i} \stackrel{\alpha_{i}}{\longrightarrow} \bigoplus_{h: \mathrm{i}(h)=i} V_{\mathrm{o}(h)} \oplus W_{i} \stackrel{\beta_{i}}{\longrightarrow} V_{i}, \\
& \alpha_{i} \stackrel{\text { def }}{=} \bigoplus_{\mathrm{i}(h)=i} B_{\bar{h}} \oplus b_{i}, \quad \beta_{i} \stackrel{\text { def }}{=}\left[\sum_{\mathrm{i}(h)=i} \varepsilon(h) B_{h} \quad a_{i}\right] .
\end{aligned}
$$

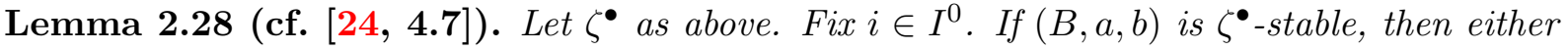
of the following hold:

(a) $W=0$ (hence $a=b=0$ ), and $B$ is the simple module $S_{i}$.

(b) The map $\alpha_{i}$ is injective and $\beta_{i}$ is surjective.

Since $W=0$ is excluded in (b), this gives the proof of the proposition.

Proof. This is a standard argument. The kernel of $\alpha_{i}$ gives a homomorphism from $S_{i}$ to $(B, a, b)$. Since both are $\zeta^{\bullet}$-stable and have the same $\theta_{\widehat{\zeta} \bullet}$ (both are 0 ), the homomorphism is either 0 or an isomorphism. Similarly the cokernel of $\beta_{i}$ is the dual of the space of homomorphisms from $(B, a, b)$ to $S_{i}$. Thus we have the same assertion.

Let us consider the restriction of $\pi_{0, \zeta}$ to the closure of the stratum $\mathfrak{M}_{\zeta}^{\mathrm{s}}\left(\mathbf{v}^{0}, \mathbf{w}\right) \times\left(\mathfrak{M}_{0}\right)_{(\widehat{G})}$ (in $\mathfrak{M}_{\zeta} \bullet(\mathbf{v}, \mathbf{w})$ ). It fits in the commutative diagram

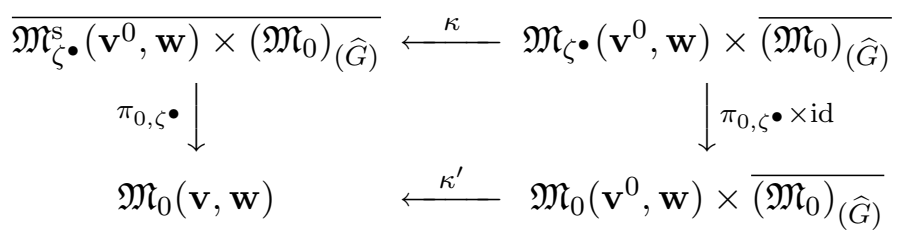

where the horizontal arrows are given by $\left([B, a, b],\left[B^{\prime}\right]\right) \mapsto\left[(B, a, b) \oplus B^{\prime}\right]$. They are finite morphisms. The upper horizontal arrow $\kappa$ is an isomorphism on the preimage of $\mathfrak{M}_{\zeta}^{\mathrm{s}}\left(\mathbf{v}^{0}, \mathbf{w}\right) \times$ $\left(\mathfrak{M}_{0}\right)_{(\widehat{G})}$. This means that a study of a stratum can be reduced to the special case when it is of the form $\mathfrak{M}_{\zeta^{\bullet}}^{\mathrm{s}}\left(\mathbf{v}^{0}, \mathbf{w}\right)$.

For a general graph, we can use Theorem 2.15 in principle, but to apply this result, we need to know all roots, and it is not so easy in general. Therefore we restrict ourselves to the case when the graph is of affine type from now until the end of this section.

Proposition 2.30 (cf. $[24, \mathbf{1 0 . 5}, \mathbf{1 0 . 8}]$ ). Suppose the graph is of affine type and $\mathbf{w} \neq 0$.

(1) Suppose $I^{0}=I$ and ${ }^{t} \delta \mathbf{w}=1$. Then $\mathfrak{M}_{\zeta}^{\mathbf{s}}(\mathbf{v}, \mathbf{w})=\varnothing$ unless $\mathbf{v}=0$.

(2) Suppose otherwise. Then $\mathfrak{M}_{\zeta}^{\mathrm{s}}(\mathbf{v}, \mathbf{w}) \neq \varnothing$ if and only if $\mathbf{w}-\mathbf{v}$ is an $I^{0}$-dominant weight of the irreducible integrable highest weight module $V(\mathbf{w})$.

Proof. (1) We have $\zeta^{\bullet}=0$ in this case.

Let us first give a proof based on the interpretation of $\mathfrak{M}_{0}^{\mathrm{s}}(\mathbf{v}, \mathbf{w})$ as moduli of vector bundles. Let $\Gamma$ be the finite subgroup of $\mathrm{SL}_{2}(\mathbb{C})$ corresponding to the graph via the McKay correspondence. Then $\mathfrak{M}_{0}^{\mathrm{s}}(\mathbf{v}, \mathbf{w})$ parametrizes framed $\Gamma$-equivariant vector bundles over $\mathbb{P}^{2}[25]$. Since $\mathbf{w}$ is 1-dimensional, it parametrizes line bundles. As the existence of the framing implies $c_{1}=0$, the line bundles are trivial. In particular, we have $\mathbf{v}=0$. 
Let us give another proof by using Theorem 2.15. The weights $\mathbf{w}-\mathbf{v}$ of the level 1 representation $V(\mathbf{w})$ are of the form $\mathbf{w}-\mathbf{v}^{0}-n \delta$, where $\mathbf{w}-\mathbf{v}^{0} \in W \cdot \mathbf{w}, n \in \mathbb{Z}_{\geq 0}$. (Here $W$ is the affine Weyl group.) Then

$$
{ }^{t} \mathbf{v}\left(\mathbf{w}-\frac{1}{2} \mathbf{C v}\right)={ }^{t} \mathbf{v}^{0}\left(\mathbf{w}-\frac{1}{2} \mathbf{C v}^{0}\right)+n={ }^{t} \mathbf{v}^{0}\left(\mathbf{w}-\frac{1}{2} \mathbf{C v}^{0}\right)+n p(\delta) .
$$

This violates the inequality in Theorem 2.15 unless $n=0$. But we also know that $\mathbf{w}-\mathbf{v}^{0}$ is dominant by Lemma 2.28. Hence $\mathbf{w}-\mathbf{v}^{0}=\mathbf{w}$, i.e., $\mathbf{v}^{0}=0$.

(2) This is proved in $[24,10.5,10.8]$ in the special case $I^{0}=I$. The argument works in general. We will give another argument based on Theorem 2.15 in a similar situation later (Proposition 3.8), we omit the detail here.

\section{Instantons on ALE spaces}

Quiver varieties were originally introduced by generalizing the ADHM description of instantons on ALE spaces by Kronheimer and the author [16]. In this section we go back to the original description and explain partial resolutions in terms of instantons (or sheaves) on (possibly singular) ALE spaces.

\subsection{ALE spaces}

We review Kronheimer's construction [15] of ALE spaces briefly in our terminology.

We consider the untwisted affine Lie algebra of type $A D E$. Let $0 \in I$ be the vertex corresponding to the simple root, which is the negative of the highest weight root of the corresponding simple Lie algebra. Let $I_{0} \stackrel{\text { def }}{=} I \backslash\{0\}$. Let $\delta$ be the vector in the kernel of the affine Cartan matrix whose 0 -component is equal to 1 . Such a vector is uniquely determined. Let $G_{\delta}$ be the complex Lie group corresponding to $\delta$ as in Section 2.1. Choose a parameter $\zeta^{\circ} \in \mathbb{R}^{I}$ from the level 0 hyperplane $\left\{\zeta \in \mathbb{R}^{I} \mid \zeta \cdot \delta=0\right\}$. Let us denote the corresponding quiver variety $\mathfrak{M}_{\zeta^{\circ}}(\delta, 0)$ for the parameter $\zeta^{\circ}$ by $X_{\zeta^{\circ}}$. This space is called an ALE space in the literature. We have a morphism

$$
\pi_{0, \zeta^{\circ}}: X_{\zeta^{\circ}} \rightarrow X_{0}
$$

from the construction in Section 2.5.

If we take $\zeta^{\circ}$ from an open face $F$ in the level 0 hyperplane, i.e., it is not contained in any real root hyperplane $D_{\theta}$, then $X_{\zeta^{\circ}}$ is nonsingular by Remark 2.13. Kronheimer [15] showed

(a) $X_{0}$ is isomorphic to $\mathbb{C}^{2} / \Gamma$, where $\Gamma$ is the finite subgroup of $\mathrm{SL}_{2}(\mathbb{C})$ associated to the affine Dynkin graph,

(b) (3.1) is the minimal resolution of $\mathbb{C}^{2} / \Gamma$, if $\zeta^{\circ}$ is taken as above.

For a later purpose we take a specific face $F^{\circ}$ with the above property and a parameter $\zeta^{\circ}$ as

$$
F^{\circ} \stackrel{\text { def }}{=}\left\{\zeta^{\circ} \in \mathbb{R}^{I} \mid \zeta^{\circ} \cdot \delta=0 \text { and } \zeta_{i}^{\circ}>0 \text { for } i \neq 0\right\}
$$

By the Weyl group action, any open face in the level 0 hyperplane is mapped to $F^{\circ}$.

A face $F^{\bullet}$ in the closure of $F^{\circ}$ is of the form

$$
F^{\bullet}=\left\{\zeta^{\bullet} \in \mathbb{R}^{I} \mid \zeta^{\bullet} \cdot \delta=0, \zeta_{i}^{\bullet}=0(\text { resp. }>0) \text { for } i \in I_{0}^{0}\left(\text { resp. } I_{0}^{+}\right)\right\}
$$


for a disjoint decomposition $I_{0}=I_{0}^{0} \sqcup I_{0}^{+}$. We allow the cases $I_{0}^{0}=\varnothing$, i.e., $\zeta^{\bullet}=\zeta^{\circ}$ or $I_{0}^{0}=I_{0}$, i.e., $\zeta^{\bullet}=0$. From the construction in Section 2.5 we have

$$
X_{\zeta^{\circ}} \stackrel{\pi_{\zeta}, \zeta^{\circ}}{\longrightarrow} X_{\zeta} \stackrel{\pi_{0, \zeta}}{\longrightarrow} X_{0} \cong \mathbb{C}^{2} / \Gamma
$$

Kronheimer also showed that (see [15, Lemma 3.3])

(c) $X_{\zeta} \bullet$ is a partial resolution of $\mathbb{C}^{2} / \Gamma$ having singularities of type $\mathbb{C}^{2} / \Gamma^{\prime}$ for some different $\Gamma^{\prime} \subset \mathrm{SL}_{2}(\mathbb{C})$.

In fact, we can describe singularities of $X_{\zeta}$ explicitly. Recall that the exceptional set of the minimal resolution (3.1) consists of an union of the complex projective line. By [23] each irreducible component $C_{i}$ naturally corresponds to a nonzero vertex $i \in I_{0}$ as follows: $C_{i}$ consists of data $B$ having a quotient isomorphic to $S_{i}$, a module with $\mathbb{C}$ on the vertex $i$, and 0 on the other vertices. It is clear that $B$ is $\zeta^{\bullet}$-semistable, but not $\zeta^{\bullet}$-stable. Then the curves $C_{i}$ with $i \in I_{0}^{0}$ are contracted under the morphism $X_{\zeta^{\circ}} \stackrel{\pi_{\zeta^{\bullet}, \zeta^{\circ}}}{\longrightarrow} X_{\zeta}$ •

In order to show that $\pi_{\zeta}, \zeta^{\circ}$ does not contract further curves, we give the following more precise classification:

Lemma 3.2. $A \zeta^{\bullet}$-stable point $B \in \mu^{-1}(0) \subset \mathbf{M}(\mathbf{v}, 0)$ satisfying the normalization condition $\zeta^{\bullet} \cdot \mathbf{v}=0$ is of one of the following three forms:

(a) a point in $X_{\zeta^{\circ}} \backslash \bigcup_{i \in I_{0}^{0}} C_{i}$,

(b) $\mathbf{v}$ is a coordinate vector $\mathbf{e}_{i}$ for an $i \in I_{0}^{0}$, or

(c) $\mathbf{v}$ is $\delta-\alpha_{h}$, where $\alpha_{h}$ is the highest root vector of a connected component of the sub-Dynkin diagram $I_{0}^{0}$.

In cases (b), (c) the corresponding $\zeta^{\bullet}$-stable point is unique up to isomorphisms.

Let $\mathcal{C}$ be the set of components of the sub-Dynkin diagram $I_{0}^{0}$. Let $B_{c}$ denote (the isomorphism class of) a $\zeta^{\bullet}$-stable representation corresponding to a component $c \in \mathcal{C}$ in (c). Then the $S$ equivalence class $x_{c}$ of $B_{c} \oplus \bigoplus_{i \in c} S_{i}^{\oplus\left(\alpha_{h}\right)_{i}}$ defines a point in $X_{\zeta}$, where $\left(\alpha_{h}\right)_{i}$ is the $i^{\text {th }}$-entry of $\alpha_{h}$. If $c$ is a Dynkin diagram of type $A D E, X_{\zeta}$ has a singularity of the corresponding type around $x_{c}$. This is an example of the description in Section 2.7. The new graph has vertices $\widehat{I}=c \sqcup\{0\}$ with the Cartan matrix $\widehat{c}_{i j}=c_{i j}$ if $i, j \in c, \widehat{c}_{0 i}=-{ }^{t}\left(\delta-\alpha_{h}\right) \mathbf{C e}_{i}={ }^{t} \alpha_{h} \mathbf{C e}_{i}$ if $i \in c$, and $\widehat{c}_{00}=2$. This is a graph of affine type.

Proof of Lemma 3.2. Consider the criterion in Theorem 2.15. Since our graph is of affine type, we have $p(x)=1$ if $x$ is an imaginary root (i.e., $x=m \delta$ with $m \geq 1$ ) and $p(x)=0$ if $x$ is a real root. Therefore if $\mathbf{v}$ is imaginary root, the condition is equivalent to $\mathbf{v}=\delta$. This is the case (a). If $\mathbf{v}$ is a real root, then the condition is that $\mathbf{v}$ cannot have a nontrivial decomposition $\mathbf{v}=\sum_{t} \beta^{(t)}$ with $\zeta^{\bullet} \cdot \beta^{(t)}=0$. Then it is clear that we have either (b) or (c).

Next show that a $\zeta^{\bullet}$-stable point is unique in case (b), (c). It is clear in the case (b). Consider the case (c). We apply an argument used by Mukai for the case of rigid sheaves on a $K 3$ surface: Let $B, B^{\prime}$ be $\zeta^{\bullet}$-stable points with the underlying $I$-graded vector space $V$ of dimension vector $\delta-\alpha_{h}$. We consider the complex

$$
\begin{aligned}
& \mathrm{L}(V, V) \stackrel{\alpha}{\rightarrow} \mathrm{E}(V, V) \stackrel{\beta}{\rightarrow} \mathrm{L}(V, V), \\
& \alpha(\xi)=B \xi-\xi B^{\prime}, \quad \beta(C)=\varepsilon\left(B C-C B^{\prime}\right) .
\end{aligned}
$$

Since the alternating sum of dimensions is 2 , we cannot have both $\alpha$ is injective and $\beta$ is surjective. If $\alpha$ is not injective, we have a nonzero homomorphism $\xi$ from the module $B$ to $B^{\prime}$. 
Then considering submodules $\operatorname{Ker} \xi \subset B$ and $\operatorname{Im} \xi \subset B^{\prime}$, the $\zeta^{\bullet}$-stability of $B$ and $B^{\prime}$ imply $\operatorname{Ker} \xi=0$ and $\operatorname{Im} \xi=B^{\prime}$. Therefore $B$ and $B^{\prime}$ are isomorphic. If $\beta$ is not surjective, we consider $\beta^{*} \in \mathrm{L}\left(V^{*}, V^{*}\right) \cong \mathrm{L}(V, V)$ instead, we get the same assertion.

Finally consider the case $\mathbf{v}=\delta$. By Lemma $2.12(3 \mathrm{~b})$ and the subsequent remark $B$ is $\zeta^{\circ}$ stable. So $B$ corresponds to a point $[B]$ in $X_{\zeta^{\circ}}$. Therefore it only remains to determine which point in $X_{\zeta^{\circ}}$ is $\zeta^{\bullet}$-stable.

If $B$ is not $\zeta^{\bullet}$-stable, we consider a Jordan-Hölder filtration to find a proper quotient which is $\zeta^{\bullet}$-stable. By the above discussion, the quotient must have the dimension vector $\mathbf{e}_{i}$ with $i \in I_{0}^{0}$ or $\delta-\alpha_{h}$. But in the latter case, $B$ contains a submodule with the dimension vector $\mathbf{e}_{i}$ with $i \in I_{0}^{0}$. This is impossible for the $\zeta^{\circ}$-stability, as $\zeta^{\circ} \cdot \mathbf{e}_{i}>0$. Thus $B$ has the quotient with the dimension vector $\mathbf{e}_{i}$ with $i \in I_{0}^{0}$. In particular, $\beta$ above is not surjective, and hence $B$ is contained in the exceptional component $C_{i}$ by [23, Proof of 5.10]. On the other hand, if $B$ is in $C_{i}, \beta$ is not surjective, and it has a quotient with $\operatorname{dim}=\mathbf{e}_{i}$. It is not $\zeta^{\bullet}$-stable. Therefore $\zeta^{\bullet}$-stable points are precisely in $X_{\zeta^{\circ}} \backslash \bigcup_{i \in I_{0}^{0}} C_{i}$.

Remark 3.3. It is not so difficult to prove the criterion in Theorem 2.15 directly without referring to a general result [8] in this particular example. The detail is left for a reader as an exercise.

\subsection{Sheaves and instantons on ALE spaces}

Let $\zeta^{\circ}, \zeta^{\bullet}$ be as in the previous subsection. We consider the corresponding quiver varieties $\mathfrak{M}_{\zeta^{\circ}}(\mathbf{v}, \mathbf{w}), \mathfrak{M}_{\zeta^{\bullet}}(\mathbf{v}, \mathbf{w})$ for $\mathbf{w} \neq 0$. By the main result of $[16]$, the former space $\mathfrak{M}_{\zeta^{\circ}}(\mathbf{v}, \mathbf{w})$ is the Uhlenbeck (partial) compactification of framed instantons on $X_{\zeta^{\circ}}$. Since we do not need to recall what instantons or their framing mean in this paper, we refer the definitions to the original paper [16]. Rather we use a different (but closely related) description in [30]. We first compactify $X_{\zeta^{\circ}}$ to an orbifold $\bar{X}_{\zeta^{\circ}}$ by adding $\ell_{\infty} \stackrel{\text { def }}{=} \mathbb{P}^{1} / \Gamma$. It is obtained by resolving the (isolated) singularity at 0 of $\mathbb{P}^{2} / \Gamma$. Then $\mathfrak{M}_{\zeta^{\circ}}^{\mathrm{s}}(\mathbf{v}, \mathbf{w})$ is a fine moduli space of framed holomorphic orbifold vector bundles $(E, \Phi)$, where $\Phi$ is an isomorphism between $\left.E\right|_{\ell_{\infty}}$ and $\left(\rho \otimes \mathcal{O}_{\mathbb{P}^{1}}\right) / \Gamma$ for a fixed representation $\rho$ of $\Gamma$. Here $\rho$ corresponds to the vector $\mathbf{w}$ via the McKay correspondence: $w_{i}$ is the multiplicity of the irreducible representation $\rho_{i}$ in $\rho$. And $\mathbf{v}$ corresponds to Chern classes of $E$. Since the explicit formula is not relevant here, we refer to $[30,(1.9)]$ for an interested reader.

Let us consider $\mathfrak{M}_{\zeta^{\circ}}(\mathbf{v}, \mathbf{w})$ which is a partial compactification of $\mathfrak{M}_{\zeta^{\circ}}^{\mathrm{s}}(\mathbf{v}, \mathbf{w})$. Its closed point is represented by an $S$-equivalence class of $\zeta^{\circ}$-semistable points, and hence a direct sum of $\zeta^{\circ}$ stable point all having $\theta_{\zeta^{\circ}}=0$. By [16, Proposition 9.2(ii)] (or [30, 4.3] for a detailed argument) such a point is of a form $(B, a, b)=\left(B^{\prime}, a^{\prime}, b^{\prime}\right) \oplus x_{1} \oplus x_{2} \oplus \cdots \oplus x_{k}$, where $\left(B^{\prime}, a^{\prime}, b^{\prime}\right) \in \mathfrak{M}_{\zeta^{\circ}}^{\mathrm{s}}\left(\mathbf{v}^{0}, \mathbf{w}\right)$ with $\mathbf{w} \neq 0$ and $x_{i} \in \mathfrak{M}_{\zeta^{\circ}}(\delta, 0)$ corresponds to a point in the ALE space $X_{\zeta^{\circ}}$. It is also a special case of Lemma 3.2 with $I_{0}^{0}=\varnothing$. Therefore $\mathfrak{M}_{\zeta^{\circ}}(\mathbf{v}, \mathbf{w})$ is written as

$$
\mathfrak{M}_{\zeta^{\circ}}(\mathbf{v}, \mathbf{w})=\bigsqcup_{k \geq 0} \mathfrak{M}_{\zeta^{\circ}}^{\mathrm{s}}(\mathbf{v}-k \delta, \mathbf{w}) \times S^{k} X_{\zeta^{\circ}}
$$

where $S^{k} X_{\zeta^{\circ}}$ is the $k^{\text {th }}$ symmetric product of $X_{\zeta^{\circ}}$. As $\mathfrak{M}_{\zeta^{\circ}}^{\mathrm{s}}(\mathbf{v}-k \delta, \mathbf{w})$ is the framed moduli space of orbifold holomorphic vector bundles on $\bar{X}_{\zeta^{\circ}}$ with a smaller second Chern number, the above description means that $\mathfrak{M}_{\zeta^{\circ}}(\mathbf{v}, \mathbf{w})$ is the Uhlenbeck (partial) compactification of $\mathfrak{M}_{\zeta^{\circ}}^{\mathrm{s}}(\mathbf{v}, \mathbf{w})$.

Let us apply the results in Section 2.7 in this situation. In order to give a decomposition (2.17) we need to introduce a finer stratification for the symmetric power:

$$
S^{k} X_{\zeta^{\circ}}=\bigsqcup_{|\lambda|=k} S_{\lambda}^{k} X_{\zeta^{\circ}}
$$


where $\lambda=\left(\lambda_{1} \geq \lambda_{2} \geq \cdots \geq \lambda_{l}>0\right)$ is a partition of $k$ and $S_{\lambda}^{k} X_{\zeta^{\circ}}$ consists of those configurations of form $\sum_{n=1}^{l} \lambda_{n}\left[x_{n}\right]$ for $x_{n}$ distinct. If we take a module from a stratum $\mathfrak{M}_{\zeta^{\circ}}^{\mathrm{s}}(\mathbf{v}-k \delta, \mathbf{w}) \times S_{\lambda}^{k} X_{\zeta^{\circ}}$, the corresponding graph is $\widehat{I}=\{1, \ldots, l=l(\lambda)\}$, with $\widehat{c}_{k k^{\prime}}=0$. This is the disjoint union of $l=l(\lambda)$ copies of the edge loop. We have $\hat{W}_{k}=\mathbb{C}^{t} \mathbf{w} \delta$. Therefore $\mathfrak{M}_{\zeta^{\circ}}(\mathbf{v}, \mathbf{w})$ is locally isomorphic to $\mathbb{C}^{\operatorname{dim} \mathfrak{M}_{\zeta^{\circ}}^{s}(\mathbf{v}-k \delta, \mathbf{w})} \times \prod_{k=1}^{l} M_{0}\left(\mathbb{C}^{\lambda_{k}}, \mathbb{C}^{t} \mathbf{w} \delta\right)$, where $M_{0}($,$) is the quiver variety associated with$ the Jordan quiver, a single vertex and a single edge loop, which is known to be the Uhlenbeck partial compactification for $\mathbb{C}^{2}[25]$.

Next consider the stability parameter $\zeta^{\bullet}$. By Lemma 3.2 we have

$$
\mathfrak{M}_{\zeta^{\bullet}}(\mathbf{v}, \mathbf{w})=\bigsqcup \mathfrak{M}_{\zeta^{\bullet}}^{\mathrm{s}}\left(\mathbf{v}^{0}, \mathbf{w}\right) \times S_{\lambda}^{|\lambda|}\left[X_{\zeta^{\circ}} \backslash \bigcup_{i \in I_{0}^{0}} C_{i}\right] \times\left\{\bigoplus_{c \in \mathcal{C}}\left(B_{c}^{\oplus n_{c}} \oplus \bigoplus_{i \in c} S_{i}^{\oplus m_{i}}\right)\right\}
$$

where $c$ runs over the set $\mathcal{C}$ of connected components of the sub-Dynkin diagram $I_{0}^{0}$ with the highest root $\alpha_{h}^{c}$, and $B_{c}$ is a $\zeta^{\bullet}$-stable module with the dimension vector $\delta-\alpha_{h}^{c}$, which is unique up to isomorphisms. (See Lemma 3.2.) We have an obvious constraint $\mathbf{v}=\mathbf{v}^{0}+|\lambda| \delta+$ $\sum_{i \in I_{0}^{0}} m_{i} \mathbf{e}_{i}+\sum_{c} n_{c}\left(\delta-\alpha_{h}^{c}\right)$.

This space can be considered as the partial Uhlenbeck compactification of the framed moduli space of instantons on the orbifold $X_{\zeta}$ by a simple generalization of the result in [16]. The factor $\mathfrak{M}_{\zeta}^{\mathrm{s}}\left(\mathbf{v}^{0}, \mathbf{w}\right)$ parametrizes holomorphic orbifold vector bundles (i.e., reflexive sheaves), and $S^{k}\left[X_{\zeta^{\circ}} \backslash \bigcup_{i \in I_{0}^{0}} C_{i}\right]$ are unordered points with multiplicities. These are usual factors appearing in the Uhlenbeck compactification. The second factor is the length of a 0-dimensional sheaf $Q$ supported on $X_{\zeta^{\circ}} \backslash \bigcup_{i \in I_{0}^{0}} C_{i}$. The last factor $B_{c}^{\oplus n_{c}} \oplus \bigoplus_{i \in c} S_{i}^{\oplus m_{i}}$ is new, and corresponds to a representation of the local fundamental group around the singular point $x_{c}$, which is a finite subgroup $\Gamma_{c}$ corresponding to the sub-Dynkin diagram $c$ via the McKay correspondence, i.e., $i \in c$ corresponds to a nontrivial irreducible representation, and $B_{c}$ corresponds to the trivial representation, which usually corresponds to the 0 -vertex. It corresponds to a 0 -dimensional sheaf $Q$ supported at $x_{c}$, but we encode not only its length, but also the $\Gamma_{c}$-module structure.

From the argument in [30] (after modified to the case of $X_{\zeta^{\bullet}}$ ), we see that the morphism $\pi_{\zeta^{\bullet}, \zeta^{\circ}}$ is given in each stratum of (3.4) by

$$
\begin{aligned}
& \mathfrak{M}_{\zeta^{\circ}}^{\mathrm{s}}\left(\mathbf{v}^{0}, \mathbf{w}\right) \ni(E, \Phi) \mapsto\left(\left(\pi_{\zeta^{\bullet}, \zeta^{\circ}}\right)_{*}(E)^{\vee \vee}, \Phi\right) \in \mathfrak{M}_{\zeta^{\bullet}}^{\mathrm{s}}\left(\left(\mathbf{v}^{0}\right)^{\prime}, \mathbf{w}\right), \\
& S^{k} X_{\zeta^{\bullet}} \ni \sum \lambda_{n}\left[x_{n}\right] \mapsto \sum_{\xi_{n} \notin \cup C_{i}} \lambda_{n}\left[\pi_{\zeta^{\bullet}, \zeta^{\circ}}\left(x_{n}\right)\right],
\end{aligned}
$$

and the remaining factor $\bigoplus_{c \in \mathcal{C}}\left(B_{c}^{\oplus n_{c}} \oplus \bigoplus_{i \in c} S_{i}^{\oplus m_{i}}\right)$ is determined so that the map preserve the dimension vector $\mathbf{v}$.

Let us apply the local description in Section 2.7 in this situation. Take a point $x$ from the stratum of the above form. Then the graph $\widehat{I}$ is the disjoint union of $l=l(\lambda)$ copies of Jordan quivers and the affine graphs corresponding to the connected components $c$ of $\mathcal{C}$ (i.e., we add the 0 -vertex to $c$ ). The dimension vectors $\widehat{\mathbf{v}}, \widehat{\mathbf{w}}$ have $\lambda_{i},{ }^{t} \mathbf{w} \delta$ in the components for Jordan quivers. The components for the affine graph attached to $c$ are

$$
\widehat{\mathbf{v}}:\left(n_{c},\left(m_{i}\right)_{i \in c}\right), \quad \widehat{\mathbf{w}}:\left({ }^{t}\left(\delta-\alpha_{h}^{c}\right)\left(\mathbf{w}-\mathbf{C v}^{0}\right),\left.\left(\mathbf{w}-\mathbf{C v}^{0}\right)\right|_{c}\right),
$$

where the first components are the entries for the 0-vertex, and $\left.()\right|_{c}$ means taking the components in $c$. In particular the entries of $\widehat{\mathbf{w}}$ must be nonnegative. This follows from the same argument as in the proof of Proposition 2.25(2) and Lemma 2.28. We consider the complex $\mathscr{C}_{k l}$ in Section 2.7 for $[B, a, b] \in \mathfrak{M}_{\zeta}^{\mathrm{s}} \cdot\left(\mathbf{v}^{0}, \mathbf{w}\right)$ and $B_{c}, S_{i}$.

Following [30] we take the chamber $\mathcal{C}$ containing $\zeta$ with

$$
\zeta_{i}=\zeta_{i}^{\circ} \text { for } i \neq 0, \quad \zeta \cdot \delta \text { is a sufficiently small negative number. }
$$


Then $\mathcal{C}$ contains $\zeta^{\circ}$ in its closure. Therefore we have a morphism $\pi_{\zeta^{\circ}, \zeta}: \mathfrak{M}_{\zeta} \rightarrow \mathfrak{M}_{\zeta^{\circ}}$. The main result of [30] says that $\mathfrak{M}_{\zeta}$ is a fine moduli space of framed torsion free sheaves $(E, \Phi)$, where $\Phi$ is as above. (In particular, $E$ is locally free on $\ell_{\infty}$.) By its proof the morphism $\pi_{\zeta^{\circ}, \zeta}$ is given by the association

$$
(E, \Phi) \mapsto\left(\left(E^{\vee \vee}, \Phi\right), \operatorname{len}\left(E^{\vee \vee} / E\right)\right),
$$

where $E^{\vee \vee}$ is the double dual of $E$, which is locally free as $X_{\zeta^{\circ}}$ is a nonsingular surface, and $\operatorname{len}\left(E^{\vee \vee} / E\right)$ is the length of $E^{\vee \vee} / E$ considered as a configuration of unordered points in $X_{\zeta^{\circ}}$ counted with multiplicities. This $\mathfrak{M}_{\zeta}$ is called the Gieseker partial compactification of the framed moduli $\mathfrak{M}_{\zeta^{\circ}}^{\mathrm{s}}$ of locally free sheaves on the ALE space $X_{\zeta^{\circ}}$.

Strictly speaking we cannot take $\zeta$ independently from $\mathbf{v}$. When $\mathbf{v}$ becomes larger, we need to take $\zeta$ closer and closer to $\zeta^{\circ}$. In particular, we cannot specify $\zeta$ when we move $\mathbf{v}$ (as we will do in Section 5). Since it is cumbersome to use different notation for $\zeta$ for each $\mathbf{v}$, we simply denote all parameters by $\zeta$.

In summary we have four spaces and morphisms between them:

$$
\mathfrak{M}_{\zeta} \stackrel{\pi_{\zeta^{\circ}, \zeta}}{\longrightarrow} \mathfrak{M}_{\zeta^{\circ}} \stackrel{\pi_{\zeta}, \zeta^{\circ}}{\longrightarrow} \mathfrak{M}_{\zeta} \stackrel{\pi_{0, \zeta}}{\longrightarrow} \mathfrak{M}_{0},
$$

where $\mathfrak{M}_{\zeta}$ is the Gieseker partial compactification on $X_{\zeta^{\circ}}$, and $\mathfrak{M}_{\zeta^{\circ}}, \mathfrak{M}_{\zeta^{\bullet}}, \mathfrak{M}_{0}$ are the Uhlenbeck partial compactification on $X_{\zeta^{\circ}}, X_{\zeta}, X_{0}=\mathbb{C}^{2} / \Gamma$ respectively.

Proposition 3.8 (cf. $[24, \mathbf{1 0 . 5}, \mathbf{1 0 . 8}])$. (1) Suppose ${ }^{t} \delta \mathbf{w}=1$. Then $\mathfrak{M}_{\zeta}^{\mathrm{s}}(\mathbf{v}, \mathbf{w}) \neq \varnothing$ if and only if $\mathbf{w}-\mathbf{v}$ is in the Weyl group orbit of $\mathbf{w}$ and satisfies

$$
{ }^{t}\left(\delta-\alpha_{h}^{c}\right)(\mathbf{w}-\mathbf{C v}) \geq 0 \quad \text { for } c \in \mathcal{C}, \quad{ }^{t} \mathbf{e}_{i}(\mathbf{w}-\mathbf{C v}) \geq 0 \quad \text { for } i \in I_{0}^{0} .
$$

(2) Suppose ${ }^{t} \delta \mathbf{w} \geq 2$. Then $\mathfrak{M}_{\zeta}^{\mathrm{s}}(\mathbf{v}, \mathbf{w}) \neq \varnothing$ if and only if $\mathbf{w}-\mathbf{v}$ is a weight of the irreducible integrable highest weight module $V(\mathbf{w})$ and the above inequalities hold.

Proof. One can give a proof along arguments in [24, 10.5, 10.8], but we use Theorem 2.15 here.

If $\mathfrak{M}_{\zeta}^{\mathrm{s}}(\mathbf{v}, \mathbf{w}) \neq \varnothing$, then $\mathbf{w}-\mathbf{v}$ is a weight of the irreducible integrable highest weight module by Theorem 2.15(2). Moreover two inequalities hold, as we have explained why $\widehat{\mathbf{w}}$ has nonnegative entries. If ${ }^{t} \delta \mathbf{w}=1$, the second proof of Proposition 2.30 shows $\mathbf{w}-\mathbf{v} \in W \cdot \mathbf{w}$ is also necessary.

For the converse, we check the criterion in Theorem 2.15. From our choice of $\zeta^{\bullet}$, a positive root $\beta^{(t)}$ with $\zeta^{\bullet} \cdot \beta^{(t)}=0$ is one of the following:

(a) $m \delta$ for $m>0$,

(b) $m \delta+\alpha$ for $m \geq 0, \alpha \in \Delta\left(I_{0}^{0}\right)_{+}$, or

(c) $m \delta-\alpha$ for $m>0, \alpha \in \Delta\left(I_{0}^{0}\right)_{+}$,

where $\Delta\left(I_{0}^{0}\right)_{+}$is the set of positive roots of the subroot system $I_{0}^{0}$. We have

$$
{ }^{t}(m \delta)(\mathbf{w}-\mathbf{C v})=m^{t} \delta \mathbf{w} \geq 1, \quad{ }^{t}(m \delta+\alpha)(\mathbf{w}-\mathbf{C v}) \geq 0, \quad{ }^{t}(m \delta-\alpha)(\mathbf{w}-\mathbf{C v}) \geq 0
$$

from the assumption. Then

$$
\begin{aligned}
{ }^{t} \mathbf{v}(\mathbf{w} & \left.-\frac{1}{2} \mathbf{C v}\right)-{ }^{t} \mathbf{v}^{0}\left(\mathbf{w}-\frac{1}{2} \mathbf{C} \mathbf{v}^{0}\right)-\sum p\left(\beta^{(t)}\right) \\
& =\sum_{t}{ }^{t} \beta^{(t)}(\mathbf{w}-\mathbf{C v})-\#\left\{t \mid \beta^{(t)} \in \mathbb{Z}_{>0} \delta\right\}+\frac{1}{2}\left(\sum{ }^{t} \beta^{(t)}\right) \mathbf{C}\left(\sum \beta^{(t)}\right)
\end{aligned}
$$

is nonnegative. Suppose that this is 0 . Then we must have ${ }^{t} \delta \mathbf{w}=1$ and all $\beta^{(t)}$ are $\delta$. This case is excluded in (1) from the assumption that $\mathbf{w}-\mathbf{v}$ is the Weyl group orbit of $\mathbf{w}$. (See the proof of Proposition 2.30.) 


\section{Crystal and the branching}

In this section ${ }^{2}$, we assume the graph has no edge loops. Then it corresponds to a symmetric Kac-Moody Lie algebra $\mathfrak{g}$. Let $\zeta, \zeta^{\bullet}$ as in Section 2.8. We have the decomposition $I=I^{0} \sqcup I^{+}$. We have the Levi subalgebra $\mathfrak{g}_{I^{0}} \subset \mathfrak{g}$ corresponding to the subdiagram $I^{0}$. Take and fix $\mathbf{w} \neq 0$. This is identified with a dominant integral weight $\sum_{i} w_{i} \Lambda_{i}$ of $\mathfrak{g}$.

Let $\mathfrak{L}_{\zeta}(\mathbf{v}, \mathbf{w}) \subset \mathfrak{M}_{\zeta}(\mathbf{v}, \mathbf{w})$ be the subvariety $\pi_{0, \zeta}^{-1}(0)$. It is a Lagrangian subvariety $[22,5.8]$ if $\mathfrak{M}_{\zeta}(\mathbf{v}, \mathbf{w}) \neq \varnothing$. Let $\operatorname{Irr} \mathfrak{L}_{\zeta}(\mathbf{v}, \mathbf{w})$ be the set of irreducible components of $\mathfrak{L}_{\zeta}(\mathbf{v}, \mathbf{w})$ and let $\operatorname{Irr} \mathfrak{L}_{\zeta}(\mathbf{w})$ be their disjoint union $\bigsqcup_{\mathbf{v}} \operatorname{Irr} \mathfrak{L}_{\zeta}(\mathbf{v}, \mathbf{w})$. Kashiwara and Saito [13, 33], based on an earlier construction due to Lusztig [18], constructed a $\mathfrak{g}$-crystal structure on $\operatorname{Irr} \mathfrak{L}_{\zeta}(\mathbf{w})$ which is isomorphic to the crystal of the irreducible representation $V(\mathbf{w})$ of the quantum enveloping algebra $\mathbf{U}_{q}(\mathfrak{g})$ with the highest weight $\mathbf{w}$. (See [27] for a different proof.)

We do not recall the definition of the crystal and the construction in $[13,33]$ except two key ingredients, which are the weight and a function, usually denoted by $\varepsilon_{i}: \operatorname{If} Y \in \operatorname{Irr} \mathfrak{L}_{\zeta}(\mathbf{w})$, then $\mathrm{wt}(Y)=\mathbf{w}-\mathbf{v}$. We consider the complex $(2.27)$ for a generic element $(B, a, b)$ in $Y$. Then we have $\varepsilon_{i}(Y)=\operatorname{codim} \operatorname{Im} \beta_{i}$. Note also that $\left\langle h_{i}, \operatorname{wt}(Y)\right\rangle$ is the alternating sum of the dimensions of terms in (2.27), where the middle term contribute in + .

From a general theory on the crystal, the $\mathfrak{g}_{I^{0}}$-crystal of the restriction of $V(\mathbf{w})$ to the subalgebra $\mathbf{U}_{q}\left(\mathfrak{g}_{I^{0}}\right) \subset \mathbf{U}_{q}(\mathfrak{g})$ is given by forgetting $i$-arrows with $i \notin I^{0}$. Each connected component, which is isomorphic to the crystal of an irreducible highest weight representation of $\mathbf{U}_{q}\left(\mathfrak{g}_{I^{0}}\right)$ has the unique element $Y$ corresponding to the highest weight vector. It is characterized by the property $\varepsilon_{i}(Y)=0$ for any $i \in I^{0}$. We will give its geometric characterization.

Let $\mathfrak{L}_{\zeta^{\bullet}}^{\text {s }}(\mathbf{v}, \mathbf{w}) \stackrel{\text { def }}{=} \mathfrak{M}_{\zeta}^{\mathrm{s}}(\mathbf{v}, \mathbf{w}) \cap \pi_{0, \zeta}^{-1} \bullet(0)$. Since $\pi_{\zeta, \zeta}$ is an isomorphism on the preimage of $\mathfrak{M}_{\zeta}^{\mathrm{s}}(\mathbf{v}, \mathbf{w})$ (see Section 2.5), this can be identified with $\pi_{\zeta^{\bullet}, \zeta}^{-1}\left(\mathfrak{M}_{\zeta}^{\mathrm{s}} \bullet(\mathbf{v}, \mathbf{w})\right) \cap \mathfrak{L}_{\zeta}(\mathbf{v}, \mathbf{w})$. The latter is an open subvariety in $\mathfrak{L}_{\zeta}(\mathbf{v}, \mathbf{w})$. Hence $\mathfrak{L}_{\zeta}^{\mathbf{s}}(\mathbf{v}, \mathbf{w})$ is of pure dimension with $\operatorname{dim} \mathfrak{L}_{\zeta}^{\mathbf{s}}(\mathbf{v}, \mathbf{w})=$ $\operatorname{dim} \mathfrak{M}_{\zeta^{\bullet}}^{\mathrm{s}}(\mathbf{v}, \mathbf{w}) / 2$. Let $\operatorname{Irr} \mathfrak{L}_{\zeta}^{\mathrm{s}}(\mathbf{v}, \mathbf{w})$ be the set of irreducible components of $\mathfrak{L}_{\zeta^{\bullet}}^{\mathrm{s}}(\mathbf{v}, \mathbf{w})$. From what is explained above, this is a subset of $\operatorname{Irr} \mathfrak{L}_{\zeta}(\mathbf{v}, \mathbf{w})$ consisting of those $Y$ which intersect with the open subset $\pi_{\zeta^{\bullet}, \zeta}^{-1}\left(\mathfrak{M}_{\zeta}^{\mathrm{s}}(\mathbf{v}, \mathbf{w})\right)$.

Theorem 4.1. The set of irreducible components $Y \in \operatorname{Irr} \mathfrak{L}_{\zeta}(\mathbf{v}, \mathbf{w})$ with $\varepsilon_{i}(Y)=0$ for any $i \in I^{0}$ is identified with $\operatorname{Irr} \mathfrak{L}_{\zeta}^{\mathbf{s}} \bullet(\mathbf{v}, \mathbf{w})$.

Corollary 4.2. The multiplicity of the irreducible highest weight module $V_{I^{0}}\left(\mathbf{w}^{\prime}\right)$ of $\mathbf{U}_{q}\left(\mathfrak{g}_{I^{0}}\right)$ in the restriction of $V(\mathbf{w})$ is equal to the number of $Y \in \bigsqcup_{\mathbf{v}} \operatorname{Irr} \mathfrak{L}_{\zeta_{\bullet}}^{\mathbf{s}}(\mathbf{v}, \mathbf{w})$ such that the restriction of $\mathrm{wt}(Y)$ to $I^{0}$ is $\mathbf{w}^{\prime}$.

Note that the restriction of $\operatorname{wt}(Y)$ to $I^{0}$ is dominant for $Y \in \operatorname{Irr} \mathfrak{L}_{\zeta}^{\mathbf{s}}(\mathbf{v}, \mathbf{w})$ thanks to Lemma 2.28 .

The theorem follows from the following:

Lemma 4.3. Suppose that $x=[B, a, b] \in \mathfrak{M}_{\zeta}(V, W)$ is regular. Then $\operatorname{Im} \beta_{i}=V_{i}$ for all $i \in I^{0}$ if and only if $(B, a, b)$ is $\zeta^{\bullet}$-stable.

This was proved in [26, 2.9.4] (which was essentially a collection of arguments in [24]) in the special case $I=I^{0}$. The same proof works in general. We reproduce the proof for the sake of a reader.

Proof. The 'if' part follows from Lemma 2.28 as the case (a) is excluded as we assumed $W \neq 0$.

The 'only if' part: Since $(B, a, b)$ is $\zeta^{\bullet}$-semistable by Lemma 2.12 we take its Jordan-Hölder filtration in Theorem 2.8. We must have $k_{W}=N$, as the submodule $V^{k_{W}+1}$ violates the $\zeta$ stability otherwise. Therefore if $N \neq 0, \operatorname{gr}_{0}(B, a, b)$ has the $W$-component 0 , therefore it is

\footnotetext{
${ }^{2}$ As we mentioned in the introduction, the results of this section was already obtained by Malkin [19].
} 
0 -stable module for the subgraph $I^{0}$ extended to the whole graph by 0 . But the regularity assumption implies that it must be $S_{i}$ for $i \in I^{0}$. In particular, $\beta_{i}$ is not surjective.

Note that $Y \in \operatorname{Irr} \mathfrak{L}_{\zeta}(\mathbf{v}, \mathbf{w})$ is mapped into the closure of the stratum

$$
\mathfrak{M}_{\zeta^{\bullet}}^{\mathrm{s}}\left(\mathbf{v}^{\prime}, \mathbf{w}\right) \times\left\{\bigoplus_{i \in I^{0}} S_{i}^{\oplus \varepsilon_{i}(Y)}\right\} \quad \text { with } \quad \mathbf{v}^{\prime}=\mathbf{v}-\sum_{i \in I^{0}} \varepsilon_{i}(Y) \mathbf{e}_{i}
$$

by the morphism $\pi_{\zeta \bullet, \zeta}$.

\section{Convolution algebra and partial resolution}

We assume the graph has no edge loops and the stability parameters are either $\zeta$, $\zeta^{\bullet}$ as in Section 2.8 or as in Section 3.2 (and assume the graph is of an affine type).

\subsection{General results}

We fix $\mathbf{w}$ and consider unions of quiver varieties $\mathfrak{M}_{\zeta}(\mathbf{v}, \mathbf{w}), \mathfrak{M}_{\zeta}(\mathbf{v}, \mathbf{w}), \mathfrak{M}_{0}(\mathbf{v}, \mathbf{w})$ over various v's. For $\mathfrak{M}_{0}$ and $\mathfrak{M}_{\zeta}$, they were introduced in [26, Section 2.5]:

$$
\mathfrak{M}_{0}(\mathbf{w}) \stackrel{\text { def }}{=} \bigcup_{\mathbf{v}} \mathfrak{M}_{0}(\mathbf{v}, \mathbf{w}), \quad \mathfrak{M}_{\zeta}(\mathbf{w}) \stackrel{\text { def }}{=} \bigsqcup_{\mathbf{v}} \mathfrak{M}_{\zeta}(\mathbf{v}, \mathbf{w})
$$

where we take the union in $\mathfrak{M}_{0}(\mathbf{v}, \mathbf{w})$ with respect to the closed immersion $\mathfrak{M}_{0}(V, W) \subset \mathfrak{M}_{0}(V \oplus$ $V^{\prime}, \mathbf{w}$ ) induced by the extension by 0 to the component $V^{\prime}$. (In [26, Section 2.5] this was denoted by $\mathfrak{M}_{0}(\infty, \mathbf{w})$.) These are infinite unions of varieties (of various dimensions), but there is no difficulty as we can work on finitely many v's in any of later constructions.

For $\mathfrak{M}_{\zeta}$ we mimic this construction. For $\zeta \bullet$ in Section 2.8 we consider the closed immersion $\mathfrak{M}_{\zeta}(V, W) \subset \mathfrak{M}_{\zeta^{\bullet}}\left(V \oplus V^{\prime}, \mathbf{w}\right)$ with an $I^{0}$-graded (instead of $I$-graded) vector space $V^{\prime}$ and take the union. For $\zeta^{\bullet}$ in Section 3.2 we similarly consider $\mathfrak{M}_{\zeta}(V, W) \subset \mathfrak{M}_{\zeta}\left(V \oplus V^{\prime}, \mathbf{w}\right)$ where $V^{\prime}$ is a direct sum of various $S_{i}$ and $B_{c}$ 's in (3.5). This union is compatible with the morphism $\pi_{0, \zeta}$ in either cases. We have the induced morphism $\pi_{0, \zeta}: \mathfrak{M}_{\zeta} \bullet(\mathbf{w}) \rightarrow \mathfrak{M}_{0}(\mathbf{w})$. We also have $\pi_{\zeta, \zeta}: \mathfrak{M}_{\zeta}(\mathbf{w}) \rightarrow \mathfrak{M}_{\zeta}(\mathbf{w})$

Let $Z_{\zeta} \bullet \zeta(\mathbf{w}) \stackrel{\text { def }}{=} \mathfrak{M}_{\zeta}(\mathbf{w}) \times_{\mathfrak{M}_{\zeta} \bullet(\mathbf{w})} \mathfrak{M}_{\zeta}(\mathbf{w})$ and similarly for $Z_{0, \zeta}(\mathbf{w})$. These are union of various subvarieties $Z_{\zeta} \bullet \zeta\left(\mathbf{v}^{1}, \mathbf{v}^{2} ; \mathbf{w}\right)$ or $Z_{0, \zeta}\left(\mathbf{v}^{1}, \mathbf{v}^{2} ; \mathbf{w}\right)$ in $\mathfrak{M}_{\zeta}\left(\mathbf{v}^{1}, \mathbf{w}\right) \times \mathfrak{M}_{\zeta}\left(\mathbf{v}^{2}, \mathbf{w}\right)$, where the fiber product is defined over a space $\mathfrak{M}_{\zeta}(\mathbf{v}, \mathbf{w})$ or $\mathfrak{M}_{0}(\mathbf{v}, \mathbf{w})$ with some large $\mathbf{v}$ compared with $\mathbf{v}^{1}, \mathbf{v}^{2}$. By Proposition $2.23 Z_{0, \zeta}\left(\mathbf{v}^{1}, \mathbf{v}^{2} ; \mathbf{w}\right), Z_{\zeta} \bullet \zeta\left(\mathbf{v}^{1}, \mathbf{v}^{2} ; \mathbf{w}\right)$ are at most half dimensional in $\mathfrak{M}_{\zeta}\left(\mathbf{v}^{1}, \mathbf{w}\right) \times \mathfrak{M}_{\zeta}\left(\mathbf{v}^{2}, \mathbf{w}\right)$. Moreover $Z_{\zeta} \bullet \zeta\left(\mathbf{v}^{1}, \mathbf{v}^{2} ; \mathbf{w}\right)$ is a subvariety of $Z_{0, \zeta}\left(\mathbf{v}^{1}, \mathbf{v}^{2} ; \mathbf{w}\right)$. The latter space was introduced in [24] as an analog of the Steinberg variety appearing in a geometric construction of the Weyl group.

We consider the top degree Borel-Moore homology groups $H_{\text {top }}\left(Z_{\zeta} \bullet \zeta(\mathbf{w})\right)$ and $H_{\text {top }}\left(Z_{0, \zeta}(\mathbf{w})\right)$. More precisely the former is the subspace

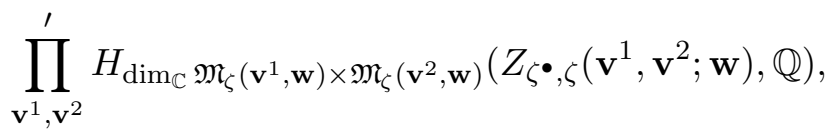

of the direct products consisting elements $\left(F_{\mathbf{v}, \mathbf{v}^{\prime}}\right)$ such that

1) for fixed $\mathbf{v}^{1}, F_{\mathbf{v}^{1}, \mathbf{v}^{2}}=0$ for all but finitely many choices of $\mathbf{v}^{2}$,

2) for fixed $\mathbf{v}^{2}, F_{\mathbf{v}^{1}, \mathbf{v}^{2}}=0$ for all but finitely many choices of $\mathbf{v}^{1}$.

Similarly for the latter. 
Since $\mathfrak{M}_{\zeta}(\mathbf{v}, \mathbf{w})$ is smooth, we have an associative algebra structure on $H_{\text {top }}\left(Z_{\zeta} \bullet \zeta(\mathbf{w})\right)$ and $H_{\text {top }}\left(Z_{0, \zeta}(\mathbf{w})\right)$ with the unit given by the sum of diagonals in $\mathfrak{M}_{\zeta}(\mathbf{v}, \mathbf{w}) \times \mathfrak{M}_{\zeta}(\mathbf{v}, \mathbf{w})$ for various $\mathbf{v}$. We have an injective algebra homomorphism

$$
H_{\text {top }}\left(Z_{\zeta}, \zeta(\mathbf{w})\right) \rightarrow H_{\text {top }}\left(Z_{0, \zeta}(\mathbf{w})\right)
$$

We can analyze irreducible representations of these algebras and the branching rules with respect to the above homomorphism by a general theory in [7] based on [3]. We prepare several notations and concepts, and then state the result.

For a point $x$ in a stratum $\mathfrak{M}_{0}(\mathbf{w})_{(\widehat{G})}$ of $\mathfrak{M}_{0}(\mathbf{w}) \operatorname{let} d_{x}(\mathbf{v}, \mathbf{w})=\operatorname{dim}_{\mathbb{C}} \mathfrak{M}_{\zeta}(\mathbf{v}, \mathbf{w})-\operatorname{dim}_{\mathbb{C}} \mathfrak{M}_{0}(\mathbf{w})_{(\widehat{G})}$ and $H_{\text {top }}\left(\pi_{0, \zeta}^{-1}(x)\right)$ be the direct sum of the cohomology groups of the fibers $\pi_{0, \zeta}^{-1}(x) \cap \mathfrak{M}_{\zeta}(\mathbf{v}, \mathbf{w})$ of the degree $d_{x}(\mathbf{v}, \mathbf{w})$. By the convolution product $H_{\text {top }}\left(\pi_{0, \zeta}^{-1}(x)\right)$ is a $H_{\text {top }}\left(Z_{0, \zeta}(\mathbf{w})\right)$-module. The cohomology vanishes in degree above $d_{x}(\mathbf{v}, \mathbf{w})$ and has a basis by $d_{x}(\mathbf{v}, \mathbf{w})$-dimensional irreducible components in degree $d_{x}(\mathbf{v}, \mathbf{w})$ by Proposition 2.23, and does not vanish in $d_{x}(\mathbf{v}, \mathbf{w})$ if the graph is of finite or affine type by Remark 2.24.

For a simple local system $\phi$ on $\mathfrak{M}_{0}(\mathbf{w})_{(\widehat{G})}$ (i.e., an irreducible representation of the fundamental group of $\left.\mathfrak{M}_{0}(\mathbf{w})_{(\widehat{G})}\right)$, let $I C\left(\mathfrak{M}_{0}(\mathbf{w})_{(\widehat{G})}, \phi\right)$ be the corresponding intersection cohomology complex.

The fundamental group of $\mathfrak{M}_{0}(\mathbf{w})_{(\widehat{G})}$ acts on $H_{\text {top }}\left(\pi_{0, \zeta}^{-1}(x)\right)$ by monodromy. It is a permutation of the above basis elements. We have a decomposition $H_{\text {top }}\left(\pi_{0, \zeta}^{-1}(x)\right)=\bigoplus \phi \otimes V_{(\widehat{G}), \phi}$ into the direct sum of simple local system $\phi$ tensored with the multiplicity vector space $V_{(\widehat{G}), \phi}$. A pair $\left(\mathfrak{M}_{0}(\mathbf{w})_{(\widehat{G})}, \phi\right)$ of a stratum $\mathfrak{M}_{0}(\mathbf{w})_{(\widehat{G})}$ and a simple local system $\phi$ on it is relevant for $\pi_{0, \zeta}$ if $V_{(\widehat{G}), \phi} \neq 0$. Then we have the decomposition theorem

$$
\left(\pi_{0, \zeta}\right)_{*}\left(\mathbb{C}_{\mathfrak{M}_{\zeta}(\mathbf{w})}[\operatorname{dim}]\right)=\bigoplus I C\left(\mathfrak{M}_{0}(\mathbf{w})_{(\widehat{G})}, \phi\right) \otimes V_{(\widehat{G}), \phi},
$$

where $\mathbb{C}_{\mathfrak{M}_{\zeta}(\mathbf{w})}[\operatorname{dim}]$ is the direct sum $\bigoplus_{\mathbf{v}} \mathbb{C}_{\mathfrak{M}_{\zeta}(\mathbf{v}, \mathbf{w})}\left[\operatorname{dim} \mathfrak{M}_{\zeta}(\mathbf{v}, \mathbf{w})\right]$. We also know $[7,8.9 .8]$ that

$$
H_{\text {top }}\left(Z_{0, \zeta}(\mathbf{w})\right) \cong \bigoplus \operatorname{End}_{\mathbb{C}}\left(V_{(\widehat{G}), \phi}\right) .
$$

Thus $\left\{V_{(\widehat{G}, \phi)} \mid\left(\left(\mathfrak{M}_{0}\right)_{(\widehat{G})}, \phi\right)\right.$ is relevant for $\left.\pi_{0, \zeta}\right\}$ is the set of isomorphism classes of irreducible representations of $H_{\text {top }}\left(Z_{0, \zeta}(\mathbf{w})\right)$.

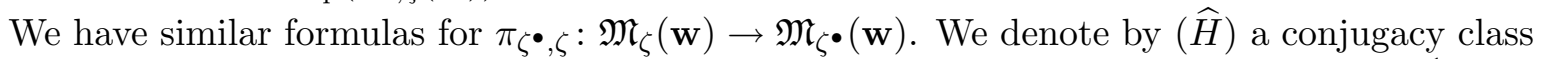
of $\mathrm{GL}(V)$ corresponding to a stratum of $\mathfrak{M}_{\zeta}(\mathbf{w})$. Similar to above we can define $H_{\text {top }}\left(\pi_{\zeta \bullet, \zeta}^{-1}(y)\right)$, which is a $H_{\text {top }}\left(Z_{\zeta}, \zeta(\mathbf{w})\right)$-module for $y \in \mathfrak{M}_{\zeta}(\mathbf{w})_{(\widehat{H})}$.

If we decompose the direct image of $\operatorname{IC}\left(\mathfrak{M}_{\zeta}(\mathbf{w})_{(\widehat{H})}, \psi\right)$ as

$$
\left(\pi_{0, \zeta}\right)_{*}\left(I C\left(\mathfrak{M}_{\zeta} \bullet(\mathbf{w})_{(\widehat{H})}, \psi\right)\right)=\bigoplus I C\left(\mathfrak{M}_{0}(\mathbf{w})_{(\widehat{G})}, \phi\right) \otimes V_{(\widehat{G}), \phi}^{(\widehat{H}), \psi}
$$

with the multiplicity vector space $V_{(\widehat{G}), \phi}^{(\widehat{H}),}$, we have the following branching rule from the double decomposition formula (see $[3,1.11]$ )

$$
\operatorname{Res} V_{(\widehat{G}), \phi}=\bigoplus V_{(\widehat{G}), \phi}^{(\widehat{H}), \psi} \otimes V_{(\widehat{H}), \psi},
$$

where Res is the restriction functor from $H_{\text {top }}\left(Z_{0, \zeta}(\mathbf{w})\right)$-modules to $H_{\text {top }}\left(Z_{\zeta, \zeta}(\mathbf{w})\right)$-modules via the injective homomorphism (5.1). Since $V_{(\widehat{H}), \psi}$ are pairwise non-isomorphic, $V_{(\widehat{G}), \phi}^{(\widehat{H}),}$ is the multiplicity space $\left.\operatorname{Hom}_{H_{\mathrm{top}}\left(Z_{\zeta} \bullet \zeta\right.}(\mathbf{w})\right)\left(V_{(\widehat{H}), \psi}\right.$, Res $\left.V_{(\widehat{G}), \phi}\right)$. 
Note also that $V_{(\widehat{G}), \phi}^{(\widehat{H}),}$ is the $\phi$-isotypical component of $H^{\operatorname{top}}\left(\pi_{0, \zeta}^{-1} \bullet(x), I C\left(\mathfrak{M}_{\zeta} \bullet(\mathbf{w})_{(\widehat{H})}, \psi\right)\right)\left[d_{y}\right]$ the top degree cohomology of $\pi_{0, \zeta}^{-1}(x)$ with the coefficient in the shifted IC sheaf, where $d_{y}=$ $\operatorname{dim} \mathfrak{M}_{\zeta}(\mathbf{w})-\operatorname{dim} \mathfrak{M}_{\zeta}(\mathbf{w})_{(\widehat{H})}$. (More precisely we consider the degree 'top' and $d_{y}$ for each $\mathbf{v}$ separately.) See $[3,1.10]$.

\subsection{The restriction to a Levi factor}

Let us consider the case when $\zeta, \zeta^{\bullet}$ are as in Section 2.8 in this subsection.

In [24] we constructed an algebra homomorphism

$$
\mathbf{U}(\mathfrak{g}) \rightarrow H_{\text {top }}\left(Z_{0, \zeta}(\mathbf{w})\right)
$$

The homomorphism was given on generators by

$$
e_{i} \mapsto \sum_{\mathbf{v}^{2}}\left[\mathfrak{P}\left(\mathbf{v}^{2}, \mathbf{w}\right)\right], \quad f_{i} \mapsto \sum_{\mathbf{v}^{2}} \pm\left[\omega \mathfrak{P}\left(\mathbf{v}^{2}, \mathbf{w}\right)\right], \quad h \mapsto \sum_{\mathbf{v}}\langle h, \mathbf{w}-\mathbf{v}\rangle[\Delta(\mathbf{v}, \mathbf{w})],
$$

where $\mathfrak{P}\left(\mathbf{v}^{2}, \mathbf{w}\right)$ is the 'Hecke correspondence' parametrizing pairs $\left(\left[B^{1}, a^{1}, b^{1}\right],\left[B^{2}, a^{2}, b^{2}\right]\right)$ such that $\left(B^{1}, a^{1}, b^{1}\right)$ is a submodule of $\left(B^{2}, a^{2}, b^{2}\right)$ with the quotient isomorphic to $S_{i}$, a module with $\mathbb{C}$ on the vertex $i$, and 0 on the other vertices, $\omega$ is the exchange of factors of $\mathfrak{M}_{\zeta}\left(\mathbf{v}^{1}, \mathbf{w}\right) \times$ $\mathfrak{M}_{\zeta}\left(\mathbf{v}^{2}, \mathbf{w}\right)$ and $\Delta(\mathbf{v}, \mathbf{w})$ is the diagonal in $\mathfrak{M}_{\zeta}(\mathbf{v}, \mathbf{w}) \times \mathfrak{M}_{\zeta}(\mathbf{v}, \mathbf{w})$. The \pm -sign in the definition of $f_{i}$ is not important in the discussion below, so its precise definition is omitted. From this definition it is clear that we have an algebra homomorphism

$$
\mathbf{U}\left(\mathfrak{g}_{I^{0}}+\mathfrak{h}\right) \rightarrow H_{\text {top }}\left(Z_{\zeta} \bullet \zeta(\mathbf{w})\right),
$$

where $\mathfrak{h}$ is the Cartan subalgebra of $\mathfrak{g}$ and $\mathfrak{g}_{I^{0}}$ is the Levi factor corresponding to the subset $I^{0} \subset I$.

We must be careful when we apply the result in the previous subsection, as this homomorphism is not an isomorphism.

Remark 5.5. Later we consider the case when $\mathfrak{g}$ is an affine Lie algebra. Our affine Lie algebra, as in [12], contains the degree operator $d$. It is mapped to $\sum_{\mathbf{v}}\langle d, \mathbf{w}-\mathbf{v}\rangle[\Delta(\mathbf{v}, \mathbf{w})]=$ $-\sum_{\mathbf{v}} v_{0}[\Delta(\mathbf{v}, \mathbf{w})]$, where 0 is the special vertex of the affine graph as in Section 3.1.

Let us denote by $\operatorname{IC}\left(\mathfrak{M}_{\zeta} \bullet(\mathbf{w})_{(\widehat{H})}\right)$ the IC sheaf corresponding to the trivial local system.

Theorem 5.6. Let $V^{\mathfrak{g}}(\lambda)$ (resp. $V^{\left(\mathfrak{g}_{I^{0}}+\mathfrak{h}\right)}(\lambda)$ ) denote the irreducible integrable highest weight module of $\mathfrak{g}\left(\right.$ resp. $\left.\mathfrak{g}_{I^{0}}+\mathfrak{h}\right)$ with the highest weight $\lambda$. Then we have

$$
\begin{aligned}
\left(\pi_{0, \zeta}\right)_{*}\left(I C\left(\mathfrak{M}_{\zeta}^{\mathrm{s}} \bullet\left(\mathbf{v}^{0}, \mathbf{w}\right)\right)\right)= & \bigoplus_{\mathbf{v}^{\prime}} \operatorname{Hom}_{\mathfrak{g}_{I^{0}}+\mathfrak{h}}\left(V^{\left(\mathfrak{g}_{I^{0}}+\mathfrak{h}\right)}\left(\mathbf{w}-\mathbf{v}^{0}\right), V^{\mathfrak{g}}\left(\mathbf{w}-\mathbf{v}^{\prime}\right)\right) \otimes I C\left(\mathfrak{M}_{0}^{\mathrm{s}}\left(\mathbf{v}^{\prime}, \mathbf{w}\right)\right) \\
& \bigoplus \bigoplus(I C \text { sheaves associated with non-regular strata })
\end{aligned}
$$

Proof. Let $\mathfrak{M}_{0}(\mathbf{w})_{(\widehat{G})}$ be a stratum of $\mathfrak{M}_{0}(\mathbf{w})$. Take a point $x \in \mathfrak{M}_{0}(\mathbf{w})_{(\widehat{G})}$. The decomposition $H_{\text {top }}\left(\pi_{0, \zeta}^{-1}(x)\right)=\bigoplus \phi \otimes V_{(\widehat{G}), \phi}$ in the previous subsection is a decomposition of a $\mathfrak{g}$-module. Suppose $\mathfrak{M}_{0}(\mathbf{w})_{(\widehat{G})}$ is regular, i.e., $=\mathfrak{M}_{0}^{\mathrm{s}}\left(\mathbf{v}^{\prime}, \mathbf{w}\right)$ for some $\mathbf{v}^{\prime}$. Then it is known that $H_{\text {top }}\left(\pi_{0, \zeta}^{-1}(x)\right)$ is an irreducible integrable representation of $\mathfrak{g}$ with the highest weight vector $[x] \in H_{\text {top }}\left(\pi_{0, \zeta}^{-1}(x) \cap\right.$ $\left.\mathfrak{M}_{\zeta}\left(\mathbf{v}^{\prime}, \mathbf{w}\right)\right)[24,10.2]$, where $x$ can be considered as a point in $\mathfrak{M}_{\zeta}\left(\mathbf{v}^{\prime}, \mathbf{w}\right)$ as $\pi_{0, \zeta}: \mathfrak{M}_{\zeta}\left(\mathbf{v}^{\prime}, \mathbf{w}\right) \rightarrow$ $\mathfrak{M}_{0}\left(\mathbf{v}^{\prime}, \mathbf{w}\right)$ is an isomorphism on the preimage of $\mathfrak{M}_{0}^{\mathrm{s}}\left(\mathbf{v}^{\prime}, \mathbf{w}\right)$. In particular, $V_{(\widehat{G}), \phi}=0$ unless $\phi$ is the trivial local system in this case. 
Similarly if a stratum $\mathfrak{M}_{\zeta^{\bullet}}(\mathbf{w})_{(\widehat{H})}$ is of the form $\mathfrak{M}_{\zeta^{\bullet}}^{\mathrm{s}}\left(\mathbf{v}^{0}, \mathbf{w}\right)$ for some $\mathbf{v}^{0}$, then $H_{\text {top }}\left(\pi_{\zeta^{\bullet}, \zeta}^{-1}(y)\right)$ is the irreducible integrable representation $V^{\left(\mathfrak{g}_{I^{0}}+\mathfrak{h}\right)}\left(\mathbf{w}-\mathbf{v}^{0}\right)$ if $y \in \mathfrak{M}_{\zeta}^{\mathrm{s}} \bullet\left(\mathbf{v}^{0}, \mathbf{w}\right)$. The highest weight vector is $[y]$ as above.

Consider a stratum $\mathfrak{M}_{\zeta^{\bullet}}(\mathbf{w})_{(\widehat{H})}$ which contains $I C\left(\mathfrak{M}_{0}^{\mathrm{s}}\left(\mathbf{v}^{\prime}, \mathbf{w}\right)\right)$ in the decomposition (5.3). We can write it as $\mathfrak{M}_{\zeta^{\bullet}}^{\mathbf{s}}\left(\mathbf{v}^{0}, \mathbf{w}\right) \times\left(\mathfrak{M}_{0}\right)_{\left(\widehat{H}^{\prime}\right)}$ as in (2.26). We consider the diagram (2.29). Since $\kappa$ is a finite birational morphism, we have

$$
I C\left(\overline{\mathfrak{M}_{\zeta^{\bullet}}^{\mathrm{s}}\left(\mathbf{v}^{0}, \mathbf{w}\right) \times\left(\mathfrak{M}_{0}\right)_{\left(\widehat{H}^{\prime}\right)}}\right)=\kappa_{*}\left(I C\left(\mathfrak{M}\left(\mathbf{v}^{0}, \mathbf{w}\right)\right) \otimes I C\left(\overline{\left(\mathfrak{M}_{0}\right)_{\left(\widehat{H}^{\prime}\right)}}\right)\right),
$$

and hence

$$
\left(\pi_{0, \zeta^{\bullet}}\right)_{*} I C\left(\overline{\mathfrak{M}_{\zeta}^{\mathrm{s}}\left(\mathbf{v}^{0}, \mathbf{w}\right) \times\left(\mathfrak{M}_{0}\right)_{\left(\widehat{H}^{\prime}\right)}}\right)=\kappa_{*}^{\prime}\left(\left(\pi_{0, \zeta}\right)_{*} I C\left(\mathfrak{M}\left(\mathbf{v}^{0}, \mathbf{w}\right)\right) \otimes I C\left(\overline{\left.\left(\mathfrak{M}_{0}\right)_{\left(\widehat{H}^{\prime}\right.}\right)}\right)\right)
$$

Since $\kappa^{\prime}$ is also a finite morphism, this contains only IC sheaves on nonregular strata unless the second factor $\left(\mathfrak{M}_{0}\right)_{\left(\widehat{H}^{\prime}\right)}$ is trivial. Hence (5.4) now becomes

$$
\operatorname{Res} V^{\mathfrak{g}}\left(\mathbf{w}-\mathbf{v}^{\prime}\right)=\bigoplus_{\mathbf{v}^{0}} V_{\mathbf{w}-\mathbf{v}^{\prime}}^{\mathbf{w}-\mathbf{v}^{0}} \otimes V^{\left(\mathfrak{g}_{I^{0}}+\mathfrak{h}\right)}\left(\mathbf{w}-\mathbf{v}^{0}\right)
$$

where $V_{\mathbf{w}-\mathbf{v}^{\prime}}^{\mathbf{w}-\mathbf{v}^{0}}$ is the multiplicity of $\operatorname{IC}\left(\mathfrak{M}_{0}^{\mathrm{s}}\left(\mathbf{v}^{\prime}, \mathbf{w}\right)\right)$ in the decomposition of $\left(\pi_{0, \zeta}\right)_{*}\left(\operatorname{IC}\left(\mathfrak{M}_{\zeta}^{\mathrm{s}} \bullet\left(\mathbf{v}^{0}\right.\right.\right.$, $\mathbf{w}))$ ) in (5.3). Thus $V_{\mathbf{w}-\mathbf{v}^{\prime}}^{\mathbf{w}-\mathbf{v}^{0}}=\operatorname{Hom}_{\mathfrak{g}_{I^{0}}+\mathfrak{h}}\left(V^{\left(\mathfrak{g}_{I^{0}}+\mathfrak{h}\right)}\left(\mathbf{w}-\mathbf{v}^{0}\right), V^{\mathfrak{g}}\left(\mathbf{w}-\mathbf{v}^{\prime}\right)\right)$. Note here that $V^{\left(\mathfrak{g}_{0^{0}}+\mathfrak{h}\right)}\left(\mathbf{w}-\mathbf{v}^{0}\right)$ are non-isomorphic for different $\mathbf{v}^{0}$.

We assume the graph is of affine type until the end of this subsection. This is because we know the structure of $H_{\text {top }}\left(\pi_{0, \zeta}^{-1}(x)\right)$ for a point $x$ from a nonregular stratum only in affine type. We further suppose $I^{0} \neq I$ to avoid the trivial situation $\pi_{0, \zeta}=$ id. We thus have

$$
\mathfrak{M}_{\zeta}(\mathbf{w})=\bigsqcup_{\mathbf{v}} \mathfrak{M}_{\zeta}^{\mathrm{s}}(\mathbf{v}, \mathbf{w})
$$

where $\mathbf{v}$ is such that $\mathbf{w}-\mathbf{v}$ is an $I^{0}$-dominant weight of $V(\mathbf{w})$.

Let us consider $\pi_{0, \zeta}: \mathfrak{M}_{\zeta}(\mathbf{w}) \rightarrow \mathfrak{M}_{0}(\mathbf{w})$. The stratification of $\mathfrak{M}_{0}(\mathbf{w})$ induced from (3.5) is

$$
\mathfrak{M}_{0}(\mathbf{w})=\bigsqcup \mathfrak{M}_{0}^{\mathrm{s}}\left(\mathbf{v}^{\prime}, \mathbf{w}\right) \times S_{\lambda}^{|\lambda|}\left(\mathbb{C}^{2} \backslash\{0\} / \Gamma\right)
$$

The criterion for $\mathfrak{M}_{0}^{\mathrm{s}}\left(\mathbf{v}^{\prime}, \mathbf{w}\right) \neq \varnothing$ was given in Proposition 2.30. Take a point $x$ from the stratum $\mathfrak{M}_{0}^{\mathrm{s}}\left(\mathbf{v}^{\prime}, \mathbf{w}\right) \times S_{\lambda}^{|\lambda|}\left(\mathbb{C}^{2} \backslash\{0\} / \Gamma\right)$ with $\lambda=\left(\lambda_{1}, \ldots, \lambda_{l}\right)(l=l(\lambda))$, and consider the inverse image under $\pi_{0, \zeta}: \mathfrak{M}_{\zeta}(\mathbf{v}, \mathbf{w}) \rightarrow \mathfrak{M}_{0}(\mathbf{v}, \mathbf{w})$. Then $\widehat{I}$ is the disjoint union of $I$ and $l$ copies of the Jordan quiver. The dimension vectors are

$$
\widehat{\mathbf{v}}=\left(\mathbf{v}-\mathbf{v}^{\prime}-|\lambda| \delta, \lambda_{1}, \ldots, \lambda_{l}\right), \quad \widehat{\mathbf{w}}=\left(\mathbf{w}-\mathbf{C v}^{\prime},{ }^{t} \delta \mathbf{w}, \ldots,{ }^{t} \delta \mathbf{w}\right),
$$

where the first parts are the $I$-components and the remaining $l$ entries are the Jordan quiver components. In particular $\pi_{0, \zeta}^{-1}(x)$ is isomorphic to the product of $\pi_{0, \zeta}^{-1}(0)\left(\subset \mathfrak{M}_{\zeta}\left(\mathbf{w}-\mathbf{C v}^{\prime}\right)\right)$ and $\pi_{0, \zeta}^{-1}(0)\left(\subset M_{\zeta}^{\text {norm }}\left(\lambda_{i},{ }^{t} \delta \mathbf{w}\right)\right)(i=1, \ldots, l)$, where the latter are the quiver varieties associated with Jordan quivers. It is known that the latter's are irreducible and half-dimensional in $M_{\zeta}^{\text {norm }}\left(\lambda_{i},{ }^{t} \delta \mathbf{w}\right)$. (See [25, Exercise 5.15] and the references therein.) Thus $H_{\text {top }}\left(\pi_{0, \zeta}^{-1}(x)\right)$ is isomorphic to the tensor product of $H_{\text {top }}\left(\pi_{0, \zeta}^{-1}(0)\right)$ and $\mathbb{C}\left[\prod_{i} \pi_{0, \zeta}^{-1}(0)\left(\subset M_{\zeta}^{\text {norm }}\left(\lambda_{i}{ }^{t} \delta \mathbf{w}\right)\right)\right]$. The affine Lie algebra $\mathfrak{g}$ acts trivially on the second factor (or more precisely the degree operator $d$ 
acts by $-\lambda_{i}$ by Remark 5.5), so $H_{\text {top }}\left(\pi_{0, \zeta}^{-1}(x)\right)$ is isomorphic to $V^{\mathfrak{g}}\left(\mathbf{w}-\mathbf{v}^{\prime}-|\lambda| \delta\right)$. In particular, we get

$$
\left(\pi_{0, \zeta}\right)_{*}\left(\mathbb{C}_{\mathfrak{M}_{\zeta}(\mathbf{w})}[\operatorname{dim}]\right)=\bigoplus_{\mathbf{v}^{\prime}, \lambda} I C\left(\mathfrak{M}_{0}^{\mathrm{s}}\left(\mathbf{v}^{\prime}, \mathbf{w}\right) \times S_{\lambda}^{|\lambda|}\left(\mathbb{C}^{2} \backslash\{0\} / \Gamma\right)\right) \otimes V^{\mathfrak{g}}\left(\mathbf{w}-\mathbf{v}^{\prime}-|\lambda| \delta\right) .
$$

Note that the argument also shows that nontrivial local systems do not appear in the direct summand as for regular strata. Moreover, the closure of $\left.S_{\lambda}^{|\lambda|}\left(\mathbb{C}^{2} \backslash\{0\} / \Gamma\right)\right)$ has only finite quotient singularities, and hence is rationally smooth: $\left.I C\left(S_{\lambda}^{|\lambda|}\left(\mathbb{C}^{2} \backslash\{0\} / \Gamma\right)\right)\right)=\mathbb{C} \frac{}{\left.S_{\lambda}^{|\lambda|}\left(\mathbb{C}^{2} \backslash\{0\} / \Gamma\right)\right)}[\operatorname{dim}]$, where $\operatorname{dim}$ means $\left.\operatorname{dim} S_{\lambda}^{|\lambda|}\left(\mathbb{C}^{2} \backslash\{0\} / \Gamma\right)\right)$.

Hence we have

Theorem 5.10. Suppose the graph is of affine type and $I^{0} \neq I$. Then

(1) The strata of $\mathfrak{M}_{\zeta}(\mathbf{w})$ and $\mathfrak{M}_{0}(\mathbf{w})$ are given by (5.8), (5.9) respectively. The criterion of the nonemptiness of $\mathfrak{M}_{\zeta}^{\mathrm{s}}(\mathbf{v}, \mathbf{w}), \mathfrak{M}_{0}^{\mathrm{s}}(\mathbf{v}, \mathbf{w})$ is given in Proposition 2.30.

(2) We have

$$
\begin{aligned}
\left(\pi_{0, \zeta}\right)_{*}\left(I C\left(\mathfrak{M}_{\zeta^{\bullet}}^{\mathrm{s}}\left(\mathbf{v}^{0}, \mathbf{w}\right)\right)\right)= & \bigoplus_{\mathbf{v}, \lambda} I C\left(\mathfrak{M}_{0}^{\mathrm{s}}\left(\mathbf{v}^{\prime}, \mathbf{w}\right)\right) \otimes \mathbb{C} \frac{\left.\mathbb{S}_{\lambda}^{|\lambda|}\left(\mathbb{C}^{2} \backslash\{0\} / \Gamma\right)\right)}{\operatorname{dim}]} \\
& \otimes_{\mathbb{C}} \operatorname{Hom}_{\left(\mathfrak{g}_{I^{0}}+\mathfrak{h}\right)}\left(V^{\left(\mathfrak{g}_{I^{0}}+\mathfrak{h}\right)}\left(\mathbf{w}-\mathbf{v}^{0}\right), V^{\mathfrak{g}}\left(\mathbf{w}-\mathbf{v}^{\prime}-|\lambda| \delta\right)\right) .
\end{aligned}
$$

Remarks 5.11. (1) Suppose $I^{0}=\varnothing$ and hence $\zeta=\zeta^{\bullet}, \mathfrak{M}_{\zeta}^{\mathrm{s}}\left(\mathbf{v}^{0}, \mathbf{w}\right)=\mathfrak{M}_{\zeta}\left(\mathbf{v}^{0}, \mathbf{w}\right)$. Then the above implies that the restriction of $H_{*}\left(\pi_{0, \zeta}^{-1}(0)\right)$ to a $\mathfrak{g}$-module decomposes as

$$
\bigoplus_{\mathbf{v}, \lambda} H^{*}\left(i_{0}^{!} I C\left(\mathfrak{M}_{0}^{\mathrm{s}}(\mathbf{v}, \mathbf{w})\right)\right) \otimes V^{\mathfrak{g}}(\mathbf{w}-\mathbf{v}-|\lambda| \delta),
$$

where $i_{0}:\{0\} \rightarrow \mathfrak{M}_{0}(\mathbf{w})$ is the inclusion. This is nothing but [28, Theorem 5.2].

(2) It is known that $H_{*}\left(\pi_{0, \zeta}^{-1}(0)\right)$ is the tensor product of $\mathfrak{g}$-modules corresponding to ones with $\mathbf{w}=\Lambda_{\mu_{p}}$ for various $\mu_{p} \in[0, r-1]$. (See the proof of $[26,14.1 .2]$.) If we further assume $\mathfrak{g}$ is of affine type $A_{r-1}^{(1)}=\mathfrak{s l}(r)_{\text {aff }}$, then those $\mathfrak{g}$-modules can be computed by various means. For example, we use Proposition 2.30 to find $\mathfrak{M}_{0}^{\mathrm{s}}\left(\mathbf{v}, \Lambda_{\mu_{p}}\right)=\varnothing$ unless $\mathbf{v}=0$. Therefore $H_{*}\left(\pi_{0, \zeta}^{-1}(0)\right)$ is isomorphic to $\bigoplus_{\lambda} V^{\mathfrak{g}}\left(\Lambda_{\mu_{p}}-|\lambda| \delta\right)$. The direct sum of 1-dimensional spaces for each partition $\lambda$ is isomorphic to the Fock space of the Heisenberg algebra. Thus this is isomorphic to the restriction of the highest weight representation $V^{\mathfrak{g l} l}(r)_{\text {aff }}\left(\Lambda_{i}\right)$ of $\mathfrak{g l}(r)_{\text {aff }}$ to $\mathfrak{s l}(r)_{\text {aff }}$. (See Section A.) Combined with (A.6) we find that $H^{*}\left(i_{0}^{!} I C\left(\mathfrak{M}_{0}^{\mathrm{s}}(\mathbf{v}, \mathbf{w})\right)\right)$ is isomorphic to $V^{\mathfrak{s l}(l)_{\text {aff }}}(\bar{\lambda})_{\bar{\mu}}$. This observation was used in [4] to confirm a (weak form) of the conjecture proposed there in type $A$.

\subsection{Restriction to the affine Lie algebra of a Levi factor}

Let us consider the case in Section 3.2. The analysis is almost the same as in the previous subsection.

The stratification of $\mathfrak{M}_{0}(\mathbf{w})$ was already given in (5.9). The fiber $\pi_{0, \zeta}^{-1}(x)$ of a point $x$ in the stratum $\mathfrak{M}_{0}^{\mathrm{s}}\left(\mathbf{v}^{\prime}, \mathbf{w}\right) \times S_{\lambda}^{|\lambda|}\left(\mathbb{C}^{2} \backslash\{0\} / \Gamma\right)$ (see (5.9)) is isomorphic to the integrable highest weight representation $V^{\mathfrak{g}}\left(\mathbf{w}-\mathbf{v}^{\prime}-|\lambda| \delta\right)$ of the affine Lie algebra $\mathfrak{g}$. Strictly speaking, this result does not follows directly from [24] as the generic stability parameter $\zeta$ used there is different from the one used here. Rather it is the one in Section 2.8. Therefore $H_{\text {top }}\left(\pi_{0, \zeta}^{-1}(x)\right)$, a priori, only has an $\mathfrak{h}$-module structure. Let us denote the generic parameter $\zeta$ in the previous subsection by $\zeta^{+}$. 
Lemma 5.12. Let us fix $\mathbf{v}$ and consider $\pi_{0, \zeta}^{-1}(x) \subset \mathfrak{M}_{\zeta}(\mathbf{v}, \mathbf{w})$ and $\pi_{0, \zeta^{+}}^{-1}(x) \subset \mathfrak{M}_{\zeta^{+}}(\mathbf{v}, \mathbf{w})$. There is a graded vector space isomorphism $H_{*}\left(\pi_{0, \zeta}^{-1}(x)\right) \cong H_{*}\left(\pi_{0, \zeta^{+}}^{-1}(x)\right.$ ) (which is canonical in the way explained during the proof $)$. In particular, $H_{\mathrm{top}}\left(\pi_{0, \zeta}^{-1}(x)\right)$ is isomorphic to $V^{\mathfrak{g}}\left(\mathbf{w}-\mathbf{v}^{\prime}-|\lambda| \delta\right)$ as an $\mathfrak{h}$-module.

By (5.2) $H_{\text {top }}\left(Z_{0, \zeta}(\mathbf{w})\right)$ has an induced homomorphism from $\mathbf{U}(\mathfrak{g})$.

Proof. We know that $\pi_{0, \zeta}^{-1}(x) \subset \mathfrak{M}_{\zeta}(\mathbf{v}, \mathbf{w})$ is isomorphic to the product of $\pi_{0, \zeta}^{-1}(0) \subset \mathfrak{M}_{\zeta}\left(\mathbf{v}-\mathbf{v}^{\prime}\right.$, $\left.\mathbf{w}-\mathbf{c v}^{\prime}\right)$ and $\pi_{0, \zeta}^{-1}(0) \subset M_{\zeta}^{\text {norm }}\left(\lambda_{i},{ }^{t} \delta \mathbf{w}\right)(i=1, \ldots, l)$ as in the previous subsection. The same holds for $\zeta^{+}$. The latter factors are the same for our $\zeta$ and $\zeta^{+}$, so we need to worry about the first factor. We know that the inclusion $\pi_{0, \zeta}^{-1}(0) \subset \mathfrak{M}_{\zeta}\left(\mathbf{v}-\mathbf{v}^{\prime}, \mathbf{w}-\mathbf{C v}^{\prime}\right)$ and one for $\zeta^{+}$are homotopy equivalences $[22,5.5]$, and the $C^{\infty}{ }_{\text {-structure of }} \mathfrak{M}_{\zeta}\left(\mathbf{v}-\mathbf{v}^{\prime}, \mathbf{w}-\mathbf{C v}^{\prime}\right)$ is independent of the choice of generic parameter $\zeta$. In particular, we get the first assertion. Since this is the weight space, we have the second assertion.

There is another way to construct an isomorphism $H_{*}\left(\pi_{0, \zeta}^{-1}(x)\right) \cong H_{*}\left(\pi_{0, \zeta^{+}}^{-1}(x)\right)$. Let us sketch the construction. We introduce the complex parameter $\zeta \mathbb{C} \in \mathbb{C}^{I}$ and consider the quotients of $\mu^{-1}\left(\mathbb{C}^{I}\right)$ by $G$ associated with the stability parameters $\zeta, \zeta^{+}$and 0 . Let us denote the corresponding variety by $\mathfrak{N}_{\zeta}(\mathbf{v}, \mathbf{w})$, etc. The morphisms $\pi_{0, \zeta}: \mathfrak{N}_{\zeta}(\mathbf{v}, \mathbf{w}) \rightarrow \mathfrak{N}_{0}(\mathbf{v}, \mathbf{w})$, $\pi_{0, \zeta^{+}}: \mathfrak{N}_{\zeta^{+}}(\mathbf{v}, \mathbf{w}) \rightarrow \mathfrak{N}_{0}(\mathbf{v}, \mathbf{w})$ become small, and the pushforward of the constant sheaves $\mathbb{C}_{\mathfrak{N}_{\zeta}(\mathbf{v}, \mathbf{w})}[\operatorname{dim}], \mathbb{C}_{\mathfrak{N}_{\zeta^{+}}(\mathbf{v}, \mathbf{w})}[\operatorname{dim}]$ are both the $I C$ sheaf of the closure of $\mathfrak{N}_{0}^{\mathrm{s}}(\mathbf{v}, \mathbf{w})$. Therefore both $H_{*}\left(\pi_{0, \zeta}^{-1}(x)\right)$ and $H_{*}\left(\pi_{0, \zeta^{+}}^{-1}(x)\right)$ are the stalk of $I C\left(\mathfrak{N}_{0}^{\mathrm{s}}(\mathbf{v}, \mathbf{w})\right)$ at $x$, hence are isomorphic. Moreover as $H_{\text {top }}\left(Z_{0, \zeta}(\mathbf{w})\right)$ is isomorphic to $\operatorname{End}_{D^{b}\left(\mathfrak{M}_{0}(\mathbf{w})\right)}\left(\left(\pi_{0, \zeta}\right)_{*}\left(\mathbb{C}_{\mathfrak{M}_{\zeta}(\mathbf{w})}[\operatorname{dim}]\right)([7,8.9 .6])\right.$, this construction also gives a natural isomorphism $H_{\text {top }}\left(Z_{0, \zeta}(\mathbf{w})\right) \cong H_{\text {top }}\left(Z_{0, \zeta^{+}}(\mathbf{w})\right)$ respecting the convolution product. This kind of construction is well-known in the geometric construction of the Springer correspondence (see [17]). And $\mathfrak{N}_{0}(\mathbf{v}, \mathbf{w})$ is an analog of the Beilinson-Drinfeld Grassmannian in the double affine Grassmannian.

Remark 5.13. It is probably possible to define a homomorphism $\mathbf{U}(\mathfrak{g}) \rightarrow H_{\text {top }}\left(Z_{0, \zeta}(\mathbf{w})\right)$ also for this $\zeta$ more directly. When $\mathbf{w}$ is of level 1 , this can be done by combining the Heisenberg algebra representation on the cohomology group of Hilbert schemes of points [25] and the FrenkelKac construction [12, § 14] (see [10]). Furthermore, it defines the same representation on $H_{\text {mid }}\left(\mathfrak{M}_{\zeta}(\mathbf{w})\right) \cong H_{\text {mid }}\left(\mathfrak{M}_{\zeta^{+}}(\mathbf{w})\right)$ as one in [24] if the graph is of affine type $A$ [21].

Since $V^{\mathfrak{g}}\left(\mathbf{w}-\mathbf{v}^{\prime}-|\lambda| \delta\right)$ is not irreducible as an $\mathfrak{h}$-module, the previous argument showing that $V_{(\widehat{G}), \phi}=0$ unless $\phi$ is the trivial local system does not work. But we can deduce it from the corresponding vanishing for $\zeta^{+}$in the previous subsection. This can be done (at least) in two ways. One is to use the reflection functor [29] to construct an isomorphism $\mathfrak{M}_{\zeta}(\mathbf{v}, \mathbf{w}) \rightarrow$ $\mathfrak{M}_{\zeta^{+}}\left(\mathbf{v}^{\prime}, \mathbf{w}\right)$ (for an appropriate $\mathbf{v}^{\prime}$ ). It is compatible with $\pi_{0, \zeta}, \pi_{0, \zeta^{+}}$, so the assertion follows. The second one is to use $\mathfrak{N}_{\zeta}$ as above. Then both $\left(\pi_{0, \zeta}\right)_{*}\left(\mathbb{C}_{\mathfrak{M}_{\zeta}(\mathbf{v}, \mathbf{w})}[\operatorname{dim}]\right),\left(\pi_{0, \zeta^{+}}\right)_{*}\left(\mathbb{C}_{\mathfrak{M}_{\zeta+}(\mathbf{v}, \mathbf{w})}\right)$ are obtained from the above IC sheaf $\operatorname{IC}\left(\mathfrak{N}_{0}^{\mathrm{s}}(\mathbf{v}, \mathbf{w})\right)$ by the restriction. Therefore they are isomorphic.

Next consider $\pi_{\zeta}, \zeta$. The stratification of $\mathfrak{M}_{\zeta} \bullet(\mathbf{w})$ induced from (3.5) is

$$
\mathfrak{M}_{\zeta^{\bullet}}(\mathbf{w})=\bigsqcup \mathfrak{M}_{\zeta^{\bullet}}^{\mathrm{s}}\left(\mathbf{v}^{0}, \mathbf{w}\right) \times S_{\mu}^{|\mu|}\left[X_{\zeta^{\circ}} \backslash \bigcup_{i \in I_{0}^{0}} C_{i}\right]
$$

where $\mu$ is a partition.

Take a point $y$ from the stratum of the form in (5.14). The fiber $\pi_{\zeta}^{-1}, \zeta(y)$ is isomorphic to the product of $\pi_{0, \zeta}^{-1}(0) \subset \mathfrak{M}_{\zeta}\left(\widehat{\mathbf{w}}_{c}\right)(c \in \mathcal{C})$ and $\pi_{0, \zeta}^{-1}(0) \subset M_{\zeta}^{\text {norm }}\left(\mu_{i},{ }^{t} \delta \mathbf{w}\right)(i=1, \ldots, l)$. Here $\widehat{\mathbf{w}}_{c}$ is the entries corresponding to $c$ in (3.6), and $\mathfrak{M}_{\zeta}\left(\widehat{\mathbf{w}}_{c}\right)$ is the quiver variety for the affine graph 
corresponding to $c$. Let $\left(\mathfrak{g}_{I_{0}^{0}}\right)_{\text {aff }}$ be the affine Lie algebra of the Levi factor $\mathfrak{g}_{I_{0}^{0}}$ of the finite dimensional Lie algebra $\mathfrak{g}_{I^{0}}$. Then $H_{\text {top }}\left(\pi_{\zeta^{\bullet}, \zeta}^{-1}(y)\right)$ is the integrable highest weight representation $V^{\left(\mathfrak{g}_{I_{0}^{0}}\right)_{\mathrm{aff}}+\mathfrak{h}}\left(\mathbf{w}-\mathbf{v}^{0}-|\mu| \delta\right)$. More precisely it is the direct product of the integrable highest weight representation for the affine Lie algebra corresponding to $c$ with the highest weight $\widehat{\mathbf{w}}_{c}$ for $c \in \mathcal{C}$. Note that each factor has level ${ }^{t} \delta \mathbf{w}$, independent of $c$, thus the affine Lie algebra $\left(\mathfrak{g}_{I_{0}^{0}}\right)_{\text {aff }}$ contains only the one dimensional central extension even if $\mathfrak{g}_{I_{0}^{0}}$ has several simple factors.

As in the case of $\pi_{0, \zeta}$ we need an explanation of the $\left(\left(\mathfrak{g}_{I_{0}^{0}}\right)_{\text {aff }}+\mathfrak{h}\right)$-module structure on $H_{\text {top }}\left(\pi_{\zeta^{\bullet}, \zeta}^{-1}(y)\right)$. It can be done in two ways as above. We still need to compare it with the $\mathfrak{g}$-module structure considered for $\pi_{0, \zeta}$. It is clear that the $\mathfrak{h}$-module structure is compatible in either ways. But the author does not know how to compare the $\mathfrak{g}$-module and $\left(\mathfrak{g}_{I_{0}^{0}}\right)_{\text {aff }}$-module structure. Therefore the equality (5.7) holds only as $\mathfrak{h}$-modules. Practically it is enough for our purpose as integrable highest weight representations are determined by their characters. Thus we get

Theorem 5.15. (1) The strata of $\mathfrak{M}_{\zeta} \bullet(\mathbf{w})$ and $\mathfrak{M}_{0}(\mathbf{w})$ are given by (5.14), (5.9) respectively. The criterion of the nonemptiness of $\mathfrak{M}_{\zeta}^{\mathrm{s}} \cdot(\mathbf{v}, \mathbf{w}), \mathfrak{M}_{0}^{\mathrm{s}}(\mathbf{v}, \mathbf{w})$ is given in Proposition 3.8.

(2) If we define $V_{\mathbf{v}^{\prime}, \lambda}^{\mathbf{v}^{0}, \mu}$ by

$$
\begin{aligned}
& \left(\pi_{0, \zeta}\right)_{*}\left(I C\left(\mathfrak{M}_{\zeta^{\bullet}}^{\mathrm{s}}\left(\mathbf{v}^{0}, \mathbf{w}\right)\right) \otimes \mathbb{C} \frac{}{S_{\mu}^{|\mu|}\left[X_{\zeta^{\circ}} \backslash \bigcup_{i \in I_{0}^{0}} C_{i}\right]}[\operatorname{dim}]\right) \\
& =\bigoplus_{\mathbf{v}^{\prime}, \lambda} I C\left(\mathfrak{M}_{0}^{\mathrm{s}}\left(\mathbf{v}^{\prime}, \mathbf{w}\right)\right) \otimes \mathbb{C} \frac{}{\left.S_{\lambda}^{|\lambda|}\left(\mathbb{C}^{2} \backslash\{0\} / \Gamma\right)\right)}[\operatorname{dim}] \otimes V_{\mathbf{v}^{\prime}, \lambda}^{\mathbf{v}^{0}, \mu},
\end{aligned}
$$

we have

$$
\bigoplus_{\mathbf{v}^{0}, \mu} V_{\mathbf{v}^{\prime}, \lambda}^{\mathbf{v}^{0}, \mu} \otimes V^{\left(\left(\mathfrak{g}_{I_{0}^{0}}\right)_{\text {aff }}+\mathfrak{h}\right)}\left(\mathbf{w}-\mathbf{v}^{0}-|\mu| \delta\right) \cong V^{\mathfrak{g}}\left(\mathbf{w}-\mathbf{v}^{\prime}-|\lambda| \delta\right) .
$$

Therefore

$$
\operatorname{Hom}_{\left(\mathfrak{g}_{I_{0}^{0}}\right)_{\text {aff }}+\mathfrak{h}}\left(V^{\left(\left(\mathfrak{g}_{I_{0}^{0}}\right)_{\text {aff }}+\mathfrak{h}\right)}\left(\mathbf{w}-\mathbf{v}^{0}\right), V^{\mathfrak{g}}\left(\mathbf{w}-\mathbf{v}^{\prime}-|\lambda| \delta\right)\right)=\bigoplus_{\mu} V_{\mathbf{v}^{\prime}, \lambda}^{\mathbf{v}^{0}-|\mu| \delta, \mu} .
$$

Remarks 5.17. (1) By the same argument as in the proof of Theorem 5.6 we have $V_{\mathbf{v}^{\prime}, \varnothing}^{\mathbf{v}^{0}, \mu}=0$ unless $\mu=\varnothing$. Therefore the right hand side of (5.16) contains only the single term $V_{\mathbf{v}^{\prime}, \lambda}^{\mathbf{v}^{0}, \varnothing}$ if $\lambda=\varnothing$.

(2) We can determine the individual $V_{\mathbf{v}^{\prime}, \lambda}^{\mathbf{v}^{0}, \mu}$ from the branching coefficients. First we show that that it is enough to consider the case $\mu=\varnothing$. For a general $\mu$, we use the diagram (2.29) and argue as in the proof of Theorem 5.6. We need to compute

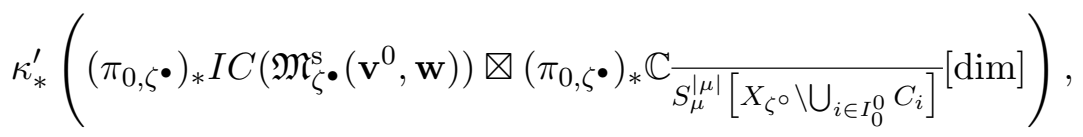

where the first $\pi_{0, \zeta}$ is $\pi_{0, \zeta}: \mathfrak{M}_{\zeta}\left(\mathbf{v}^{0}, \mathbf{w}\right) \rightarrow \mathfrak{M}_{0}\left(\mathbf{v}^{0}, \mathbf{w}\right)$ and the second one is $\pi_{0, \zeta}: S^{|\mu|} X_{\zeta} \bullet \rightarrow$ $S^{|\mu|} \mathbb{C}^{2} / \Gamma$. The first pushforward is already known, as it is the case $\mu=\varnothing$. The second pushforward is easily computed as $\pi_{0, \zeta}$ is semismall, both $S^{|\mu|} X_{\zeta}, S^{|\mu|} \mathbb{C}^{2} / \Gamma$ are rationally smooth, and fibers can be described. Finally we can compute

$$
\kappa_{*}^{\prime}\left(I C\left(\mathfrak{M}_{\zeta}^{\mathrm{s}} \cdot\left(\mathbf{v}^{0}, \mathbf{w}\right)\right) \otimes \mathbb{C} \frac{}{S_{\lambda}^{|\lambda|}\left(\mathbb{C}^{2} \backslash\{0\} / \Gamma\right)} \otimes \mathbb{C} \frac{}{S_{\lambda^{\prime}}^{\left|\lambda^{\prime}\right|}\left(\mathbb{C}^{2} \backslash\{0\} / \Gamma\right)}\right)
$$


by calculating the degree of the map $\kappa^{\prime}: S_{\lambda}^{|\lambda|}\left(\mathbb{C}^{2} \backslash\{0\} / \Gamma\right) \times S_{\lambda^{\prime}}^{\left|\lambda^{\prime}\right|}\left(\mathbb{C}^{2} \backslash\{0\} / \Gamma\right) \rightarrow S_{\lambda \cup \lambda^{\prime}}^{|\lambda|+\left|\lambda^{\prime}\right|}\left(\mathbb{C}^{2} \backslash\right.$ $\{0\} / \Gamma)$.

When we consider a string ' $\mathbf{v}^{0}+\mathbb{Z} \delta$ ', there is a minimal element $\mathbf{v}_{\text {min }}^{0}$ such that $\mathfrak{M}_{\zeta}^{\mathrm{s}} \cdot\left(\mathbf{v}_{\text {min }}^{0}-\right.$ $m \delta, \mathbf{w})=\varnothing$ for $m>0$. Then the right hand side only has a single summand $V_{\mathbf{v}^{\prime}, \lambda}^{\mathbf{v}_{\text {in }}^{0}, \varnothing}$ if $\mathbf{v}^{0}=\mathbf{v}_{\min }^{0}$. Thus it is determined by the branching coefficient. For general $\mathbf{v}^{0}=\mathbf{v}_{\min }^{0}+m \delta$, we can determine $V_{\mathbf{v}^{\prime}, \lambda}^{\mathbf{v}^{0}, \varnothing}$ by induction on $m$, as summands appearing in the right hand side of (5.16) are smaller.

(3) Suppose that $\mathfrak{g}$ is of affine type $A_{r-1}^{(1)}=\mathfrak{s l}(r)_{\text {aff }}$ and $I_{0}^{0}=I_{0} \backslash\left\{r_{1}\right\}$ for some $1 \leq r_{1}<r$. Then we have $\mathfrak{g}_{I_{0}^{0}} \cong \mathfrak{s l}\left(r_{1}\right) \oplus \mathfrak{s l}\left(r_{2}\right)$ where $r_{2}=r-r_{1}$. By (A.9) the factor $\operatorname{Hom}_{\left(\left(\mathfrak{g}_{I_{0}}\right)_{\text {aff }}+\mathfrak{h}\right)}\left(V^{\left(\left(\mathfrak{g}_{I_{0}^{0}}\right)_{\text {aff }}+\mathfrak{h}\right)}\right.$

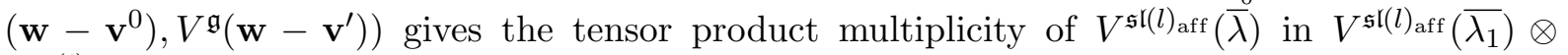
$V^{\mathfrak{s l} l(l)}$ aff $\left(\overline{\lambda_{2}}\right)$ for appropriate $\bar{\lambda}, \overline{\lambda_{1}}, \overline{\lambda_{2}}$. This confirms the conjecture [5] that the convolution diagram for the double affine Grassmannian realizing the tensor product is defined by the Uhlenbeck partial compactification of the framed moduli space of instantons on the orbifold $X_{\zeta} \bullet$ However it remains to be clarified how we should interpret summands $\lambda, \mu \neq \varnothing$ to make a 'categorical' statement as in the usual geometric Satake correspondence.

(4) In view of the level-rank duality it is natural to expect that $H_{*}\left(\pi_{0, \zeta}^{-1}(0)\right)$ has a structure of the Heisenberg algebra module commuting with the $\mathfrak{g}$-action. Since $\mathfrak{M}_{\zeta}(\mathbf{w})$ is the framed moduli space of torsion free sheaves on $X_{\zeta^{\circ}}$, such a structure was constructed by the author [25, Chapter 8] (see [2] for a higher rank generalization). When $\mathbf{w}$ is of level 1, one can check that the Heisenberg algebra action commutes with the $\mathfrak{g}$-action mentioned in Remark 5.13, but it is still open in higher level.

\section{MV cycles for the double affine Grassmannian of type $A$}

The Mirković-Vilonen cycles (MV cycles in short) are certain subvarieties in the affine Grassmannian and are natural geometric basis elements of a weight space of an irreducible finite dimensional representation of a finite dimensional simple Lie algebra [20]. Their conjectural double affine Grassmannian analogs are proposed by Braverman and Finkelberg [5].

Via the level-rank duality (see (A.6)) their conjectural basis for $V^{\mathfrak{s} r(l)_{\text {aff }}}(\bar{\lambda})_{\bar{\mu}}$ also should give a basis of the tensor product multiplicity space for $\mathfrak{g l}(r)_{\text {aff }}$. Recall that the author introduced a Lagrangian subvariety $\widetilde{\mathfrak{Z}}(\mathbf{w})$ in $\bigsqcup_{\mathbf{v}} \mathfrak{M}_{\zeta}(\mathbf{v}, \mathbf{w})$ such that the set of its irreducible components have a crystal structure isomorphic to the tensor product for $\mathfrak{s l}(r)_{\text {aff }}$ [27] (see also [19]). A simple modification gives the tensor product for $\mathfrak{g l}(r)_{\text {aff }}$. Then we show that those irreducible components of (the modified version of) $\widetilde{\mathfrak{Z}}(\mathbf{w})$ intersecting with the open subvariety $\bigcup \pi^{-1}\left(\mathfrak{M}_{0}^{\mathrm{s}}(\mathbf{v}, \mathbf{w})\right)$ are exactly highest weight vectors. In particular, the number of such irreducible components is equal to the weight multiplicity. Note also that those irreducible components are identified with those of a Lagrangian subvariety in $\mathfrak{M}_{0}^{\mathrm{s}}(\mathbf{v}, \mathbf{w})$. The definition makes sense for the Uhlenbeck compactification of the framed moduli of $G$-bundles for any $G$.

Remark 6.1. Together with the theory of the crystal base, we have the actual highest weight vectors in the tensor product representation (instead of the tensor product crystal) parametrized naturally by those irreducible components. But the fundamental classes of those irreducible components are not necessarily highest weight vectors, when we realize the tensor product representation as the homology group of $\widetilde{\mathfrak{Z}}(\mathbf{w})$ [27]. Note also that $\widetilde{\mathfrak{Z}}(\mathbf{w})$ makes sense only for $G=S L(l)$ (or $G L(l))$. We do not know a satisfactory natural way to remedy this flaw.

Let $(I, E)$ be the graph of the affine type $A_{r-1}$. We number the vertices in the cyclic order as usual starting from 0 to $r-1$. We choose the cyclic orientation $\Omega$, i.e., $0 \rightarrow 1,1 \rightarrow 2, \ldots$, $r-2 \rightarrow r-1, r-1 \rightarrow 0$. The corresponding Lie algebra $\mathfrak{g}$ is $\mathfrak{s l}(r)_{\text {aff }}$. 
We choose the stability parameter $\zeta$ as in Example 2.7(1). For $I$-graded vector spaces $V$, $W$ with $\mathbf{v}=\operatorname{dim} V, \mathbf{w}=\operatorname{dim} W$ we consider quiver varieties $\mathfrak{M}_{\zeta}(\mathbf{v}, \mathbf{w}), \mathfrak{M}_{0}(\mathbf{v}, \mathbf{w})$. We further choose a decomposition $W=W^{1} \oplus W^{2} \oplus \cdots \oplus W^{l}$ into 1-dimensional subspaces. (At the end the definition of MV cycles depends only on the flag $0 \subset W^{1} \subset W^{1} \oplus W^{2} \subset \cdots \subset W$.) In particular, $W^{p}$ is concentrated at a degree $\mu_{p} \in I$, i.e., $\operatorname{dim} W^{p}=\mathbf{e}_{\mu_{p}}$. We define a 1-parameter subgroup $\rho_{0}: \mathbb{C}^{*} \rightarrow G_{W}$ by

$$
\rho_{0}(t)=t^{m_{1}} \operatorname{id}_{W^{1}} \oplus t^{m_{2}} \operatorname{id}_{W^{2}} \oplus \cdots \oplus t^{m_{l}} \mathrm{id}_{W^{l}}
$$

with $m_{1} \ll m_{2} \ll \cdots \ll m_{l}$. We define a $\mathbb{C}^{*}$-action on $\mathbf{M}(V, W)$ and the induced actions on $\mathfrak{M}_{\zeta}(\mathbf{v}, \mathbf{w}), \mathfrak{M}_{0}(\mathbf{v}, \mathbf{w})$ by

$$
B_{h} \mapsto\left\{\begin{array}{ll}
B_{h} & \text { if } h \in \Omega, \\
t B_{h} & \text { if } h \in \bar{\Omega},
\end{array} \quad a \mapsto a \rho_{0}(t)^{-1}, \quad b \mapsto t \rho_{0}(t) b .\right.
$$

Let

$$
\mathfrak{Z}_{\zeta}(\mathbf{v}, \mathbf{w}) \stackrel{\text { def }}{=}\left\{[B, a, b] \in \mathfrak{M}_{\zeta}(\mathbf{v}, \mathbf{w}) \mid \lim _{t \rightarrow \infty} t \cdot[B, a, b] \text { exists }\right\} .
$$

By $[27, \S 8] \mathfrak{Z}_{\zeta}(\mathbf{v}, \mathbf{w})$ is a Lagrangian subvariety in $\mathfrak{M}_{\zeta}(\mathbf{v}, \mathbf{w})$. Let $\operatorname{Irr} \mathfrak{Z}_{\zeta}(\mathbf{v}, \mathbf{w})$ denote the set of irreducible components of $\mathfrak{Z}_{\zeta}(\mathbf{v}, \mathbf{w})$. By $[27, \S 8]$, its disjoint union $\bigsqcup_{\mathbf{v}} \operatorname{Irr} \mathfrak{Z}_{\zeta}(\mathbf{v}, \mathbf{w})$ has

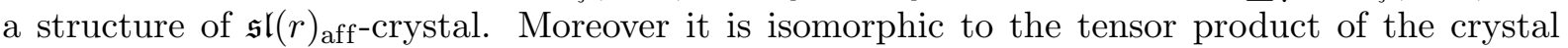
$\bigsqcup_{\mathbf{v}^{p}} \operatorname{Irr} \mathfrak{Z}_{\zeta}\left(\mathbf{v}^{p}, \Lambda_{\mu_{p}}\right)$ by the same proof as $[27,4.6]$. Here $\mathfrak{Z}_{\zeta}\left(\mathbf{v}^{p}, \Lambda_{\mu_{p}}\right)$ is defined in the same way as above applied to $\mathbf{w}=\Lambda_{\mu_{p}}$. $(\rho(t)$ does not matter in this case.)

Thanks to the following lemma, we have isomorphisms:

$$
\bigsqcup_{\mathbf{v}} \operatorname{Irr} \mathfrak{Z}_{\zeta}(\mathbf{v}, \mathbf{w}) \cong \bigotimes_{p=1}^{l}\left(\bigoplus_{\lambda^{p}} \mathscr{B}^{\mathfrak{s l}(r))_{\mathrm{aff}}}\left(\Lambda_{\mu_{p}}\right) \otimes T_{-\left|\lambda^{p}\right| \delta}\right),
$$

where $\lambda^{p}$ runs over partitions. Here $\mathscr{B}^{\mathfrak{s}(r)_{\text {aff }}}\left(\Lambda_{\mu_{p}}\right)$ denotes the crystal of the integrable highest weight representation $V^{\mathfrak{s l}(r)}$ aff $\left(\Lambda_{\mu_{p}}\right)$, and $T_{-\left|\lambda^{p}\right| \delta}$ is the crystal consisting of a single element with weight $-\left|\lambda^{p}\right| \delta$.

Lemma 6.3. $\bigsqcup_{\mathbf{v}^{p}} \operatorname{Irr} \mathfrak{Z}_{\zeta}\left(\mathbf{v}^{p}, \Lambda_{\mu_{p}}\right)$ is isomorphic to $\bigoplus_{\lambda^{p}} \mathscr{B}^{\mathfrak{s l}(r)_{\text {aff }}}\left(\Lambda_{\mu_{p}}\right) \otimes T_{-\left|\lambda^{p}\right| \delta}$.

We now introduce the analog of MV cycles:

$$
\mathfrak{Z}_{0}^{\mathrm{s}}(\mathbf{v}, \mathbf{w}) \stackrel{\text { def }}{=}\left\{[B, a, b] \in \mathfrak{M}_{0}^{\mathrm{s}}(\mathbf{v}, \mathbf{w}) \mid \lim _{t \rightarrow \infty} t \cdot[B, a, b] \text { exists }\right\} .
$$

Since $\pi=\pi_{0, \zeta}$ is projective, we have $\mathfrak{Z}_{0}^{\mathrm{s}}(\mathbf{v}, \mathbf{w})=\pi\left(\mathfrak{Z}_{\zeta}(\mathbf{v}, \mathbf{w})\right) \cap \mathfrak{M}_{0}^{\mathrm{s}}(\mathbf{v}, \mathbf{w})$. Since $\pi$ is an isomorphism on $\pi^{-1}\left(\mathfrak{M}_{0}^{\mathrm{s}}(\mathbf{v}, \mathbf{w})\right.$ ) (see Section 2.5), this can be further identified with $\mathfrak{Z}_{\zeta}(\mathbf{v}, \mathbf{w}) \cap$ $\pi^{-1}\left(\mathfrak{M}_{0}^{\mathrm{s}}(\mathbf{v}, \mathbf{w})\right)$. Since $\pi^{-1}\left(\mathfrak{M}_{0}^{\mathrm{s}}(\mathbf{v}, \mathbf{w})\right)$ is an open subset, $\mathfrak{Z}_{0}^{\mathrm{S}}(\mathbf{v}, \mathbf{w})$ is of pure dimension with $\operatorname{dim}=\operatorname{dim} \mathfrak{M}_{\zeta}(\mathbf{v}, \mathbf{w}) / 2$. Its irreducible components are naturally identified with irreducible components of $\mathfrak{Z}_{\zeta}(\mathbf{v}, \mathbf{w})$ intersecting with $\pi^{-1}\left(\mathfrak{M}_{0}^{\mathrm{s}}(\mathbf{v}, \mathbf{w})\right)$.

Theorem 6.4. (1) $\mathfrak{Z}_{0}^{\mathrm{s}}(\mathbf{v}, \mathbf{w}) \cong \mathfrak{Z}_{\zeta}(\mathbf{v}, \mathbf{w}) \cap \pi^{-1}\left(\mathfrak{M}_{0}^{\mathrm{s}}(\mathbf{v}, \mathbf{w})\right)$ is of pure dimension with $\operatorname{dim}=$ $\operatorname{dim} \mathfrak{M}_{\zeta}(\mathbf{v}, \mathbf{w}) / 2$.

(2) Let $Y_{0}$ be an an irreducible component of $\mathfrak{Z}_{0}^{\mathrm{s}}(\mathbf{v}, \mathbf{w})$ and $\mathscr{B}\left(Y_{0}\right)$ be the connected component containing the closure of $\pi_{\zeta, 0}^{-1}\left(Y_{0}\right)$. Then we have

$$
\bigsqcup_{\mathbf{v}} \operatorname{Irr} \mathfrak{Z}_{\zeta}(\mathbf{v}, \mathbf{w}) \cong \bigsqcup_{\substack{Y_{0} \in \bigsqcup_{\mathbf{v}} \operatorname{Irr} \mathfrak{Z}_{0}^{\mathbf{s}}(\mathbf{v}, \mathbf{w}) \\ \lambda: \operatorname{partition}}} \mathscr{B}\left(Y_{0}\right) \otimes T_{-|\lambda| \delta}
$$

Furthermore $\mathscr{B}\left(Y_{0}\right)$ is isomorphic to the crystal of the integrable highest weight module $V^{\text {sll }(r)}$ aff (wt $\left.Y_{0}\right)$ so that $Y_{0}$ is the highest weight vector. 
Since the crystal of $V^{\mathfrak{g l}(r)_{\text {aff }}}\left(\right.$ wt $\left.Y_{0}\right)$ is isomorphic to $\sqcup_{\lambda} \mathscr{B}\left(Y_{0}\right) \otimes T_{-|\lambda| \delta}$, we conclude that

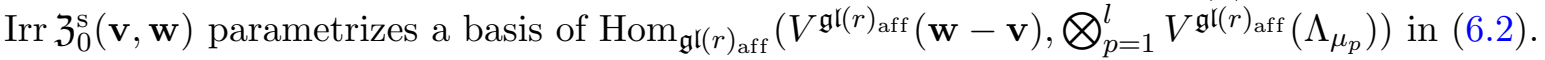

Proof of Lemma 6.3. We consider $\mathfrak{M}_{0}\left(\mathbf{v}, \Lambda_{\mu_{p}}\right)$. Thanks to Proposition 2.30(1), the stratification (3.5) is very simple in this case:

$$
\mathfrak{M}_{0}\left(\mathbf{v}, \Lambda_{\mu_{p}}\right) \cong \bigsqcup_{\lambda, m_{i}} S_{\lambda}^{|\lambda|}\left(\mathbb{C}^{2} \backslash\{0\} / \Gamma\right) \times\left\{\bigoplus_{i} S_{i}^{\oplus m_{i}}\right\}
$$

where the summation runs over partitions $\lambda$ and nonnegative integers $m_{i}$ with $\mathbf{v}=|\lambda| \delta+\sum m_{i} \mathbf{e}_{i}$. In this description, the $\mathbb{C}^{*}$-action is the induced action from the action $t \cdot(x, y) \mapsto(x, t y)$ on $\mathbb{C}^{2}$. Therefore the points where the limit exists when $t \rightarrow \infty$ are

$$
\bigsqcup_{\lambda, m_{i}} S_{\lambda}^{|\lambda|}((x \text {-axis }) \backslash\{0\} / \Gamma) \times\left\{\bigoplus_{i} S_{i}^{\oplus m_{i}}\right\} .
$$

From this description and the definition of the crystal structure, we know that the highest weight vectors, i.e., those irreducible components $Y$ with $\varepsilon_{i}(Y)=0$ for all $i \in I$, are closures of $\pi^{-1}\left(S_{\lambda}^{|\lambda|}((x\right.$-axis $) \backslash\{0\} / \Gamma)$ in $\mathfrak{M}_{\zeta}\left(|\lambda| \delta, \Lambda_{\mu_{p}}\right)$. Note that this is irreducible as the punctual Hilbert scheme is irreducible. Moreover the component of the crystal containing this highest weight vector is isomorphic to the crystal of $\bigsqcup_{\mathbf{v}^{p}} \operatorname{Irr} \mathfrak{L}_{\zeta}\left(\mathbf{v}^{p}, \Lambda_{\mu_{p}}\right) \otimes T_{-|\lambda| \delta}$. Thus we have

$$
\bigsqcup_{\mathbf{v}^{p}} \operatorname{Irr} \mathfrak{Z}_{\zeta}\left(\mathbf{v}^{p}, \Lambda_{\mu_{p}}\right) \cong \bigoplus_{\lambda^{p}}\left(\bigsqcup_{\mathbf{v}^{p}} \operatorname{Irr} \mathfrak{L}_{\zeta}\left(\mathbf{v}^{p}, \Lambda_{\mu_{p}}\right)\right) \otimes T_{-|\lambda| \delta}
$$

where $\mathfrak{L}_{\zeta}\left(\mathbf{v}^{p}, \Lambda_{\mu_{p}}\right)=\pi^{-1}(0)$. Since $\bigsqcup_{\mathbf{v}^{p}} \operatorname{Irr} \mathfrak{L}_{\zeta}\left(\mathbf{v}^{p}, \Lambda_{\mu_{p}}\right)$ is isomorphic to the crystal of $\left.V^{\mathfrak{s l}(r)}\right)_{\text {aff }}\left(\Lambda_{\mu_{p}}\right)$ $[13,33,27]$, we have the assertion.

Proof of Theorem 6.4. (1) is already proved.

(2) Let us consider the stratification of $\mathfrak{M}_{0}(\mathbf{v}, \mathbf{w})$ in $(3.5)$ :

$$
\mathfrak{M}_{0}(\mathbf{v}, \mathbf{w})=\bigsqcup \mathfrak{M}_{0}^{\mathrm{s}}\left(\mathbf{v}^{0}, \mathbf{w}\right) \times S_{\lambda}^{|\lambda|}\left(\mathbb{C}^{2} \backslash\{0\} / \Gamma\right) \times\left\{\bigoplus_{i \in I} S_{i}^{\oplus m_{i}}\right\} .
$$

The points where the limit exists when $t \rightarrow \infty$ are

$$
\bigsqcup \mathfrak{Z}_{0}^{\mathrm{s}}\left(\mathbf{v}^{0}, \mathbf{w}\right) \times S_{\lambda}^{|\lambda|}((x \text {-axis }) \backslash\{0\} / \Gamma) \times\left\{\bigoplus_{i \in I} S_{i}^{\oplus m_{i}}\right\}
$$

as in the proof of Lemma 6.3. The highest weight vectors are of the closures of

$$
\pi^{-1}\left(Y_{0} \times S_{\lambda}^{|\lambda|}((x \text {-axis }) \backslash\{0\} / \Gamma)\right)
$$

in $\mathfrak{M}_{\zeta}\left(\mathbf{v}^{0}+|\lambda| \delta, \mathbf{w}\right)$ where $Y_{0}$ is an irreducible component of $\mathfrak{Z}_{0}^{\mathrm{s}}\left(\mathbf{v}^{0}, \mathbf{w}\right)$. From the definition of the crystal structure, the connected component containing the above vector is the tensor product $\mathscr{B}\left(Y_{0}\right) \otimes T_{-|\lambda| \delta}$. This shows the first statement.

Again from the definition of the crystal structure, $\mathscr{B}\left(Y_{0}\right)$ is isomorphic to the crystal $\bigsqcup_{\mathbf{v}}$ $\operatorname{Irr} \mathfrak{L}_{\zeta}\left(\mathbf{v}, \mathbf{w}-\mathbf{v}^{0}\right)$, which is known to be isomorphic to the crystal of $V^{\mathfrak{s l}(r)}$ aff $\left(\mathbf{w}-\mathbf{v}^{0}\right)[13,33,27]$. This shows the second statement. 


\section{A Level-rank duality}

Our formulation follows [11, 31].

We denote the central extension of the loop Lie algebra by $\widehat{\mathfrak{s l}}(r)$ while the affine Lie algebra is denoted by $\mathfrak{s l}(r)_{\text {aff }}$. The latter contains the degree operator $d$. Our notation is slightly different from one in [4] and distinguishes weights of $\mathfrak{s l}(r)_{\text {aff }}$ and $\mathfrak{g l}(r)_{\text {aff }}$.

\section{A.1 Weight multiplicities}

Let $X, Y$ be finite dimensional vector spaces of dimensions $l, r$ respectively. Let $\mathscr{L}(X \otimes Y)=$ $X \otimes Y \otimes t^{1 / 2} \mathbb{C}\left[t, t^{-1}\right]$. Let $F \equiv \bigwedge^{\infty / 2} \mathscr{L}(X \otimes Y)$ be the semi-infinite wedge space, or the fermionic Fock space. If we take a basis $\left\{x_{p}\right\}(p=1, \ldots, l)$ of $X$ and $\left\{y_{i}\right\}(i=1, \ldots, r)$ of $Y, \mathscr{L}(X \otimes Y)$ has a basis $\left\{x_{p} \otimes y_{i} \otimes t^{n}\right\}(p=1, \ldots, l, i=1, \ldots, r, n \in \mathbb{Z}+1 / 2)$. We put a total ordering on the basis elements by the lexicographic ordering, first read $n$, then $p$, finally $i$. Then $F$ has a basis

$$
v_{1} \wedge v_{2} \wedge v_{3} \wedge \cdots
$$

where $v_{k}=x_{p_{k}} \otimes y_{i_{k}} \otimes t^{n_{k}}$ is a basis element in $\mathscr{L}(X \otimes Y)$ and we require that $v_{1}>v_{2}>v_{3}>\cdots$ and $v_{k+1}$ is the next element of $v_{k}$ for $k \gg 0$. We have the fermion operators $\psi^{i p}(n)$ and their conjugate operators $\psi_{i p}(n)$ acting on $F$ satisfying the Clifford algebra relations

$$
\left\{\psi^{i p}(n), \psi^{j q}(m)\right\}=0=\left\{\psi_{i p}(n), \psi_{j q}(m)\right\}, \quad\left\{\psi^{i p}(n), \psi_{j q}(m)\right\}=\delta_{i j} \delta_{p q} \delta_{m+n, 0} .
$$

The vacuum vector $|0\rangle \in F$ is the basis vector (A.1) where $v_{k}$ runs over all $x_{p} \otimes y_{i} \otimes t^{n}$ with $n<0$.

The basis vectors above are parametrized by Maya diagrams $M$ of $(l \times r)$-components:

$$
M=\left\{\begin{array}{l|l}
m_{i p}(n) & \begin{array}{l}
m_{i p}(n)=\square \text { or } \square(1 \leq p \leq l, 1 \leq i \leq r, n \in \mathbb{Z}+1 / 2) \\
m_{i p}(n)=\square(\text { resp. } \square) \text { for } n \ll 0(\text { resp. } n \gg 0)
\end{array}
\end{array}\right\} .
$$

It can be visualized as

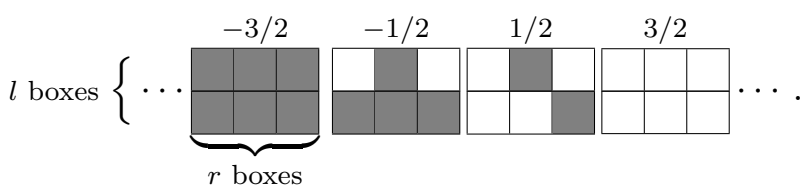

The corresponding basis element (A.1) is determined so that $v_{1}$ corresponds to the first reading from the right, $v_{2}$ corresponds to the second, etc.

We define the degree operator $d$ acting on $F$ by

$$
d(M)=\left(-\sum_{\substack{n>0 \\ m_{i, p}(n)=\square}} n+\sum_{\substack{n<0 \\ m_{i, p}(n)=\square}} n\right) M .
$$

We have $\left[d, \psi^{i p}(n)\right]=n \psi^{i p}(n),\left[d, \psi_{i p}(n)\right]=n \psi_{i p}(n)$.

We have commuting actions of $\widehat{\mathfrak{s l}}(X)_{r}, \widehat{\mathfrak{s l}}(Y)_{l}$ (the scripts indicate the levels) and the Heisenberg algebra $\widehat{a}$ given by

$$
\begin{array}{cl}
\widehat{\mathfrak{s l}}(X)_{r} \quad J_{q}^{p}(n) & (p \neq q), \quad J_{p}^{p}(n)-J_{p+1}^{p+1}(n)(p=1, \ldots, l-1), \\
& J_{p}^{q}(n) \stackrel{\text { def }}{=} \sum_{i=1}^{l} \sum_{m}: \psi^{i p}(n-m) \psi_{i q}(m):
\end{array}
$$




$$
\begin{array}{ll}
\widehat{\mathfrak{s l}}(Y)_{l} \quad J_{j}^{i}(n) & (i \neq j), \quad J_{i}^{i}(n)-J_{i+1}^{i+1}(n)(i=1, \ldots, r-1), \\
& J_{i}^{j}(n) \stackrel{\text { def }}{=} \sum_{p=1}^{r} \sum_{m}: \psi^{i p}(n-m) \psi_{j p}(m):, \\
\widehat{a} \quad & J(n) \stackrel{\text { def }}{=} \sum_{i=1}^{r} \sum_{p=1}^{l} \sum_{m}: \psi^{i p}(n-m) \psi_{i p}(m):=\sum_{i=1}^{r} J_{i}^{i}(n)=\sum_{p=1}^{l} J_{p}^{p}(n),
\end{array}
$$

where : : denotes the normal ordering, defined by $: x(m) y(n): \stackrel{\text { def }}{=} x(m) y(n)$ (resp. $y(n) x(m)$, $1 / 2(x(m) y(n)+y(n) x(m)))$ if $n>m$ (resp. $n<m, n=m)$. The degree operator $d$ gives the degree operators for $\widehat{\mathfrak{s l}}(X), \widehat{\mathfrak{s l}}(Y)$ and $\widehat{a}$.

The branching formula of $F$ is as follows [11]:

$$
F \cong \bigoplus_{\lambda \in \mathscr{Y}_{l}^{r}} V^{\widehat{\mathfrak{s} l}(X)}(\bar{\lambda}) \otimes V^{\widehat{\mathfrak{s} l}(Y)}(\bar{t} \lambda) \otimes H_{|\lambda|}^{\widehat{a}} .
$$

We need to explain the notation: The set $\mathscr{Y}_{l}^{r}$ of generalized Young diagram consists of sequences $\lambda=\left(\lambda_{1}, \ldots, \lambda_{l}\right)\left(\lambda_{p} \in \mathbb{Z}, \lambda_{1} \geq \cdots \geq \lambda_{l}\right)$ with the level $r$ constraint $\lambda_{1}-\lambda_{l} \leq r$. The size of $\lambda$ is $|\lambda|=\sum_{p=1}^{l} \lambda_{i}$. We define the Maya diagram $M(\lambda)$ associated with $\lambda$ by

$$
m_{i p}(n)= \begin{cases}\square & \text { if } r\left(n-\frac{1}{2}\right)+i \leq \lambda_{p} \\ \square & \text { otherwise }\end{cases}
$$

A generalized Young diagram $\lambda$ defines a dominant weight of $\widehat{\mathfrak{s l}}(X)$ of level $r$ by

$$
\left(r-\lambda_{1}+\lambda_{l}\right) \Lambda_{0}+\sum_{p=1}^{l-1}\left(\lambda_{p}-\lambda_{p+1}\right) \Lambda_{p},
$$

where $\Lambda_{p}$ is the $p^{\text {th }}$ fundamental weight. We denote it by $\bar{\lambda}$. Then $\widehat{V^{\mathfrak{s l}}(X)}(\bar{\lambda})$ is the corresponding irreducible integrable highest weight module of $\widehat{\mathfrak{s l}}(X)$. Conversely a dominant weight $\bar{\lambda}$ with level $r$ gives a generalized Young diagram unique up to shift: $\left(\lambda_{1}, \ldots, \lambda_{l}\right) \mapsto\left(\lambda_{1}+k, \ldots, \lambda_{l}+k\right)$.

The transposition ${ }^{t}: \mathscr{Y}_{l}^{r} \rightarrow \mathscr{Y}_{r}^{l}$ is defined by $m_{p i}(n) \stackrel{\text { def }}{=} m_{i p}(n)$, i.e., the transposition of each $(l \times r)$ rectangle in the Maya diagram. Then ${ }^{t} \lambda$ defines a dominant weight ${ }^{\bar{t} \lambda}$ of $\widehat{\mathfrak{s l}}(Y)$ of level $l$. The corresponding representation is denoted by $\widehat{V^{\mathfrak{s l}}(Y)}\left(\overline{{ }^{t} \lambda}\right)$.

Finally $H_{k}^{\widehat{a}}$ denote the irreducible representation of $\widehat{a}$ with charge $k$. Here the charge is the eigenvalue of $J(0)$ which counts the number of $\square$ in the region $n>0$ minus the number of $\square$ in $n<0$.

The basis vector corresponding to the Maya diagram $M(\lambda)\left(\lambda \in \mathscr{Y}_{l}^{r}\right)$ gives the highest weight vector of $V^{\widehat{\mathfrak{s} r}(X)}(\bar{\lambda}) \otimes V^{\widehat{\mathfrak{s} l}(Y)}\left(\overline{{ }^{t} \lambda}\right) \otimes H_{|\lambda|}^{\widehat{a}}$ in the decomposition (A.2).

We have $\widehat{\mathfrak{s l}}(Y) \oplus \widehat{a} \cong \widehat{\mathfrak{g l} l}(Y)$. We denote $V^{\widehat{\mathfrak{s l} l}(Y)}(\bar{\lambda}) \otimes H_{|\lambda|}^{\widehat{a}}$ by $V^{\widehat{\mathfrak{g l} l}(Y)}\left({ }^{t} \lambda\right)$. Note that the ambiguity of the shift disappears if we consider ${ }^{t} \lambda$ as a weight for $\widehat{\mathfrak{g l}}(Y)$. Then (A.2) can be rewritten as

$$
F \cong \bigoplus_{\lambda \in \mathscr{Y}_{l}^{r}} V^{\widehat{\mathfrak{s} l}(X)}(\lambda) \otimes V^{\widehat{\mathfrak{g} l}(Y)}(\bar{t} \lambda)
$$

Let $\lambda \in \mathscr{Y}_{l}^{r}$ and $\bar{\mu}$ be a dominant weight of $\widehat{\mathfrak{s l}}(X)$ of level $r$. Then the weight space $V^{\widehat{\mathfrak{s l}}(X)}(\bar{\lambda})_{\bar{\mu}}$ is isomorphic to

$$
V^{\widehat{\mathfrak{s} l}(X)}(\bar{\lambda})_{\bar{\mu}} \cong \operatorname{Hom}_{\widehat{\mathfrak{g l}}(Y)_{l} \oplus \widehat{\mathfrak{h}}(\widehat{\mathfrak{s} l}(X))}\left(V^{\widehat{\mathfrak{g} l}(Y)}\left({ }^{t} \lambda\right) \otimes \mathbb{C}_{\bar{\mu}}, F\right)
$$




$$
\cong \bigoplus \operatorname{Hom}_{\widehat{\mathfrak{g l}}(Y)_{l} \oplus \widehat{\mathfrak{h}}(\widehat{\mathfrak{g} l}(X))}\left(V^{\widehat{\mathfrak{g} l}(Y)}\left({ }^{t} \lambda\right) \otimes \mathbb{C}_{\mu}, F\right)
$$

where $\widehat{\mathfrak{h}}(\widehat{\mathfrak{s l}}(X))$ (resp. $\widehat{\mathfrak{h}}(\widehat{\mathfrak{g l}}(X)))$ is the Cartan subalgebra of $\widehat{\mathfrak{s l}}(X)($ resp. $\widehat{\mathfrak{g l}}(X)), \mathbb{C}_{\bar{\mu}}$ (resp. $\left.\mathbb{C}_{\mu}\right)$ is its representation with weight $\bar{\mu}$ (resp. $\mu$ ), and $\bigoplus$ runs over all $\mu \in \mathscr{Y}_{l}^{r}$ whose corresponding $\widehat{\mathfrak{s l}}(X)$-weight is the given $\bar{\mu}$. According to $\mu$, the space $X$ decomposes into direct sum of 1 dimensional eigenspaces, and hence we have

$$
\operatorname{Hom}_{\widehat{\mathfrak{h}}(\widehat{\mathfrak{g} l}(X))}\left(\mathbb{C}_{\mu}, F\right) \cong \bigotimes_{p=1}^{l} V^{\widehat{\mathfrak{g} l}(Y)}\left(\Lambda_{\mu_{p}}\right)
$$

where $\Lambda_{\mu_{p}}$ is the $\mu_{p}^{\text {th }}$-fundamental weight of $\widehat{\mathfrak{s l}}(Y)$ with $\mu_{p}$ understood modulo $r$, and $V^{\widehat{\mathfrak{g} r}(Y)}\left(\Lambda_{\mu_{p}}\right)$ is $V^{\widehat{s}(Y)}\left(\Lambda_{\mu_{p}}\right) \otimes H_{\mu_{p}}^{\widehat{a}}$. Thus we get

$$
V^{\widehat{\mathfrak{s l}}(X)}(\bar{\lambda})_{\bar{\mu}} \cong \bigoplus \operatorname{Hom}_{\widehat{\mathfrak{g l}}(Y)_{l}}\left(V^{\widehat{\mathfrak{g} l}(Y)}\left({ }^{t} \lambda\right), \bigotimes_{p=1}^{l} V^{\widehat{\mathfrak{g} l}(Y)}\left(\Lambda_{\mu_{p}}\right)\right)
$$

However the right hand side is 0 unless $|\lambda|=|\mu|$, so we have at most one $\mu$ contributing to $\bigoplus$. On the other hand $(|\lambda| \bmod l)$ is well-defined for $\bar{\lambda}$. If $\bar{\mu}$ is a weight of $V^{\widehat{\mathfrak{s l}}(X)}(\bar{\lambda})$, then we must have $|\lambda| \equiv|\mu|(\bmod l)$. Hence we have exactly one $\mu$ with $|\lambda|=|\mu|$ and

$$
V^{\widehat{\mathfrak{s} l}(X)}(\bar{\lambda})_{\bar{\mu}} \cong \operatorname{Hom}_{\widehat{\mathfrak{g l} l}(Y)_{l}}\left(V^{\widehat{\mathfrak{g} l}(Y)}\left({ }^{t} \lambda\right), \bigotimes_{p=1}^{l} V^{\widehat{\mathfrak{g} l}(Y)}\left(\Lambda_{\mu_{p}}\right)\right)
$$

Now we incorporate the degree operator. Recall that an irreducible highest weight module $V^{\widehat{\mathfrak{s} l}(X)}(\bar{\lambda})$ of $\widehat{\mathfrak{s l}}(X)$ has a lift to $\mathfrak{s l}(X)_{\text {aff }}=\widehat{\mathfrak{s l}}(X) \oplus \mathbb{C} d^{\hat{\mathfrak{s} l}(X)}$, which is unique if we fix the value of $\left\langle d^{X}, \bar{\lambda}\right\rangle[12, \S 9.10]$. The same is true for $\widehat{\mathfrak{g l}}(Y)$. We fix $\left\langle d^{X}, \bar{\lambda}\right\rangle,\left\langle d^{Y},{ }^{t} \lambda\right\rangle$ (and hence the lifts of $\left.V^{\widehat{\mathfrak{s l}}(X)}(\bar{\lambda}), V^{\widehat{\mathfrak{g} l}(Y)}\left({ }^{t} \lambda\right)\right)$ so that we have

$$
d=d^{X} \otimes 1+1 \otimes d^{Y}
$$

in (A.3). This is possible since $d$ gives the degree operator for $\widehat{\mathfrak{s l}}(X)$ and $\widehat{\mathfrak{g l}}(Y)$. Therefore $\left\langle d^{X}, \bar{\lambda}\right\rangle+\left\langle d^{Y},{ }^{t} \lambda\right\rangle$ is equal to the eigenvalue of $d$ for the highest weight vector $v_{\bar{\lambda}} \otimes v_{t} \lambda$, i.e., the vector corresponding to the Maya diagram $M(\lambda)$. Let us denote the eigenvalue by $\langle d, M(\lambda)\rangle$. We also have $d$ on (A.4), the restriction from that on $F$. Thus $\bigotimes_{p=1}^{l} V^{\widehat{\mathfrak{g} l}(Y)}\left(\Lambda_{\mu_{p}}\right)$ has a natural

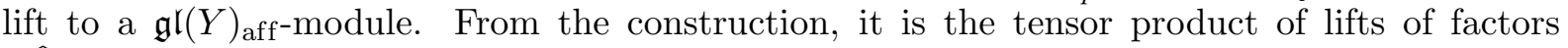
$V^{\widehat{\mathfrak{g} l}(Y)}\left(\Lambda_{\mu_{p}}\right)$ and the eigenvalue of $d$ for the highest weight vector $\otimes v_{\Lambda_{\mu_{p}}}$ is equal to that for the vector corresponding to the Maya diagram $M(\mu)$, i.e., $\langle d, M(\mu)\rangle$. Thus $\bigotimes_{p=1}^{l} V^{\widehat{\mathfrak{g} l}(Y)}\left(\Lambda_{\mu_{p}}\right)$ is

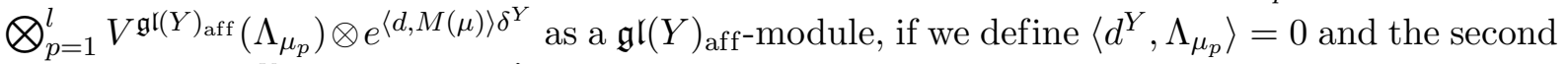
factor $e^{\langle d, M(\mu)\rangle \delta^{Y}}$ is the trivial $\widehat{\mathfrak{g l}}(Y)$-module with $d^{Y}$ acting by the multiplication by $\langle d, M(\mu)\rangle$. Then the right hand side of (A.5) has the induced operator $d$, which is equal to $d^{X}$ in the left hand side from the definition. Thus we can decompose both sides of (A.5) into eigenspaces of $d$ :

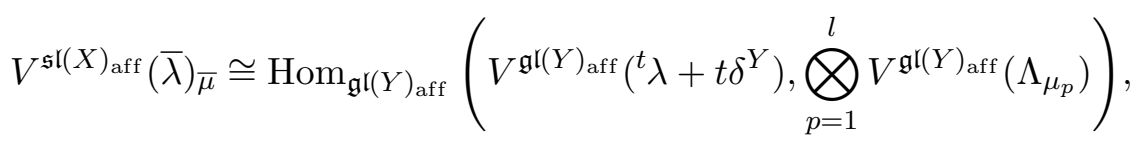

where we have chosen a lift of $\bar{\mu}$ to a weight of $\mathfrak{s l}(X)_{\text {aff }}$ and denoted it by the same notation, and $t=\left\langle d^{X}, \bar{\mu}\right\rangle-\langle d, M(\mu)\rangle$. We have the relation

$$
\left\langle d^{X}, \bar{\lambda}-\bar{\mu}\right\rangle=-\left\langle d^{Y},{ }^{t} \lambda+t \delta^{Y}\right\rangle+\langle d, M(\lambda)\rangle-\langle d, M(\mu)\rangle .
$$


The above weights are related to those in the main body of the paper (Remarks 5.11) by

$$
\mathbf{w}=\sum_{i} w_{i} \Lambda_{i}=\sum_{p=1}^{l} \Lambda_{\mu_{p}}, \quad \mathbf{w}-\mathbf{v}=\sum_{i} w_{i} \Lambda_{i}-v_{i} \alpha_{i}=\overline{{ }^{\dagger} \lambda}+t \delta^{Y} .
$$

\section{A.2 Tensor product multiplicities}

We decompose as $Y=Y_{1} \oplus Y_{2}$ with $\operatorname{dim} Y_{\alpha}=r_{\alpha}(\alpha=1,2)$. Then we have $\mathscr{L}(X \otimes Y) \cong$ $\mathscr{L}\left(X \otimes Y_{1}\right) \oplus \mathscr{L}\left(X \otimes Y_{2}\right)$ and hence

$$
\bigwedge^{\infty / 2} \mathscr{L}(X \otimes Y) \cong \bigwedge^{\infty / 2} \mathscr{L}\left(X \otimes Y_{1}\right) \otimes \bigwedge^{\infty / 2} \mathscr{L}\left(X \otimes Y_{2}\right)
$$

We apply the decomposition (A.3) to both hand sides:

$$
\bigoplus_{\lambda \in \mathscr{Y}_{l}^{r}} V^{\widehat{\mathfrak{s} l}(X)}(\bar{\lambda}) \otimes V^{\widehat{\mathfrak{g} l}(Y)}\left({ }^{t} \lambda\right) \cong \bigoplus_{\substack{\lambda_{1} \in \mathscr{Y}_{l}^{r_{1}} \\ \lambda_{2} \in \mathscr{Y}_{l}^{r_{2}}}} V^{\widehat{\mathfrak{s} l}(X)}\left(\bar{\lambda}_{1}\right) \otimes V^{\widehat{\mathfrak{s} l}(X)}\left(\bar{\lambda}_{2}\right) \otimes V^{\widehat{\mathfrak{g} l}\left(Y_{1}\right)}\left({ }^{t} \lambda_{1}\right) \otimes V^{\widehat{\mathfrak{g} l}\left(Y_{2}\right)}\left({ }^{t} \lambda_{2}\right)
$$

Hence

$$
\begin{aligned}
& \operatorname{Hom}_{\widehat{\mathfrak{s} l}(X)_{r}}\left(V^{\widehat{\mathfrak{s} l}(X)}(\bar{\lambda}), V^{\widehat{\mathfrak{s l}}(X)}\left(\bar{\lambda}_{1}\right) \otimes V^{\widehat{\mathfrak{s} l}(X)}\left(\bar{\lambda}_{2}\right)\right)
\end{aligned}
$$

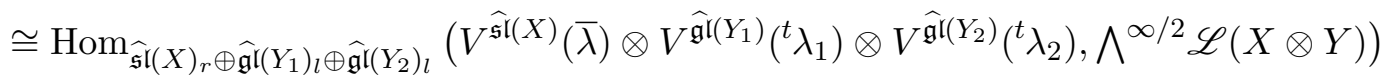

$$
\begin{aligned}
& \cong \bigoplus \operatorname{Hom}_{\widehat{\mathfrak{g l}}\left(Y_{1}\right)_{l} \oplus \widehat{\mathfrak{g} l}\left(Y_{2}\right)_{l}}\left(V^{\widehat{\mathfrak{g} l}\left(Y_{1}\right)}\left({ }^{t} \lambda_{1}\right) \otimes V^{\widehat{\mathfrak{g} l}\left(Y_{2}\right)}\left({ }^{t} \lambda_{2}\right), V^{\widehat{\mathfrak{g} l}(Y)}\left({ }^{t} \lambda\right)\right),
\end{aligned}
$$

where the summation runs over all $\lambda$ whose corresponding $\mathfrak{s l}(Y)$-weight is the given $\bar{\lambda}$. Since this is 0 unless $|\lambda|=\left|\lambda_{1}\right|+\left|\lambda_{2}\right|$, we only have a single summand as in the previous subsection, and hence

$$
\begin{aligned}
& \operatorname{Hom}_{\widehat{\mathfrak{s l} l}(X)_{r}}\left(V^{\widehat{\mathfrak{s} l}(X)}(\bar{\lambda}), V^{\widehat{\mathfrak{s} l}(X)}\left(\bar{\lambda}_{1}\right) \otimes V^{\widehat{\mathfrak{s l} l}(X)}\left(\bar{\lambda}_{2}\right)\right) \\
& \quad \cong \operatorname{Hom}_{\widehat{\mathfrak{g l} l}\left(Y_{1}\right)_{l} \oplus \widehat{\mathfrak{g} l}\left(Y_{2}\right)_{l}}\left(V^{\widehat{\mathfrak{g} l}\left(Y_{1}\right)}\left({ }^{t} \lambda_{1}\right) \otimes V^{\widehat{\mathfrak{g} l}\left(Y_{2}\right)}\left({ }^{t} \lambda_{2}\right), V^{\widehat{\mathfrak{g} l}(Y)}\left({ }^{t} \lambda\right)\right) .
\end{aligned}
$$

We can incorporate the degree operator after fixing the values of $\left\langle d^{X}, \bar{\lambda}\right\rangle,\left\langle d^{X}, \bar{\lambda}_{1}\right\rangle,\left\langle d^{X}, \bar{\lambda}_{2}\right\rangle$ as in the previous subsection. We have

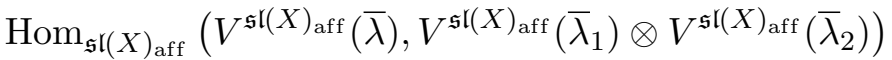

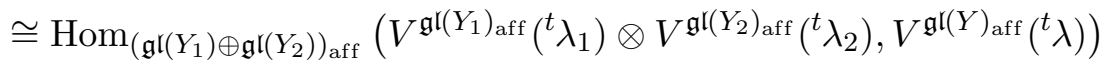

with the relation

$$
\begin{aligned}
& \left\langle d^{X}, \bar{\lambda}\right\rangle-\left\langle d^{X}, \bar{\lambda}_{1}\right\rangle-\left\langle d^{X}, \bar{\lambda}_{2}\right\rangle \\
& \quad=-\left\langle d^{Y},{ }^{t} \lambda\right\rangle+\left\langle d^{Y},{ }^{t} \lambda_{1}\right\rangle+\left\langle d^{Y},{ }^{t} \lambda_{2}\right\rangle+\langle d, M(\lambda)\rangle-\left\langle d, M\left(\lambda_{1}\right)\right\rangle-\left\langle d, M\left(\lambda_{2}\right)\right\rangle .
\end{aligned}
$$

We need to re-write this isomorphism in terms of $\mathfrak{s l}\left(Y_{1}\right)_{\text {aff }}, \mathfrak{s l}\left(Y_{2}\right)_{\text {aff }}$. Let $a_{1} \subset \mathfrak{g l}\left(Y_{1}\right)$, $a_{2} \subset \mathfrak{g r}\left(Y_{2}\right)$ be the central subalgebras generated by $\sum_{i=1}^{r_{1}} J_{i}^{i}(0)$ and $\sum_{i=r_{1}+1}^{r} J_{i}^{i}(0)$ respectively. Let $\widehat{a}_{1}, \widehat{a}_{2}$ be the corresponding Heisenberg subalgebras of $\widehat{\mathfrak{g l}}\left(Y_{1}\right), \widehat{\mathfrak{g l}}\left(Y_{2}\right)$. We consider the subalgebras

$$
\begin{aligned}
& a^{0} \stackrel{\text { def }}{=}\left\langle J^{0}(0) \stackrel{\text { def }}{=}-r_{2} \sum_{i=1}^{r_{1}} J_{i}^{i}(0)+r_{1} \sum_{j=r_{1}+1}^{r} J_{j}^{j}(0)\right\rangle \subset a_{1} \oplus a_{2}, \\
& \widehat{a}^{0} \stackrel{\text { def }}{=}\left\langle J^{0}(n) \stackrel{\text { def }}{=}-r_{2} \sum_{i=1}^{r_{1}} J_{i}^{i}(n)+r_{1} \sum_{j=r_{1}+1}^{r} J_{j}^{j}(n)\right\rangle_{n \in \mathbb{Z}} \subset \widehat{a}_{1} \oplus \widehat{a}_{2} .
\end{aligned}
$$


We also put $a_{\mathrm{aff}}^{0} \stackrel{\text { def }}{=} \widehat{a}^{0} \oplus \mathbb{C} d$ with the usual commutator relation. We have $a_{1} \oplus a_{2}=a^{0} \oplus a$, where $a$ is the subalgebra generated by $J(0)$. Therefore

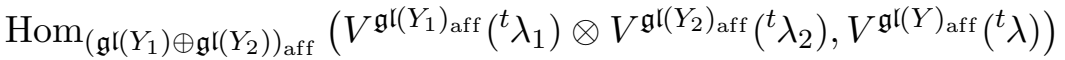

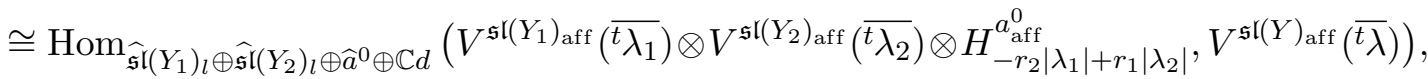

where the charge of $a_{\mathrm{aff}}^{0}$ is defined as the eigenvalue of $J^{0}(0)$ and the eigenvalue of $d$ for the vacuum vector of $H_{-r_{2}\left|\lambda_{1}\right|+r_{1}\left|\lambda_{2}\right|}^{a_{\text {aff }}^{0}}$ is set to be 0 . This space is isomorphic to the space of vectors in

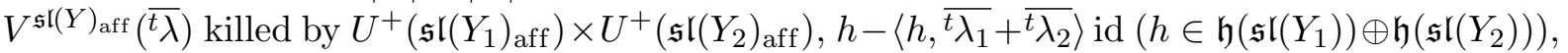
$J^{0}(n)-\delta_{0 n}\left(-r_{2}\left|\lambda_{1}\right|+r_{1}\left|\lambda_{2}\right|\right)(n \geq 0)$ and $d-\left\langle d^{Y},{ }^{t} \lambda_{1}\right\rangle-\left\langle d^{Y},{ }^{t} \lambda_{2}\right\rangle$ by assigning the image of the tensor product of the highest weight vectors and the vacuum vector of a homomorphism.

Consider

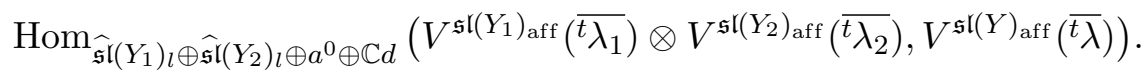

This is isomorphic to the space of vectors as above, except that the condition for $J^{0}(n)$ is required only for $n=0$. This space is an $\widehat{a}^{0}$-module, and is spanned by vectors obtained from vectors in (A.8) applying various $J^{0}(n)$ for $n<0$. But since $\left[d, J^{0}(n)\right]=n J^{0}(n)$, the condition for $d$ also implies that they must lie in (A.8). We get

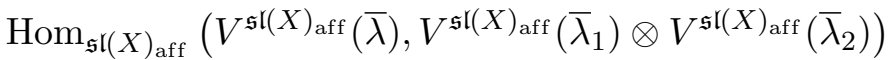

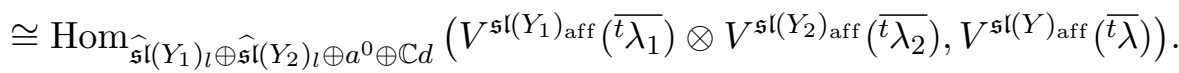

\section{Acknowledgments}

This work is supported by the Grant-in-aid for Scientific Research (No.19340006), JSPS. This work was started while the author was visiting the Institute for Advanced Study with supports by the Ministry of Education, Japan and the Friends of the Institute. The author would like to thank to A. Braverman and M. Finkelberg for discussion on the subject, and to the referees for their careful readings and comments.

\section{References}

[1] Bando S., Einstein-Hermitian metrics on noncompact K ähler manifolds, in Einstein Metrics and YangMills connections (Sanda, 1990), Lecture Notes in Pure and Appl. Math., Vol. 145, Dekker, New York, 1993, $27-33$.

[2] Baranovsky V., Moduli of sheaves on surfaces and action of the oscillator algebra, J. Differential Geom. 55 (2000), 193-227, math.AG/9811092.

[3] Borho W., MacPherson R., Partial resolutions of nilpotent varieties, in Analysis and Topology on Singular Spaces, II, III (Luminy, 1981), Astérisque, Vol. 101, Soc. Math. France, Paris, 1983, $23-74$.

[4] Braverman A., Finkelberg M., Pursuing the double affine Grassmannian I: transversal slices via instantons on $A_{k}$-singularities, arXiv:0711.2083.

[5] Braverman A., Finkelberg M., Private communication, 2008.

[6] Braverman A., Kazhdan D., The spherical Hecke algebra for affine Kac-Moody groups I, arXiv:0809.1461.

[7] Chriss N., Ginzburg V., Representation theory and complex geometry, Birkhäuser Boston Inc., Boston, MA, 1997.

[8] Crawley-Boevey W., Geometry of the moment map for representations of quivers, Compositio Math. 126 (2001), 257-293.

[9] Frenkel I.B., Representations of affine Lie algebras, Hecke modular forms and Korteweg-de Vries type equations, in Lie Algebras and Related Topics (New Brunswick, N.J., 1981), Lecture Notes in Math., Vol. 933, Springer, Berlin - New York, 1982, 71-110. 
[10] Grojnowski I., Instantons and affine algebras. I. The Hilbert scheme and vertex operators, Math. Res. Lett. 3 (1996), 275-291, alg-geom/9506020.

[11] Hasegawa K., Spin module versions of Weyl's reciprocity theorem for classical Kac-Moody Lie algebras an application to branching rule duality, Publ. Res. Inst. Math. Sci. 25 (1989), 741-828.

[12] Kac V.G., Infinite-dimensional Lie algebras, 3rd ed., Cambridge University Press, Cambridge, 1990.

[13] Kashiwara M., Saito Y., Geometric construction of crystal bases, Duke Math. J. 89 (1997), 9-36, q-alg/9606009.

[14] King A.D., Moduli of representations of finite-dimensional algebras, Quart. J. Math. Oxford Ser. (2) 45 (1994), 515-530.

[15] Kronheimer P.B., The construction of ALE spaces as hyper-Kähler quotients, J. Differential Geom. 29 (1989), 665-683.

[16] Kronheimer P.B., Nakajima H., Yang-Mills instantons on ALE gravitational instantons, Math. Ann. 288 (1990), 263-307.

[17] Lusztig G., Green polynomials and singularities of unipotent classes, Adv. in Math. 42 (1981), 169-178.

[18] Lusztig G., Canonical bases arising from quantized enveloping algebras. II, in Common Trends in Mathematics and Quantum Field Theories (Kyoto, 1990), Progr. Theoret. Phys. Suppl. (1990), no. 102, 175-201.

[19] Malkin A., Tensor product varieties and crystals: the ADE case, Duke Math. J. 116 (2003), 477-524, math.AG/0103025.

[20] Mirković I., Vilonen K., Perverse sheaves on affine Grassmannians and Langlands duality, Math. Res. Lett. 7 (2000), 13-24, math.AG/9911050.

[21] Nagao K., Quiver varieties and Frenkel-Kac construction, math.RT/0703107.

[22] Nakajima H., Instantons on ALE spaces, quiver varieties, and Kac-Moody algebras, Duke Math. J. 76 (1994), 365-416.

[23] Nakajima H., Varieties associated with quivers, in Representation Theory of Algebras and Related Topics (Mexico City, 1994), CMS Conf. Proc., Vol. 19, Amer. Math. Soc., Providence, RI, 1996, 139-157.

[24] Nakajima H., Quiver varieties and Kac-Moody algebras, Duke Math. J. 91 (1998), 515-560.

[25] Nakajima H., Lectures on Hilbert schemes of points on surfaces, University Lecture Series, Vol. 18, American Mathematical Society, Providence, RI, 1999.

[26] Nakajima H., Quiver varieties and finite-dimensional representations of quantum affine algebras, J. Amer. Math. Soc. 14 (2001), 145-238, math.QA/9912158.

[27] Nakajima H., Quiver varieties and tensor products, Invent. Math. 146 (2001), 399-449, math.QA/0103008.

[28] Nakajima H., Geometric construction of representations of affine algebras, in Proceedings of the International Congress of Mathematicians, Vol. I (Beijing, 2002), Higher Ed. Press, Beijing, 2002, 423-438, math.QA/0212401.

[29] Nakajima H., Reflection functors for quiver varieties and Weyl group actions, Math. Ann. 327 (2003), 671-721.

[30] Nakajima H., Sheaves on ALE spaces and quiver varieties, Mosc. Math. J. 7 (2007), 699-722.

[31] Nakanishi T., Tsuchiya A., Level-rank duality of WZW models in conformal field theory, Comm. Math. Phys. 144 (1992), 351-372.

[32] Rudakov A., Stability for an abelian category, J. Algebra 197 (1997), 231-245.

[33] Saito Y., Crystal bases and quiver varieties, Math. Ann. 324 (2002), 675-688, math.QA/0111232. 